\title{
Diophantine approximation on rational quadrics
}

\section{Cornelia Druţu}

Received: 17 December 2003 / Revised version: 4 April 2005 /

Published online: 20 July 2005 - (C) Springer-Verlag 2005

\begin{abstract}
We compute the Hausdorff dimension of sets of very well approximable vectors on rational quadrics. We use ubiquitous systems and the geometry of locally symmetric spaces. As a byproduct we obtain the Hausdorff dimension of the set of rays with a fixed maximal singular direction, which move away into one end of a locally symmetric space at linear depth, infinitely many times.
\end{abstract}

Mathematics Subject Classification (2000): 11J83, 22E40, 53C35.

\section{Contents}

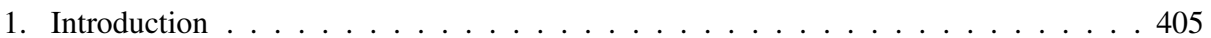

2. Preliminaries on (locally) symmetric spaces . . . . . . . . . . . . . . 415

3. Symmetric spaces of positive definite quadratic forms . . . . . . . . . . . . . . . 435

4. Diophantine approximation on a rational quadric . . . . . . . . . . . . . . . . . . . . . . . . . . . .

5. Sets of geodesic rays moving away into the cusp . . . . . . . . . . . . . . 458

\section{Introduction}

The main result in the present paper is the computation of the Hausdorff dimensions of the sets of very well approximable vectors on a rational quadric $\mathfrak{Q}$. The method is to consider the rational non-degenerate quadratic form $\mathfrak{q}: \mathbb{R}^{n} \rightarrow \mathbb{R}$ such that the quadric $\mathfrak{Q}$ is defined by $\mathfrak{q}=1$ and the quadratic form $L_{\mathfrak{q}}: \mathbb{R}^{n+1} \rightarrow$ $\mathbb{R}, L_{\mathfrak{q}}\left(x_{1}, \ldots, x_{n+1}\right)=x_{n+1}^{2}-\mathfrak{q}\left(x_{1}, \ldots, x_{n}\right)$. The connected component of the identity $S O_{I}\left(L_{\mathfrak{q}}\right)$ of the stabilizer $S O\left(L_{\mathfrak{q}}\right)$ of the form $L_{\mathfrak{q}}$ is a semisimple group (simple if $n \neq 3$ ). The integer points of this group compose a lattice. One can consider the symmetric space associated to $S O_{I}\left(L_{\mathfrak{q}}\right)$ and its quotient by the lattice, which is a locally symmetric space. The set of very well approximable vectors on $\mathfrak{Q}$ can be defined in terms of the geometry of the locally symmetric space, and its Hausdorff dimension can be estimated using an ubiquitous system which appears in this context and the general properties of ubiquitous systems.

C. DRUŢU

UFR de Mathématiques et UMR CNRS no. 8524, Université de Lille I, F-59655 Villeneuve d'Ascq, France

(e-mail: Cornelia.Drutu@math.univ-lillel.fr) 


\subsection{Hausdorff dimension of sets of very well approximable vectors in $\mathbb{R}^{n}$}

We denote by $\|\cdot\|_{e}$ the Euclidean norm and by $\|\cdot\|$ the max-norm in $\mathbb{R}^{n}$,

$$
\|x\|=\max \left\{\left|x_{1}\right|,\left|x_{2}\right|, \ldots,\left|x_{n}\right|\right\} .
$$

Throughout $\psi: \mathbb{R}_{+} \rightarrow \mathbb{R}_{+}$denotes a decreasing function satisfying $\lim _{x \rightarrow \infty} \psi(x)=0$, also called an approximating function. Rational vectors are always written in the form $\frac{1}{q} \bar{p}$, where $q \in \mathbb{N}, \bar{p}=\left(p_{1}, \ldots, p_{n}\right) \in \mathbb{Z}^{n}$ and $\operatorname{gcd}\left(q, p_{1}, \ldots, p_{n}\right)=1$.

Let $M$ be a submanifold of $\mathbb{R}^{n}$. The set of simultaneously $\psi$-approximable vectors in $M$ is defined by

$\mathcal{S}_{\psi}(M)=\left\{\bar{x} \in M ;\|q \bar{x}-\bar{p}\| \leq \psi(q)\right.$ for infinitely many $\left.q \in \mathbb{N}, \bar{p} \in \mathbb{Z}^{n}\right\}$.

In the particular case when $\psi(x)=\frac{1}{x^{\alpha}}$ with $\alpha>\frac{1}{n}$, the set is also denoted by $\mathcal{S}_{\alpha}(M)$ and it is called the set of simultaneously $\alpha$-very well approximable vectors in $M$. A subset of it is the set of simultaneously exactly- $\alpha$-very well approximable vectors in $M$,

$$
\mathcal{E S}_{\alpha}(M)=\left\{\bar{x} \in M ; \bar{x} \in \mathcal{S}_{\alpha}(M) \text { and } \bar{x} \notin \mathcal{S}_{\beta}(M), \forall \beta>\alpha\right\} .
$$

Likewise is defined the set of linearly $\psi$-approximable vectors in $M$, by $\mathcal{L}_{\psi}(M)=\left\{\bar{x} \in M ;|\bar{q} \cdot \bar{x}-p| \leq \psi(\|\bar{q}\|)\right.$ for infinitely many $\left.\bar{q} \in \mathbb{Z}^{n}, p \in \mathbb{N}\right\}$, where $\bar{q} \cdot \bar{x}=\sum_{i=1}^{n} q_{i} x_{i}$. In particular when $\psi(x)=x^{-\beta}$, with $\beta>n$, the previous set is denoted by $\mathcal{L}_{\beta}(M)$ and it is called the set of linearly $\beta$-very well approximable vectors in $M$.

Khintchine's transference principle [BD, §1.3.1] implies that

$$
\bigcup_{\alpha>1 / n} \mathcal{S}_{\alpha}\left(\mathbb{R}^{n}\right)=\bigcup_{\beta>n} \mathcal{L}_{\beta}\left(\mathbb{R}^{n}\right) .
$$

The vectors in this set are called very well approximable vectors. A consequence of the Khintchine-Groshev Theorem [BD, §1.3.4] is that the set of very well approximable vectors in $\mathbb{R}^{n}$ is of Lebesgue measure zero. Thus, in order to study the sets of type $\mathcal{S}_{\psi}$ and $\mathcal{L}_{\psi}$ when $\psi$ decreases sufficiently quickly at infinity so that they are of Lebesgue measure zero, a more appropriate tool is the Hausdorff dimension and the Hausdorff measure. In the sequel we denote by $\operatorname{dim}_{H} A$ the Hausdorff dimension of a subset $A$ in a metric space. We denote by $\mathcal{H}^{s}$ the Hausdorff measure corresponding to the parameter $s$ (see Section 4.2 for definitions). It has been proved in [Ja] that given $s \in[0, n)$,

$$
\mathcal{H}^{s}\left(\mathcal{S}_{\psi}\left(\mathbb{R}^{n}\right)\right)= \begin{cases}0, & \text { if } \sum_{k=1}^{\infty} k^{n-s} \psi(k)^{s}<\infty \\ \infty, & \text { if } \sum_{k=1}^{\infty} k^{n-s} \psi(k)^{s}=\infty\end{cases}
$$


In particular, for any $\alpha>\frac{1}{n}$

$$
d=\operatorname{dim}_{H} \mathcal{S}_{\alpha}\left(\mathbb{R}^{n}\right)=\frac{n+1}{\alpha+1} \text { and } \mathcal{H}^{d}\left(\mathcal{S}_{\alpha}\left(\mathbb{R}^{n}\right)\right)=\infty .
$$

This implies that both relations also hold for $\mathcal{E} \mathcal{S}_{\alpha}\left(\mathbb{R}^{n}\right)$ instead of $\mathcal{S}_{\alpha}\left(\mathbb{R}^{n}\right)$.

In $[\mathrm{BoD}]$ it was shown that

$$
\operatorname{dim}_{H} \mathcal{L}_{\beta}\left(\mathbb{R}^{n}\right)=n-1+\frac{n+1}{\beta+1}, \quad \forall \beta>n .
$$

Moreover the following holds [DV]. Let $s \in(n-1, n)$ and let $\psi$ be an approximating function. Then

$$
\mathcal{H}^{s}\left(\mathcal{L}_{\psi}\left(\mathbb{R}^{n}\right)\right)= \begin{cases}0, & \text { if } \sum_{k=1}^{\infty} k^{2 n-1-s} \psi(k)^{s-(n-1)}<\infty \\ \infty, & \text { if } \sum_{k=1}^{\infty} k^{2 n-1-s} \psi(k)^{s-(n-1)}=\infty\end{cases}
$$

\subsection{Known results on very well approximable vectors on manifolds}

The general question to ask is under what conditions the vectors in a submanifold $M$ of $\mathbb{R}^{n}$ behave similarly to the vectors in $\mathbb{R}^{n}$, with respect to Diophantine approximation. If $M$ is a rational affine subspace of dimension $k<n$ in $\mathbb{R}^{n}$ then $M=\mathcal{S}_{1}(M)$. Therefore, rational affine subspaces must be avoided.

M.M. Dodson, B.P. Rynne and J.A.G. Vickers have shown in $\left[\mathrm{DRV}_{3}\right]$ and in $\left[\mathrm{DRV}_{4}\right]$ that under some non-zero curvature condition, the set of very well approximable vectors in $M$ is of measure 0. D. Kleinbock and G.A. Margulis have shown in $\left[\mathrm{KM}_{2}\right]$ the same result in a submanifold $M$ of $\mathbb{R}^{n}$ non-degenerate almost everywhere (they have actually shown that a larger set, the set of very well multiplicatively approximable vectors in $M$, has measure 0 in this case). A point $\bar{x} \in M$ is non-degenerate if in a neighborhood of $\bar{x}, M$ is not near to any affine subspace. More precisely, in a neighborhood of $\bar{x}$ the submanifold $M$ is parameterized by a function $f$ which is $l$ times continuously differentiable and such that its partial derivatives in $\bar{x}$ up to order $l$ span $\mathbb{R}^{n}$. A submanifold $M$ non-degenerate almost everywhere is a submanifold in which almost every point is non-degenerate.

A Khintchine-Groshev type theory was equally developed in the setting of manifolds. Concerning the linear approximation (that is, the Groshev type theory) it has been shown that any submanifold non-degenerate almost everywhere is of Groshev type (see [Ber], [BKM], [BBKM], [BDV 2$]$ ). For the known results in simultaneous approximation, that is for a Khintchine type theory on manifolds, we refer to $[\mathrm{BD}],\left[\mathrm{BDV}_{1}\right],\left[\mathrm{BDV}_{2}\right],\left[\mathrm{DRV}_{3}\right],\left[\mathrm{DRV}_{4}\right]$ and references therein.

Consider a submanifold $M$ with the set of very well approximable vectors of measure zero. Such a submanifold is also called extremal. One can ask what is the Hausdorff dimension of each set $\mathcal{S}_{\alpha}(M)$ with $\alpha>\frac{1}{n}$ and $\mathcal{L}_{\beta}(M)$ with $\beta>n$. More is known about the sets $\mathcal{L}_{\beta}(M)$. R.C. Baker [Bak] proved that if $M$ is a 
planar curve of class $C^{3}$ whose curvature is zero at most in a set of points of Hausdorff dimension zero, then

$$
\operatorname{dim}_{H} \mathcal{L}_{\beta}(M)=\frac{3}{1+\beta}, \forall \beta \geq 2 .
$$

M.M. Dodson, B.P. Rynne and J.A.G. Vickers $\left[\mathrm{DRV}_{1}\right]$ later proved that if $M$ is a $C^{3}$-submanifold of dimension $m \geq 2$ in $\mathbb{R}^{n}$ such that at least two principal curvatures are not zero except on a set of Hausdorff dimension at most $m-1$, then

$$
\operatorname{dim}_{H} \mathcal{L}_{\beta}(M)=m-1+\frac{n+1}{1+\beta}, \forall \beta \geq n .
$$

H. Dickinson and M.M. Dodson have shown in $\left[\mathrm{DD}_{2}\right]$ that if $M$ is extremal then

$$
\operatorname{dim}_{H} \mathcal{L}_{\beta}(M) \geq m-1+\frac{n+1}{1+\beta}, \forall \beta \geq n .
$$

Finally, in $\left[\mathrm{BDV}_{2}\right]$, V. Beresnevich, D. Dickinson and S. Velani have shown that, given $M$ an $m$-dimensional submanifold in $\mathbb{R}^{n}$, with $n \geq 2, M$ non-degenerate almost everywhere, the following holds. Consider $s \in(m-1, m)$. If

$$
\sum_{k=1}^{\infty} \psi(k)^{s-(m-1)} k^{n+m-1-s}=\infty \text { then } \mathcal{H}^{s}\left(\mathcal{L}_{\psi}(M)\right)=\infty .
$$

In particular for $\psi(x)=x^{-\beta}$ this implies the result of H. Dickinson and M.M. Dodson, under the given hypotheses for $M$, and moreover it shows that for $d=m-1+\frac{n+1}{1+\beta}$, the Hausdorff measure $\mathcal{H}^{d}\left(\mathcal{L}_{\beta}(M)\right)$ is $\infty$.

These results and Khintchine's transference principle can be used to obtain upper and lower bounds for the Hausdorff dimensions of the sets $\mathcal{S}_{\alpha}(M)$. As far as the exact Hausdorff dimension for sets $\mathcal{S}_{\alpha}$ goes, the known results are the following. In $\left[\mathrm{BDV}_{2}\right]$ it is shown that, given $\psi$ an approximating function such that $\lim _{x \rightarrow \infty} x \psi(x)=0$ and $s \in(0,1)$, the following holds.

$$
\mathcal{H}^{s}\left(\mathcal{S}_{\psi}\left(\mathbb{S}^{1}\right)\right)= \begin{cases}0, & \text { if } \sum_{k=1}^{\infty}\left(\frac{\psi(k)}{k}\right)^{s}<\infty, \\ \infty, & \text { if } \sum_{k=1}^{\infty}\left(\frac{\psi(k)}{k}\right)^{s}=\infty .\end{cases}
$$

In particular this implies that

$$
\operatorname{dim}_{H} \mathcal{S}_{\alpha}\left(\mathbb{S}^{1}\right)=\frac{1}{1+\alpha} \text { and } \mathcal{H}^{1 /(1+\alpha)}\left(\mathcal{S}_{\alpha}\left(\mathbb{S}^{1}\right)\right)=\infty, \forall \alpha>1
$$

The first equality in (7) had already been proved in $\left[\mathrm{DD}_{1}\right]$.

In [BD, Theorem 4.8] it is proved that if $k \in \mathbb{N}, k \geq 3$, and

$$
\mathcal{C}_{k}=\left\{(x, y) \in \mathbb{R}^{2} ; x^{k}+y^{k}=1\right\},
$$


then $\mathcal{S}_{\alpha}\left(\mathcal{C}_{k}\right)$ contains at most four points for $\alpha>k-1$, hence $\operatorname{dim}_{H} \mathcal{S}_{\alpha}\left(\mathcal{C}_{k}\right)=0$ for $\alpha>k-1$.

The examples of $\mathbb{S}^{1}$ and $\mathcal{C}_{k}, k \geq 3$, already emphasize that, unlike in the case of linear approximation, a condition of non-zero curvature is not enough to deduce the Hausdorff dimensions of the sets $\mathcal{S}_{\alpha}$. B.P. Rynne [Ry] showed moreover that for every $C^{k}$-submanifold $M$ of $\mathbb{R}^{n}$ of dimension $m$ there exist $C^{k}$-submanifolds $M_{z}$ and $M_{p}$ arbitrarily $C^{k}$-close to $M$ (in a suitable sense) such that for $\alpha$ sufficiently large $\mathcal{S}_{\alpha}\left(M_{z}\right)=\emptyset$ and $\operatorname{dim}_{H} \mathcal{S}_{\alpha}\left(M_{p}\right)>\frac{m+1}{k(\alpha+1)}$. It follows that conditions taking into account only the structure of differential submanifold and depending continuously on this structure cannot suffice to obtain information about $\mathcal{S}_{\alpha}(M)$, at least not for large values of $\alpha$. The following result from $\left[\mathrm{BDV}_{1}\right]$ on the other hand seems to indicate that for values of $\alpha$ near to $\frac{1}{n}$, where $n$ is the dimension of the ambient space $\mathbb{R}^{n}$, there should exists however a formula holding for any non-degenerate submanifold of $\mathbb{R}^{n}$. More precisely, in $\left[\mathrm{BDV}_{1}\right]$ it is shown the following. Let $f \in C^{3}([a, b]), a<b$, let $\mathcal{C}_{f}=\{(t, f(t)) ; t \in[a, b]\}$, let $s \in(1 / 2,1)$ and let $\psi$ be an approximating function.

- If $\sum_{k=1}^{\infty} k^{1-s} \psi(k)^{s+1}=\infty$ then $\mathcal{H}^{s}\left(\mathcal{S}_{\psi}\left(\mathcal{C}_{f}\right)\right)=\infty$;

- Let $\lambda_{\psi}=\liminf _{x \rightarrow \infty} \frac{-\ln \psi(x)}{\ln x}$. If the Hausdorff dimension of the set $\{t \in$ $\left.[a, b] ; f^{\prime \prime}(t)=0\right\}$ is at most $\frac{2-\lambda_{\psi}}{1+\lambda_{\psi}}$ then $d=\operatorname{dim}_{H}\left(\mathcal{S}_{\psi}\left(\mathcal{C}_{f}\right)\right)=\frac{2-\lambda_{\psi}}{1+\lambda_{\psi}}$. Assume moreover that $\lambda_{\psi} \in(1 / 2,1)$. Then $\lim _{\sup } \operatorname{su}_{\infty} x^{2-d} \psi(x)^{d+1}>0$ implies that $\mathcal{H}^{d}\left(\mathcal{S}_{\psi}\left(\mathcal{C}_{f}\right)\right)=\infty$.

In the particular case when $\psi(x)=x^{-\alpha}$ with $\alpha \in(1 / 2,1)$ this gives the following.

- $\operatorname{dim}_{H} \mathcal{S}_{\alpha}\left(\mathcal{C}_{f}\right) \geq d=\frac{2-\alpha}{1+\alpha}$ and $\mathcal{H}^{d}\left(\mathcal{S}_{\alpha}\left(\mathcal{C}_{f}\right)\right)=\infty$;

- If moreover the Hausdorff dimension of the set $\left\{t \in[a, b] ; f^{\prime \prime}(t)=0\right\}$ is at most $\frac{2-\alpha}{1+\alpha}$ then $\operatorname{dim}_{H} \mathcal{S}_{\alpha}\left(\mathcal{C}_{f}\right)=d$.

In the particular case of a rational quadric $\mathfrak{Q}$ in $\mathbb{R}^{2}$ one obtains $\operatorname{dim}_{H} \mathcal{S}_{\alpha}(\mathfrak{Q})=$ $\frac{2-\alpha}{1+\alpha}$ for $\alpha \in[1 / 2,1)$. Note that for $\mathfrak{Q}=\mathbb{S}^{1}$ this differs from the formula for $\alpha>1$ given in (7). Thus in this case, unlike in the cases treated in (2), (3), (5) and (6), the Hausdorff dimension of the sets of very well approximable vectors is not a rational function in $\alpha$ but a piecewise rational function in $\alpha$, with different expressions for $\alpha \in[1 / 2,1)$ and for $\alpha>1$.

In [DL] the Hausdorff dimension of $\mathcal{S}_{\alpha}(M)$ has been computed for large values of $\alpha$ and for $M$ a manifold parameterized by polynomials with integer coefficients.

\subsection{Very well approximable vectors on rational quadrics}

The purpose of the present paper is to compute the Hausdorff dimension of the sets $\mathcal{S}_{\alpha}\left(\mathfrak{Q}_{\mathfrak{q}}\right)$ for $\alpha>1$, where $\mathfrak{Q}_{\mathfrak{q}}$ is a quadric defined by the equation $\mathfrak{q}=1$, for a given non-degenerate rational quadratic form $\mathfrak{q}: \mathbb{R}^{n} \rightarrow \mathbb{R}$. Obviously $\mathfrak{q}$ 
cannot be negative definite. The main result of the paper, formulated not in the most general form, is the following.

Theorem 1.1. Let $\psi$ be an approximating function such that $\lim _{x \rightarrow \infty} x \psi(x)=0$.

(1) If $\mathfrak{Q}_{\mathfrak{q}} \cap \mathbb{Q}^{n}=\emptyset$ then $\mathcal{S}_{\psi}\left(\mathfrak{Q}_{\mathfrak{q}}\right)=\varnothing$.

(2) If $\mathfrak{Q}_{\mathfrak{q}} \cap \mathbb{Q}^{n} \neq \emptyset$ then

$$
\operatorname{dim}_{H} \mathcal{S}_{\psi}\left(\mathfrak{Q}_{\mathfrak{q}}\right)=\sigma(n-1),
$$

where $\sigma=\limsup _{x \rightarrow \infty} \frac{\ln x}{\ln x-\ln \psi(x)}$.

Moreover, if $\lim \sup _{x \rightarrow \infty} x^{1-\sigma} \psi(x)^{\sigma}>0$ then $\mathcal{H}^{\sigma(n-1)}\left(\mathcal{S}_{\psi}\left(\mathfrak{Q}_{\mathfrak{q}}\right)\right)=\infty$.

In particular the set $\mathcal{S}_{\alpha}\left(\mathfrak{Q}_{\mathfrak{q}}\right)$ has Hausdorff dimension $d=\frac{n-1}{1+\alpha}$ for any $\alpha>1$

and $\mathcal{H}^{d}\left(\mathcal{S}_{\alpha}\left(\mathfrak{Q}_{\mathfrak{q}}\right)\right)=\infty$. Both statements also hold for the set $\mathcal{E} \mathcal{S}_{\alpha}\left(\mathfrak{Q}_{\mathfrak{q}}\right)$.

According to [BSh, Chapter 1, §7] a rational non-degenerate quadratic form in $n \geq 5$ variables takes the zero value on $\mathbb{Z}^{n} \backslash\{(0, \ldots, 0)\}$ if and only if it is not defined. This theorem applied to the form $L_{\mathfrak{q}}$ implies that for $n \geq 4, \mathfrak{Q}_{\mathfrak{q}} \cap \mathbb{Q}^{n} \neq \emptyset$ for any rational quadratic form $\mathfrak{q}$. For $n=2,3$ see [BSh, Chapter 1, §7].

\section{Outline of proof of Theorem 1.1.}

Statement (1) is a straightforward consequence of Lemma 4.1.1. Therefore we may assume that $\mathfrak{Q}_{\mathfrak{q}} \cap \mathbb{Q}^{n} \neq \emptyset$. The symmetric space corresponding to the semisimple group $S O_{I}\left(L_{\mathfrak{q}}\right)$ is the space $\mathcal{P}_{n+1}\left(L_{\mathfrak{q}}\right)$ of minimal positive definite quadratic forms $Q$ such that $\left|L_{\mathfrak{q}}(\bar{x})\right| \leq Q(\bar{x}), \forall \bar{x}$ (see [Bo] or Section 3.3). The boundary at infinity of it, $\partial_{\infty} \mathcal{P}_{n+1}\left(L_{\mathfrak{q}}\right)$, is a spherical building which can be canonically identified with the spherical building of flags of $\mathbb{R}^{n+1}$ composed of subspaces totally isotropic with respect to $L_{\mathfrak{q}}$ ([Mo, §15, §16], [Wi, §4.G]). In particular $\partial_{\infty} \mathcal{P}_{n+1}\left(L_{\mathfrak{q}}\right)$ contains a maximal singular stratum corresponding to the 1-dimensional subspaces totally isotropic with respect to $L_{\mathfrak{q}}$. We call it the stratum $\wp$ and the points composing it points of type $\wp$. Correspondingly we say that a geodesic ray in $\mathcal{P}_{n+1}\left(L_{\mathfrak{q}}\right)$ is of type $\wp$ if its point at infinity is.

Convention: Throughout, a semisimple group acts by isometries on the right on the symmetric space associated to it and on its boundary at infinity.

The quadric $\mathfrak{Q}_{\mathfrak{q}}$ can be identified with an open Zariski dense subset of the stratum $\wp$ in $\partial_{\infty} \mathcal{P}_{n+1}\left(L_{\mathfrak{q}}\right)$. On the other hand, for any geodesic ray $\varrho$ in $\mathcal{P}_{n+1}\left(L_{\mathfrak{q}}\right)$ of type $\wp$, the opposite unipotent $U_{+}(\varrho)$ of $\varrho$ (see Section 2.3 for a definition) can be identified with an open Zariski dense subset of the stratum $\wp$ via the bijection $\mathbf{u} \mapsto \varrho(\infty) \mathbf{u}$. With a countable covering argument we can replace in our study $\mathcal{S}_{\psi}\left(\mathfrak{Q}_{\mathfrak{q}}\right)$ by $\mathcal{S}_{\psi}(\Omega)$, where $\Omega$ is a relatively compact open subset whose closure is contained in the image of $U_{+}(\varrho)$ for some $\varrho$. The set $\Omega$ can be identified with a relatively compact open subset of $U_{+}(\varrho)$. 
Let $\Gamma=S O_{I}\left(L_{\mathfrak{q}}\right) \cap S L(n+1, \mathbb{Z})$. The locally symmetric space $\mathcal{V}=$ $\mathcal{P}_{n+1}\left(L_{\mathfrak{q}}\right) / \Gamma$ has ends if and only if $\mathfrak{Q}_{\mathfrak{q}} \cap \mathbb{Q}^{n} \neq \emptyset$. Moreover there exist finitely many geodesic rays $\bar{r}_{i}, i \in\{1,2, \ldots, k\}$, in $\mathcal{V}$ such that their lifts $r_{i}$ in $\mathcal{P}_{n+1}\left(L_{\mathfrak{q}}\right)$ are of type $\wp$ and the following holds. Let $r_{i} \Gamma$ be the $\Gamma$-orbit of $r_{i}$, let $C s p_{i}$ be the corresponding set of points at infinity $r_{i}(\infty) \Gamma$ and let $C s p$ be the set of points at infinity $\bigcup_{i=1}^{k} C s p_{i}$. Then $C s p$ intersected with $\mathfrak{Q}_{\mathfrak{q}}$ seen as a subset of $\partial_{\infty} \mathcal{P}_{n+1}\left(L_{\mathfrak{q}}\right)$ is $\mathfrak{Q}_{\mathfrak{q}} \cap \mathbb{Q}^{n}$. In particular $\Omega \cap \mathbb{Q}^{n}=\Omega \cap C s p$, and it can be seen as a subset of $U_{+}(\varrho)$. For each $w \in C \operatorname{sp} \cap \Omega$ we use $\mathbf{u}_{w}$ to denote its corresponding unipotent element in $U_{+}(\varrho)$.

Note that to every point $w=r_{i}(\infty) \gamma$ in $C s p$ it is naturally associated a horoball $H b_{w}=H b\left(r_{i} \gamma\right)$ having it as a basepoint (see Section 2.1 for the definition of a horoball). Let $\varrho^{o p}$ be the geodesic ray opposite to $\varrho$. To every element $w \in C \operatorname{sp} \cap \Omega$ one can associate a weight $d_{w} \in \mathbb{R}_{+}$which is the distance from the horoball $H b\left(\varrho^{o p}\right)$ to the horoball $H b_{w}$. In $\Omega$ seen as a subset of $U_{+}(\varrho)$ one can then consider the set $\widetilde{\mathcal{S}}_{\Psi}^{0}(\Omega)$ of elements $\mathbf{u}$ such that

$$
\operatorname{dist}\left(\mathbf{u}, \mathbf{u}_{w}\right) \leq \Psi\left(d_{w}\right), \text { for infinitely many } w \in C s p,
$$

where dist is a left invariant metric on $U_{+}(\varrho)$ and $\Psi$ is an approximating function.

It turns out that, due to Lemma 4.1.1, the sets $\mathcal{S}_{\psi}(\Omega)$ and $\widetilde{\mathcal{S}}_{\Psi}^{0}(\Omega)$ are closely related, for an appropriate choice of the function $\Psi$. This relation is established using some explicit formulas obtained in Sections 3.3 and 3.4. See the double inclusion (29) and the whole discussion in Section 4.4 for details.

It suffices to study the set $\widetilde{\mathcal{S}}_{\Psi}^{0}(\Omega)$ from the point of view of the Hausdorff dimension. Moreover, it is not difficult to see that one can restrict the study to a subset $\widetilde{\mathcal{S}}_{\Psi}^{i}(\Omega)$ defined by replacing in (8) the set $C s p$ by the subset $C s p_{i}$.

In the particular cases when $\mathfrak{q}$ is positive definite or of signature $(1, n-1)$, $\mathcal{P}_{n+1}\left(L_{\mathfrak{q}}\right)$ is isometric to the hyperbolic space $\mathbb{H}^{n}$, and all the results in this paper follow from the results in $\left[\mathrm{BDV}_{2}, \S 8.3\right]$, generalizing previous results from $[\mathrm{HV}]$. We give an argument for the remaining cases. This argument actually works for the two previous cases too, with some slight modification. The inequality $\operatorname{dim}_{H} \widetilde{\mathcal{S}}_{\Psi}^{i}(\Omega) \leq \sigma(n-1)$ is not difficult to obtain. The main ingredient in its proof is the counting result Corollary 2.7.2, which gives an estimate of the number of balls $B\left(\mathbf{u}_{w}, \Psi\left(d_{w}\right)\right)$ in $U_{+}(\varrho)$ of a given size. This counting result itself follows from the equidistribution result Proposition 2.6.6.

For the converse inequality we use ubiquitous systems. We deduce from the equidistribution result Proposition 2.6.5 and the counting result Corollary 2.7.2 that the set of points $\Re=\left\{\mathbf{u}_{w} ; w \in C s p_{i} \cap \Omega\right\}$ together with the weight function $\varpi: \operatorname{Csp}_{i} \cap \Omega \rightarrow \mathbb{R}_{+}, \varpi(w)=d_{w}$, compose a local ubiquitous system with respect to an appropriate ubiquitous function and an appropriate increasing sequence of positive numbers, in the terminology of Section 4.3. We then use the properties of ubiquitous systems as developed in $\left[\mathrm{BDV}_{2}\right]$ to deduce the lower bound of the Hausdorff dimension of $\widetilde{\mathcal{S}}_{\Psi}^{i}(\Omega)$, as well as the other results. 
Some comments are necessary concerning the counting result Corollary 2.7.2. This statement corresponds in our case to the result in [Su, §6, Proposition 4], given for the rank one case. A generalization of Sullivan's result in the setting of geometrically finite Kleinian groups has been given in [HV]. A consequence of Corollary 2.7.2 is the following statement.

Corollary 1.2 (equidistribution of rational vectors on rational quadrics). Suppose that $\mathfrak{Q}_{\mathfrak{q}} \cap \mathbb{Q} \neq \emptyset$. Let $\Omega$ be a relatively compact open subset of $\mathfrak{Q}_{\mathfrak{q}}$ such that its closure does not intersect $T_{\bar{x}_{0}} \mathfrak{Q}_{\mathfrak{q}}$ for some $\bar{x}_{0} \in \mathfrak{Q}_{\mathfrak{q}}$. Let $a>1$. For every open subset $\mathcal{O}$ of $\Omega$ we denote by $N(k ; \mathcal{O})$ the cardinal of the set of rational vectors

$$
\left\{\frac{1}{q} \bar{p} \in \mathbb{Q}^{n} \cap \mathcal{O} ;|q| \in\left[a^{k}, a^{k+1}\right)\right\} .
$$

For any $a \geq a_{0}(\mathfrak{q}, \Omega)$ we have that

$\mathbf{K}_{1} a^{(k+1)(n-1)} v(\mathcal{O}) \leq N(k ; \mathcal{O}) \leq \mathbf{K}_{2} a^{(k+1)(n-1)} v(\mathcal{O})$, for every $k \geq k_{0}(\mathcal{O}, \Omega)$,

where $v$ is the canonical measure on $\mathfrak{Q}_{\mathfrak{q}}$ and $\mathbf{K}_{i}=\mathbf{K}_{i}(\mathfrak{q}, \Omega)$.

It is worth mentioning that our methods cannot be used to obtain either Khintchine type results or results on badly approximable vectors in $\mathfrak{Q}_{\mathfrak{q}}$ or any other type of results concerning vectors approximable nearly as well as the generic vectors in $\mathbb{R}^{n}$. This can be seen for instance by applying the logarithm law ([Su], $\left[\mathrm{KM}_{1}\right]$ ) in our setting. It implies that for every $\varepsilon>0$, for almost every $\bar{x} \in \mathfrak{Q}_{\mathfrak{q}}$, we have

$$
\left\|\bar{x}-\frac{1}{q} \bar{p}\right\| \geq \frac{c_{1}(\bar{x})}{q(\ln q)^{\frac{1}{n-1}+\varepsilon}}>\frac{1}{q^{1+\frac{1}{n}}}, \forall \frac{1}{q} \bar{p} \in \mathfrak{Q}_{\mathfrak{q}} .
$$

Consequently, for almost all $\bar{x} \in \mathfrak{Q}_{\mathfrak{q}}$ the rational approximants are outside $\mathfrak{Q}_{\mathfrak{q}}$. It seems that in order to study badly approximable and Khintchine type approximable vectors in $\mathfrak{Q}_{\mathfrak{q}}$, the study of the intrinsic geometry of $\mathcal{V}$ is not sufficient, and one has to consider also the "ambient" geometry of $\mathcal{T}_{n+1}=\mathcal{P}_{n+1} / S L(n+1, \mathbb{Z})$, where $\mathcal{P}_{n+1}$ is the symmetric space of positive definite quadratic forms on $\mathbb{R}^{n+1}$ of determinant 1 in the canonical basis. The locally symmetric space $\mathcal{T}_{n+1}$ contains a proper embedding of $\mathcal{V}[\mathrm{Bo}, \S 5]$.

\subsection{Rays moving away in the cusp}

We consider again the set $\widetilde{\mathcal{S}}_{\Psi}^{i}(\Omega)$ defined in Section 1.3. Without loss of generality we may assume that $\Omega=U_{+}(\varrho)$ and we may denote the corresponding set simply by $\widetilde{\mathcal{S}}_{\Psi}^{i}$. This set can be related to a set of geodesic rays moving away in the cusp for infinitely many times $t$ at depth at least $t-\phi(t)$, where the depth is measured with respect to the ray $\bar{r}_{i}$ and $\phi:[a,+\infty) \rightarrow[b,+\infty)$ is a function depending on the function $\Psi$. The results on the Hausdorff dimension of the set $\widetilde{\mathcal{S}}_{\Psi}^{i}$ can be thereby 
translated in terms of this set of rays. To simplify the exposition we present here a particular case of the results that can be obtained with such an argument, the general statements can be found in Section 5 .

Let $\beta \in(0,1)$ and define

$\mathcal{R}_{\beta}=\left\{\mathbf{u} \in U_{+}(\varrho) ; f_{\bar{r}_{i}}(\operatorname{proj}(\varrho(t) \mathbf{u})) \leq-\beta t\right.$ infinitely many times as $\left.t \rightarrow \infty\right\}$.

Above we have resumed the notation in Section 1.3, proj denotes the projection of $\mathcal{P}_{n+1}\left(L_{\mathfrak{q}}\right)$ onto $\mathcal{V}$ and $f_{\bar{r}_{i}}$ denotes the Busemann function of the ray $\bar{r}_{i}$ in $\mathcal{V}$, as defined in Section 2.1.

Note that $\mathcal{R}_{\beta}$ can also be seen as a set of geodesic rays, by identifying each $\mathbf{u}$ to the ray $\varrho \mathbf{u}$. The condition defining $\mathcal{R}_{\beta}$ means that for infinitely many times $t$ the projection onto $\mathcal{V}$ of the geodesic ray $\varrho \mathbf{u}$ goes into the cusp at depth at least $\beta t$, the depth into the cusp being measured with respect to the ray $\bar{r}_{i}$. We also consider a subset of $\mathcal{R}_{\beta}$, representing the rays which in some sense do not go deeper than $\beta t$ in the cusp as $t \rightarrow \infty$ :

$$
\mathcal{E} \mathcal{R}_{\beta}=\mathcal{R}_{\beta} \backslash \bigcup_{\beta^{\prime}>\beta} \mathcal{R}_{\beta^{\prime}}=\left\{\mathbf{u} \in \mathcal{R}_{\beta} ; \limsup _{t \rightarrow+\infty} \frac{-f_{\bar{r}_{i}}(\operatorname{proj}(\varrho(t) \mathbf{u}))}{t}=\beta\right\} .
$$

Theorem 1.3 (Corollary 5.1.5). For any $\beta \in(0,1)$,

$$
\begin{aligned}
\operatorname{dim}_{H} \mathcal{R}_{\beta} & =\operatorname{dim}_{H} \mathcal{E R}_{\beta}=(1-\beta) \operatorname{dim} U_{+}(\varrho) \\
& =d \text { and } \mathcal{H}^{d}\left(\mathcal{R}_{\beta}\right)=\mathcal{H}^{d}\left(\mathcal{E} \mathcal{R}_{\beta}\right)=\infty
\end{aligned}
$$

For a discussion of the cases $\beta=0,1$ see Section 5.1.

A natural question to ask is whether other results on the Hausdorff dimension and measure of sets of very well approximable vectors have an interpretation in terms of rays moving away in the cusp of some locally symmetric space. We establish such an interpretation for the formulas (1) and (4). Most likely this can be done in other cases as well. For the two formulas that we discuss the appropriate symmetric space is $\mathcal{P}_{n+1}$, and the appropriate locally symmetric space is $\mathcal{T}_{n+1}=\mathcal{P}_{n+1} / S L(n+1, \mathbb{Z})$. Let proj be the projection of $\mathcal{P}_{n+1}$ onto $\mathcal{T}_{n+1}$. Let $r_{1}$ and $r_{n}$ be the geodesic rays in $\mathcal{P}_{n+1}$ defined as in (19). The ray $r_{i}, i=1, n$, projects onto a geodesic ray $\bar{r}_{i}$ in $\mathcal{T}_{n+1}$. We define the set

$\mathcal{R}_{\beta}^{i}=\left\{\mathbf{u} \in U_{+}\left(r_{i}\right) ; f_{\bar{r}_{i}}\left(\operatorname{proj}\left(r_{i}(t) \mathbf{u}\right)\right) \leq-\beta t\right.$ infinitely many times as $\left.t \rightarrow \infty\right\}$,

where $i=1, n$, and $\beta \in(0,1)$. We also consider the subset

$$
\mathcal{E R}_{\beta}^{i}=\mathcal{R}_{\beta}^{i} \backslash \bigcup_{\beta^{\prime}>\beta} \mathcal{R}_{\beta^{\prime}}^{i}=\left\{\mathbf{u} \in \mathcal{R}_{\beta}^{i} ; \limsup _{t \rightarrow \infty} \frac{-f_{\bar{r}_{i}}\left(\operatorname{proj}\left(r_{i}(t) \mathbf{u}\right)\right)}{t}=\beta\right\} .
$$

Formula (1) implies the following. 
Theorem 1.4 (Corollary 5.2.3). For any $\beta \in(0,1)$,

$$
\begin{aligned}
\operatorname{dim}_{H} \mathcal{R}_{\beta}^{i} & =\operatorname{dim}_{H} \mathcal{E R}_{\beta}^{i}=(1-\beta) \operatorname{dim} U_{+}\left(r_{i}\right) \\
& =d \text { and } \mathcal{H}^{d}\left(\mathcal{R}_{\beta}^{i}\right)=\mathcal{H}^{d}\left(\mathcal{E R}_{\beta}^{i}\right)=\infty, i=1, n .
\end{aligned}
$$

Formula (4) also can be expressed in terms of sets of rays moving away in the cusp, but the situation slightly changes. In this case the ray in the cusp with respect to which the depth is measured and the rays whose behavior is studied are not in the same orbit of $S L(n+1, \mathbb{R})$, or in the terminology of Section 2.4, they do not have the same slope. This explains why in this case the parameter $\beta$ does not get near to 1 , but is bounded by a smaller constant depending on the two slopes. More precisely, we define for every $\beta \in\left(0, \frac{1}{n}\right)$

$\mathcal{R}_{\beta}^{1 n}=\left\{\mathbf{u} \in U_{+}\left(r_{1}\right) ; f_{\bar{r}_{n}}\left(\operatorname{proj}\left(r_{1}(t) \mathbf{u}\right)\right) \leq-\beta t\right.$ infinitely many times as $\left.t \rightarrow \infty\right\}$.

Let $\mathcal{E R}_{\beta}^{1 n}=\mathcal{R}_{\beta}^{1 n} \backslash \bigcup_{\beta^{\prime}>\beta} \mathcal{R}_{\beta^{\prime}}^{1 n}$. The sets $\mathcal{R}_{\beta}^{n 1}$ and $\mathcal{E R}_{\beta}^{n 1}$ can be defined similarly by intertwining 1 and $n$.

Theorem 1.5 (Corollary 5.3.4). For any $\beta \in\left(0, \frac{1}{n}\right)$,

$$
\begin{aligned}
\operatorname{dim}_{H} \mathcal{R}_{\beta}^{i j} & =\operatorname{dim}_{H} \mathcal{E R}_{\beta}^{i j}=(1-\beta) \operatorname{dim} U_{+}\left(r_{i}\right) \\
& =d \text { and } \mathcal{H}^{d}\left(\mathcal{R}_{\beta}^{i j}\right)=\mathcal{H}^{d}\left(\mathcal{E R}_{\beta}^{i j}\right)=\infty,\{i, j\}=\{1, n\} .
\end{aligned}
$$

For the cases $\beta=0, \frac{1}{n}$, see Section 5.3.

\subsection{Open question}

Theorems 1.3, 1.4 and 1.5 suggest that there might be a general formula for the Hausdorff dimension of the set of rays moving away into the cusp at linear depth. This justifies the following question.

Let $X$ be a symmetric space of non-compact type without Euclidean factors, let $G$ be the connected semisimple group of isometries of $X$, let $\Gamma$ be a non-uniform irreducible lattice of isometries of $X$, let $\mathcal{V}=X / \Gamma$ and let proj be the projection of $X$ onto $\mathcal{V}$. Consider $\varrho$ a geodesic ray in $X, \bar{r}$ a geodesic ray in $\mathcal{V}$ and $r$ a lift of $\bar{r}$ in $X$. The ray $r$ is contained in some Weyl chamber of vertex $r(0)$. In this same Weyl chamber there exists a unique ray $\varrho_{1}$ of vertex $r(0)$ and contained in the orbit $\varrho G$. The Busemann function $f_{r}$ restricted to $\varrho_{1}$ has the form $-\beta_{0} t$ for some $\beta_{0} \geq 0$. This implies that, as soon as $\beta_{0}>0, \operatorname{proj}\left(\varrho_{1}\right)$ moves away in the cusp of $\mathcal{V}$ and the depth at which it moves away at time $t$ measured with respect to the ray $\bar{r}$ is $\beta_{0} t$. Note that among all the geodesic rays in $\varrho G$ with origin on the horosphere $H(r)$, the ray $\varrho_{1}$ has the maximal depth at moment $t$ with respect to $\bar{r}$. 
Question 1.6. For every $\beta \in\left(0, \beta_{0}\right)$, consider the set $\mathcal{R}_{\beta}=\left\{\mathbf{u} \in U_{+}(\varrho) ;-f_{\bar{r}}(\varrho(t) \mathbf{u}) \geq \beta t\right.$ infinitely many times as $\left.t \rightarrow \infty\right\}$.

Is it true that $d=\operatorname{dim}_{H} \mathcal{R}_{\beta}=(1-\beta) \operatorname{dim} U_{+}(\varrho)$ and that $\mathcal{H}^{d}\left(\mathcal{R}_{\beta}\right)=\infty$ ?

\subsection{Organization of the paper}

Section 2 contains preliminaries on horoballs, symmetric spaces and semisimple groups. The equidistribution results Proposition 2.6.5 and Proposition 2.6.6 in Section 2.6 play an important part in our arguments. In particular the latter implies the counting results Proposition 2.7.1 and Corollary 2.7.2.

In Section 3 are given the formulas for the Busemann functions in the ambient symmetric space $\mathcal{P}_{n+1}$ as well as in the symmetric space associated to the quadric, $\mathcal{P}_{n+1}\left(L_{\mathfrak{q}}\right)$. In Sections 3.4 and 3.5 we study the geometry of horoballs of $\mathcal{P}_{n+1}\left(L_{\mathfrak{q}}\right)$. The obtained results together with the counting result Corollary 2.7.2 yield the equidistribution of rational vectors on rational quadrics as formulated in Corollary 1.2, and also a more general result, Proposition 3.4.4.

Section 4 contains the proof of Theorem 1.1. The notion of ubiquitous system is recalled in Section 4.3. In Section 4.4 we show the relation between the set $\mathcal{S}_{\psi}\left(\mathfrak{Q}_{\mathfrak{q}}\right)$ and the set of unipotents $\widetilde{\mathcal{S}}_{\Psi}^{0}$. We end our argument in Section 4.5 by exhibiting a local ubiquitous system and applying results from $\left[\mathrm{BDV}_{2}\right]$.

In Section 5 we prove results on the Hausdorff dimension and measure of sets of locally geodesic rays moving away in the cusp of a locally symmetric space. In Section 5.1 we study the case of rays of type $\wp$ in the locally symmetric space $\mathcal{P}_{n+1}\left(L_{\mathfrak{q}}\right) / \Gamma$, where $\Gamma$ is an arbitrary lattice in $S O_{I}\left(L_{\mathfrak{q}}\right)$. In the other two sections we deduce from (1) and (4) respectively results about rays in the locally symmetric space $\mathcal{T}_{n+1}$.

\section{Preliminaries on (locally) symmetric spaces}

The reader acquainted with semisimple groups and symmetric spaces may skip Sections 2.1 to 2.5 and refer to them only when needed.

\subsection{Notation and conventions}

We denote by $\mathcal{P} \mathbb{Z}^{n}$ the set of primitive integer vectors in $\mathbb{R}^{n}$,

$$
\left\{\left(p_{1}, p_{2}, \ldots, p_{n}\right) \in \mathbb{Z}^{n} \backslash\{(0, \ldots 0)\} ; \operatorname{gcd}\left(p_{1}, p_{2}, \ldots, p_{n}\right)=1\right\},
$$

and we denote by $\mathcal{P} \mathbb{Z}_{+}^{n}$ the subset

$$
\left\{\left(p_{1}, p_{2}, \ldots, p_{n}\right) \in \mathcal{P} \mathbb{Z}^{n} ; p_{n}>0 \text { or } p_{i}>0, p_{i+1}=\cdots=p_{n}=0\right\} .
$$


In a metric space $\left(X\right.$, dist), for any subset $A$ of $X$, we denote by $\mathcal{N}_{a}(A)$ the set

$$
\{x \in X ; \operatorname{dist}(x, A)<a\} .
$$

When $A=\left\{x_{0}\right\}$ then $\mathcal{N}_{a}(A)$ becomes an open ball and we use the notation $B\left(x_{0}, a\right)$.

We denote by $\operatorname{diag}\left(a_{1}, a_{2}, \ldots, a_{n}\right)$ the diagonal matrix having entries $a_{1}, a_{2}, \ldots, a_{n}$ on the diagonal. In the particular case when $a_{1}=\cdots=a_{k}=1$ and $a_{k+1}=\cdots=a_{k+\ell}=-1, k+\ell=n$, we denote by $I_{k, \ell}$ the diagonal matrix. We denote by $I d_{n}$ the identity matrix.

Throughout by line we mean a 1-dimensional linear (sub)space.

Let $A$ be a subset of $\mathbb{R}^{n}$. We denote by $\mathbb{R} A$ the union of all the lines intersecting $A$. We denote by $\mathbb{P} A$ the image of $\mathbb{R} A$ in $\mathbb{P}^{n-1} \mathbb{R}$. If $B \subset \mathbb{P}^{n-1} \mathbb{R}$ we denote by $\mathbb{R} B$ the subset in $\mathbb{R}^{n}$ which is union of all lines contained in $B$.

We denote by $\left\langle v_{1}, \ldots, v_{k}\right\rangle$ the linear subspace generated by the vectors $v_{1}, \ldots, v_{k}$.

Given two functions $f$ and $g$ with values in $\mathbb{R}$, we write $f \ll g$ if $f(x) \leq$ $C \cdot g(x)$, for every $x$, where $C>0$ is a universal constant. We write $f \asymp g$ if both $f \ll g$ and $f \gg g$ hold. We write $f \sim g$ if $\frac{f(x)}{g(x)} \rightarrow 1$ when $x \rightarrow \infty$. We denote by $\|f\|_{\infty}$ the supremum norm of the function $f$.

If $G$ is a group, we denote by $Z(G)$ its center $\{z \in G ; z g=g z, \forall g \in G\}$. If $H$ is a subgroup of $G$ we denote by $C_{G}(H)$ the center of $H$ in $G$, that is the group $\{z \in G ; z h=h z, \forall h \in H\}$.

If $G$ is a topological group, we denote by $G_{e}$ its connected component containing the identity.

Let $G$ be a Lie group. A lattice in $G$ is a discrete subgroup $\Gamma$ of $G$ such that $G / \Gamma$ has a finite $G$-invariant measure induced by the Haar measure on $G$. If $G / \Gamma$ is compact, the lattice is called uniform, otherwise it is called non-uniform.

If a group $G$ acts on a space $X$, for every point $x \in X$ we denote by $G_{x}$ the stabilizer of $x$ in $G$, that is the subgroup $\{g \in G ; g x=x\}$.

Let $X$ be a complete Riemannian manifold of non-positive curvature. Two geodesic rays in $X$ are called asymptotic if they are at finite Hausdorff distance one from the other. This defines an equivalence relation $\sim$ on the set $\mathcal{R}$ of all geodesic rays in $X$. The boundary at infinity of $X$ is the quotient $\mathcal{R} / \sim$. It is usually denoted by $\partial_{\infty} X$. Given $\xi \in \partial_{\infty} X$ and a geodesic ray $r$ in the equivalence class $\xi$, one writes $r(\infty)=\xi$.

Let $r$ be a geodesic ray in $X$. The Busemann function associated to $r$ is the function

$$
f_{r}: X \rightarrow \mathbb{R}, f_{r}(x)=\lim _{t \rightarrow \infty}[\operatorname{dist}(x, r(t))-t] .
$$

Since the function $t \rightarrow \operatorname{dist}(x, r(t))-t$ is non-increasing and bounded, the limit exists. The level hypersurfaces $H_{a}(r)=\left\{x \in X ; f_{r}(x)=a\right\}$ are called horospheres, the sublevel sets $H b_{a}(r)=\left\{x \in X ; f_{r}(x) \leq a\right\}$ are called closed 
horoballs and their interiors, $\mathrm{Hbo}_{a}(r)$, are called open horoballs. For $a=0$ we use the notation $H(r)$ for the horosphere, and $H b(r), H b o(r)$ for the closed and open horoball, respectively.

Suppose moreover that $X$ is simply connected.

Given an arbitrary point $x \in X$ and an arbitrary point at infinity $\xi \in \partial_{\infty} X$, there exists a unique geodesic ray $r$ with $r(0)=x$ and $r(\infty)=\xi$.

The Busemann functions of two asymptotic rays in $X$ differ by a constant $[\mathrm{BH}]$. Therefore we shall sometimes call them Busemann functions of basepoint $\xi$, where $\xi$ is the common point at infinity of the two rays. The families of horoballs and horospheres are the same for the two rays. We shall say that they are horoballs and horospheres of basepoint $\xi$.

Two points $\xi$ and $\zeta$ in $\partial_{\infty} X$ are said to be opposite if there exists a complete geodesic $\mathfrak{G}$ such that the point at infinity of $\left.\mathfrak{G}\right|_{[0,+\infty)}$ is $\xi$ and the point at infinity of $\left.\mathfrak{G}\right|_{(-\infty, 0]}$ is $\zeta$.

Definition 2.1.1. The oriented distance odist $\left(H b\left(r_{1}\right), H b\left(r_{2}\right)\right)$ between two horoballs $H b\left(r_{1}\right)$ and $H b\left(r_{2}\right)$ of opposite basepoints is $\inf _{x \in H b\left(r_{2}\right)} f_{r_{1}}(x)$.

\subsection{Semisimple groups and symmetric spaces}

Henceforth by $X$ we denote a symmetric space of non-compact type without Euclidean factors, and by $G$ the connected component of the identity in its group of isometries. Then $G$ is a semisimple Lie group. We identify the symmetric space $X$ with $K \backslash G$, where $K$ is a maximal compact subgroup of $G$. Hence we consider the action of $G$ on $X$ by isometries to the right, and correspondingly we consider the action of $G$ on itself by isometries to the right (with respect to the proper metric), and the action by conjugation also to the right, i.e. $a: G \rightarrow$ Aut $(G), a\left(g_{0}\right)(g)=g_{0}^{-1} g g_{0}$. For the theory of symmetric spaces and associated semisimple groups we refer to $[\mathrm{He}]$.

We recall that every connected semisimple real Lie group is isomorphic to the identity component of the real Lie group of real points of a semisimple algebraic group. Therefore, one can always talk about polynomial, rational and bi-rational maps on $G$. Moreover $G$ has a faithful embedding $f: G \rightarrow S L(n, \mathbb{R})$ such that $f(G)^{T}=f(G)$ and $f(K)=f(G) \cap O(n, \mathbb{R})$. Details can be found for instance in [OV], [Mo] or in [Ra].

Notation: We denote $\operatorname{dist}(e, g)$ by $|g|$, where dist is the right invariant metric on $G$.

An element $g_{0}$ in $S L(n, \mathbb{R})$ is hyperbolic if there exists $g \in G L(n, \mathbb{R})$ such that $g g_{0} g^{-1}$ is diagonalizable with all the eigenvalues real positive.

All the Lie groups considered in the sequel are real Lie groups, unless otherwise stated. 
Consider a field $\mathbb{K} \subset \mathbb{R}$. We say that a Lie group $G$ is defined over $\mathbb{K}$ if $G$ has finitely many connected components and if its connected component of the identity coincides with the connected component of the identity of a real algebraic group defined over $\mathbb{K}$ [Wi, Definition 6.2].

A torus is a closed connected Lie subgroup of $S L(n, \mathbb{R})$ which is diagonalizable over $\mathbb{C}$, i.e. such that there exists $g \in G L(n, \mathbb{C})$ with the property that $g T g^{-1}$ is diagonal. A torus is called $\mathbb{K}$-split if it is defined over $\mathbb{K}$ and diagonalizable over $\mathbb{K}$, that is if there exists $g \in G L(n, \mathbb{K})$ with the property that $g T g^{-1}$ is diagonal.

A torus (and more generally a reductive group) is called $\mathbb{K}$-anisotropic if it is defined over $\mathbb{K}$ and if it does not contain any non-trivial $\mathbb{K}$-split torus. Note that a $\mathbb{Q}$-anisotropic torus $T$ has the property that the set of its integer points $T_{\mathbb{Z}}$ is a lattice in it [Bo].

Conventions: Henceforth by torus we mean a non-trivial $\mathbb{R}$-split torus. The only exception is when we talk about $\mathbb{K}$-anisotropic torus, in which case the word keeps its general meaning. By wall/Weyl chamber we mean a closed wall/Weyl chamber. By its relative interior we mean the open wall/Weyl chamber.

We call singular torus in $G$ a torus $A_{0}$ which, in every maximal torus $A$ containing it, can be written as $\bigcap_{\lambda \in \Lambda} \operatorname{ker} \lambda$, where $\Lambda$ is a non-empty set of roots on $A$. Any such torus is a union of walls of Weyl chambers.

Let $\triangleleft A_{0}$ be a wall or a Weyl chamber in the torus $A_{0}$, and let $\triangleleft A_{0}^{o p}$ be the opposite wall. We consider the parabolic group corresponding to $\triangleleft A_{0}$,

$$
P\left(\triangleleft A_{0}\right)=\left\{g \in G ; \sup _{n \in \mathbb{N}}\left|\mathbf{a}^{n} g \mathbf{a}^{-n}\right|<+\infty, \forall \mathbf{a} \in \triangleleft A_{0}\right\},
$$

and the unipotent group corresponding to $\triangleleft A_{0}$,

$$
U\left(\triangleleft A_{0}\right)=\left\{g \in G ; \lim _{n \rightarrow \infty} \mathbf{a}^{n} g \mathbf{a}^{-n}=e, \forall \mathbf{a} \text { in the relative interior of } \triangleleft A_{0}\right\} .
$$

We denote $U\left(\triangleleft A_{0}^{o p}\right)$ by $U_{+}\left(\triangleleft A_{0}\right)$.

We have that $P\left(\triangleleft A_{0}\right)=C_{G}\left(A_{0}\right) U\left(\triangleleft A_{0}\right)=U\left(\triangleleft A_{0}\right) C_{G}\left(A_{0}\right), U\left(\triangleleft A_{0}\right)$ is the unipotent radical of $P\left(\triangleleft A_{0}\right)$, and $P\left(\triangleleft A_{0}\right)$ is the normalizer of $U\left(\triangleleft A_{0}\right)$ in $G$. The center decomposes as $C_{G}\left(A_{0}\right)=M A_{0}=A_{0} M$, where $Z(M)$ is compact and $M / Z(M)$ is semisimple. It follows that

$$
P\left(\triangleleft A_{0}\right)=M A_{0} U\left(\triangleleft A_{0}\right)=U\left(\triangleleft A_{0}\right) A_{0} M,
$$

which is called the Langlands decomposition of $P\left(\triangleleft A_{0}\right)$.

Remark 2.2.1. The action of $M$ on $U\left(\triangleleft A_{0}\right)$ by conjugation preserves the Haar measure. 
Proof. Any semisimple connected Lie group coincides with its commutator subgroup (see for instance [OV, §1.4.1 and §4.1.3]), hence any linear representation of a semisimple group preserves the volume. Consequently $\operatorname{Ad}(M)$ restricted to the Lie algebra $\mathfrak{u}$ of $U$ preserves the volume, which yields the conclusion.

The Lie algebras $\mathfrak{u}$ and $\mathfrak{u}_{+}$of $U\left(\triangleleft A_{0}\right)$ and $U_{+}\left(\triangleleft A_{0}\right)$ decompose into proper subspaces for $A d\left(A_{0}\right), \mathfrak{u}=\bigoplus_{\lambda\left(\triangleleft A_{0}\right)>1} \mathfrak{u}_{\lambda}$ and $\mathfrak{u}_{+}=\bigoplus_{\lambda\left(\triangleleft A_{0}\right)<1} \mathfrak{u}_{\lambda}$, respectively. Here $\lambda\left(\triangleleft A_{0}\right)>1$ signifies that $\lambda>1$ when restricted to the relative interior of $\triangleleft A_{0}$.

The sets $P\left(\triangleleft A_{0}\right) U_{+}\left(\triangleleft A_{0}\right)$ and $U_{+}\left(\triangleleft A_{0}\right) P\left(\triangleleft A_{0}\right)$ are open and Zariski dense in $G$. Therefore they both give coordinate systems in $G$ near $e$.

Suppose that the group $G$ is defined over $\mathbb{Q}$, that $A_{0}$ is a $\mathbb{Q}$-split torus and that $\triangleleft A_{0}$ is a $\mathbb{Q}$-wall or a $\mathbb{Q}$-Weyl chamber in it. Then $C_{G}\left(A_{0}\right)$ and $U\left(\triangleleft A_{0}\right)$ are also defined over $\mathbb{Q}$. Moreover $C_{G}\left(A_{0}\right)=M^{\prime} A_{0}=A_{0} M^{\prime}$, where $M^{\prime}$ is defined over $\mathbb{Q}, Z\left(M^{\prime}\right)_{e}$ is a $\mathbb{Q}$-anisotropic torus and $M^{\prime} / Z\left(M^{\prime}\right)_{e}$ is semisimple. Recall that in this case $\Gamma=G_{\mathbb{Z}}$ is a lattice in $G$, that $U\left(\triangleleft A_{0}\right) \cap \Gamma$ is a uniform lattice and that $M^{\prime} \cap \Gamma$ is a lattice in $M^{\prime}$.

For details on the previous results we refer to [Bo], [Ra] and [Wi].

We recall that a flat in $X$ is a totally geodesically embedded copy of an Euclidean space in $X$, and that a maximal flat is a flat which is maximal with respect to the inclusion. Every maximal flat $F$ is the orbit of a maximal torus $A$. Given a point $x \in F$, a Weyl chamber or $a$ wall with vertex $x$ is a set of type $x \triangleleft A_{0}$, where $\triangleleft A_{0}$ is a Weyl chamber or respectively a wall in the torus $A$. A singular flat through $x$ is an orbit $x A_{0}$, where $A_{0}$ is a singular torus in $A$. In the particular case when $G$ is defined over $\mathbb{Q}, A, A_{0}$ are $\mathbb{Q}$-split, $\triangleleft A_{0}$ is a $\mathbb{Q}$-Weyl chamber or wall, the corresponding maximal/singular flat, Weyl chamber or wall are called $\mathbb{Q}$-maximal/singular flat, $\mathbb{Q}$-Weyl chamber and $\mathbb{Q}$-wall, respectively.

The group $G$ acts transitively on the collection of maximal flats, as well as on the collection of Weyl chambers in $X$. This is equivalent to saying that it acts transitively by conjugation on the collection of maximal tori and on the collection of Weyl chambers in $G$. The stabilizer in $G$ of a Weyl chamber $W_{0}$ in $X$ is a compact subgroup $K_{0}$. Therefore $K_{0} \backslash G$ can be identified with the fiber bundle of the Weyl chambers in $X$.

\subsection{Geodesic rays, Busemann functions}

Let $\mathcal{A}=\left(\mathbf{a}_{t}\right)$ be a one-parameter subgroup of $G$ composed of hyperbolic elements and let $\mathcal{A}^{+}$be the positive sub-semigroup $\left(\mathbf{a}_{t}\right)_{t \geq 0}$. Let $r$ be a geodesic ray in $X$ such that $r(t)=r(0) \mathbf{a}_{t}$ for every $t \geq 0$. We consider $A_{0}$ either the minimal singular torus containing $\mathcal{A}$ or, if no such torus exists, the unique maximal torus containing $\mathcal{A}$. We have the equality $C_{G}(\mathcal{A})=C_{G}\left(A_{0}\right)$. If $A_{0}$ has dimension one we call the one-parameter group $\mathcal{A}$, the semigroup $\mathcal{A}^{+}$and the geodesic ray $r$ maximal singular. 
Let $\triangleleft A_{0}$ be the wall/Weyl chamber containing $\mathcal{A}^{+} \backslash\{e\}$ in its relative interior. We denote $P\left(\triangleleft A_{0}\right), U\left(\triangleleft A_{0}\right)$ and $U_{+}\left(\triangleleft A_{0}\right)$ also by $P(r), U(r)$ and $U_{+}(r)$, respectively, and we call them the parabolic, the unipotent and the opposite (expanding) unipotent group of the ray $r$.

The parabolic group $P(r)$ decomposes as $P(r)=\mathcal{A} P^{0}(r)$, where $P^{0}(r)$ is a codimension 1 subgroup acting transitively with compact stabilizer on every horosphere $H_{a}(r)$. We call $P^{0}(r)$ the horospherical group of $r$.

The following simple lemma will be useful in the future.

Lemma 2.3.1. Let $r$ be a geodesic ray in the symmetric space $X$ and let $\mathfrak{G}$ be the unique geodesic containing it, parameterized by arc length such that $r=\left.\mathfrak{G}\right|_{[0,+\infty)}$. Let $P^{0}$ be the horospherical group of $r$. A function $\Psi: X \rightarrow \mathbb{R}$ which is invariant with respect to $P^{0}$ and such that $\Psi(\mathfrak{G}(t))=-t, \forall t \in \mathbb{R}$, coincides with $f_{r}$.

Proof. For every $x \in X$ there exists a unique $t \in \mathbb{R}$ and $p \in P^{0}$ such that $x=\mathfrak{G}(t) p$. We have $\Psi(x)=\Psi(\mathfrak{G}(t) p)=\Psi(\mathfrak{G}(t))=-t=f_{r}(\mathfrak{G}(t))=f_{r}(x)$.

Consider the particular case when $G$ is defined over $\mathbb{Q}$ and when $\mathcal{A}^{+}=\triangleleft A_{0}$ is a $\mathbb{Q}$-wall. By the discussion in the end of Section 2.2, the horospherical group $P^{0}$ equals $M^{\prime} U\left(\triangleleft A_{0}\right)=U\left(\triangleleft A_{0}\right) M^{\prime}$, where $M^{\prime}$ and $U\left(\triangleleft A_{0}\right)$ are defined over $\mathbb{Q}$.

\subsection{Boundary at infinity}

If $W$ is a Weyl chamber or a wall in $X$ then its boundary at infinity $W(\infty)$ is a spherical simplex in $\partial_{\infty} X$, also called spherical chamber or respectively spherical wall. These simplices cover $\partial_{\infty} X$ and determine a structure of spherical building on it ([Mo, Chapters 15,16], [BGS, Appendix 5]).

Let $W_{0}$ be an arbitrary Weyl chamber in $X$. The group $G$ acts on $\partial_{\infty} X$ on the right with fundamental domain $W_{0}(\infty)$. Given a point $\xi$ in the relative interior of a spherical wall $W(\infty)$, where $W=x \triangleleft A_{0}$, the stabilizer of $\xi$ is the stabilizer of the whole wall $W(\infty)$, and it is the parabolic group $P\left(\triangleleft A_{0}\right)$. Since any parabolic group acts transitively on $X$, it follows that for every point $x \in X$ there exists a wall $W_{x}$ of vertex $x$ and such that $W_{x}(\infty)=W(\infty)$.

Given a fixed Weyl chamber $W_{0}, \partial_{\infty} X / G$ can be identified with $W_{0}(\infty)$, and one can define a projection sl $: \partial_{\infty} X \rightarrow W_{0}(\infty)$. The image sl $(\xi)$ of every point $\xi$ in $\partial_{\infty} X$ is called the slope of $\xi$. The slope of a geodesic ray $r$ is the slope of $r(\infty)$.

Let $x_{0}$ be an arbitrary point in $X$ and let $K$ be the maximal compact subgroup fixing $x_{0}$. Given a wall $W$ with vertex $x_{0}$, its stabilizer $K_{W}$ in $K$ is contained in the stabilizer $K_{F}$ of the minimal singular flat containing $W$, and it fixes both $W$ and $F$ pointwise. The group $K$ acts transitively on the set of Weyl chambers of vertex $x_{0}$. Hence, given the stabilizer $K_{W_{0}}$ of a Weyl chamber $W_{0}$ of vertex $x_{0}$, the quotient 
$K_{W_{0}} \backslash K$ can be identified with the set of Weyl chambers of vertex $x_{0}$. In particular, by the previous remarks, $K$ acts transitively on the set of spherical chambers of $\partial_{\infty} X$, and every spherical chamber $W_{0}(\infty)$ can be seen as the quotient $\partial_{\infty} X / K$.

\subsection{Locally symmetric spaces}

Let $\Gamma$ be a lattice in $G$. Here we shall be mainly interested in non-uniform irreducible lattices in semisimple groups of real rank at least 2. By Margulis Arithmeticity Theorem [Wi] such a lattice $\Gamma$ is an arithmetic lattice of $\mathbb{Q}$-rank $\mathbf{r} \geq 1$. The quotient space $\mathcal{V}=X / \Gamma$ is a locally symmetric space. It contains finitely many totally geodesic Euclidean sectors $W_{1}, \cdots, W_{m}$, of dimension $\mathbf{r}$, eventually glued to each other along faces, such that $\mathcal{V}$ is at finite Hausdorff distance of the union $W_{1} \cup \cdots \cup W_{m}$. Every sector $W_{1}, \ldots, W_{m}$ is the projection of a $\mathbb{Q}$-Weyl chamber. The quotient $\mathcal{V}$ can have several topological ends if and only if $\mathbf{r}=1$. For details see $[\mathrm{BoS}]$ and $[\mathrm{Le}]$.

Notation: We denote by proj the projection of $X$ onto $\mathcal{V}$ and by $\operatorname{proj}_{G}$ the projection of $G$ onto $G / \Gamma$.

Given a geodesic ray $\bar{r}$ entering one of the sectors $W_{i}, i \in\{1, \ldots, m\}$, the depth into the end containing $W_{i}$ can be measured by the Busemann function $f_{\bar{r}}$ of $\bar{r}$. If $\bar{r}$ is a face of dimension one of $W_{i}, i \in\{1, \ldots, m\}$, then we call it a maximal singular cusp ray. Let $r$ be a lift of $\bar{r}$ in $X$.

Remarks 2.5.1. (1) For $a<0$ with $|a|$ large enough, the projection $\operatorname{proj}\left(H b_{a}(r)\right)$ is $H b_{a}(\bar{r})$.

(2) There exists $\alpha=\alpha(\bar{r})>0$ such that

$$
\lim _{a \rightarrow-\infty} \frac{\ln v o l H b_{a}(\bar{r})}{a}=\alpha .
$$

Proof. (1) Since the projection proj is a contraction, $f_{\bar{r}}(\operatorname{proj}(x)) \leq f_{r}(x), \forall x \in$ $X$. This implies that $\operatorname{proj}\left(H b_{a}(r)\right) \subset H b_{a}(\bar{r})$.

One can identify $\mathcal{V}$ with a fundamental domain of $\Gamma$ in $X$, contained in a Siegel set as in [Bo, Theorem 15.5]. Suppose that $r$ is chosen so that under this identification $\bar{r}$ becomes $r$. Obviously for $a<0$ with $|a|$ large enough, $H b_{a}(\bar{r})$ coincides with the trace of $H b_{a}(r)$ on the fundamental domain. This implies that $H b_{a}(\bar{r}) \subset \operatorname{proj}\left(H b_{a}(r)\right)$.

(2) follows by looking at the form of the Siegel set as given in [Bo, Theorem 15.5].

Suppose that $\Gamma$ is arithmetic. Then without loss of generality we may suppose that $G$ admits a $\mathbb{Q}$-structure such that $\bar{r}$ is the projection of a $\mathbb{Q}$-wall $r$. The horospherical group $P^{0}(r)$ can be written as $M^{\prime} U\left(\triangleleft A_{0}\right)=U\left(\triangleleft A_{0}\right) M^{\prime}$, with both $M^{\prime}$ and $U\left(\triangleleft A_{0}\right)$ defined over $\mathbb{Q}$. In what follows, we denote $P^{0}(r)$ and $U\left(\triangleleft A_{0}\right)$ 
simply by $P^{0}$ and respectively $U$. According to [Bo, Corollary 7.13], $P^{0} \cap \Gamma$ is commensurable to the semidirect product $(U \cap \Gamma)\left(M^{\prime} \cap \Gamma\right)$. Therefore $P^{0} /\left(P^{0} \cap \Gamma\right)$ and $P^{0} /(U \cap \Gamma)\left(M^{\prime} \cap \Gamma\right)$ have a common finite covering. Now given $\mathcal{D}$ a fundamental domain of $U$ with respect to $U \cap \Gamma$ and $\mathcal{F}$ a fundamental domain of $M^{\prime}$ with respect to $M^{\prime} \cap \Gamma$, the set $\mathcal{F D}$ is a fundamental domain of $P^{0}$ with respect to $(U \cap \Gamma)\left(M^{\prime} \cap \Gamma\right)$. Indeed:

- $\mathcal{F} \mathcal{D}(U \cap \Gamma)\left(M^{\prime} \cap \Gamma\right)=\mathcal{F} U\left(M^{\prime} \cap \Gamma\right)=\mathcal{F}\left(M^{\prime} \cap \Gamma\right) U=M^{\prime} U$;

- if $u \in U \cap \Gamma$ and $m \in M^{\prime} \cap \Gamma$ are such that for some $f_{i} \in \mathcal{F}$ and $d_{i} \in \mathcal{D}$, $i=1,2, f_{1} d_{1} u m=f_{2} d_{2}$, then $f_{1} m^{-1}\left(d_{1} u\right) m=f_{2} d_{2}$, whence $f_{1} m=f_{2}$ and $m^{-1}\left(d_{1} u\right) m=d_{2}$. The former equality implies that $m=e$, the latter implies that $u=e$.

\subsection{Equidistribution results}

Let $G$ be a connected semisimple Lie group without compact factors and with trivial center. Let $\mathcal{A}=\left(\mathbf{a}_{t}\right)$ be a one-parameter subgroup of $G$ composed of hyperbolic elements, and $\mathcal{A}^{+}=\left(\mathbf{a}_{t}\right)_{t \geq 0}$. Let $A_{0}$ be either the minimal singular torus containing $\mathcal{A}$ or the unique maximal torus containing $\mathcal{A}$, and $\triangleleft A_{0}$ its unique wall/Weyl chamber containing $\mathcal{A}^{+} \backslash\{e\}$ in its relative interior. Let $\mathfrak{C}=C_{G}\left(A_{0}\right)=C_{G}(\mathcal{A})$, $P=P\left(\triangleleft A_{0}\right), U=U\left(\triangleleft A_{0}\right)$ and $U_{+}=U_{+}\left(\triangleleft A_{0}\right)$, endowed with their Haar measures.

Notation: For every subset $S$ of $G$, we denote by $S_{t}$ the subset $a\left(\mathbf{a}_{-t}\right) S$. We denote by $S^{-1}$ the image of $S$ under the inversion in $G$.

For $p \in P$ fixed, we consider the (partially defined) map $\Psi_{p}$ from $U_{+}$to $U_{+}$, defined by

$$
\Psi_{p}\left(\mathbf{u}_{+}\right)=\mathbf{u}_{+}^{\prime} \text { such that } P \mathbf{u}_{+}=P \mathbf{u}_{+}^{\prime} p .
$$

Let $D_{p}$ be its maximal domain of definition. Associated to this map, we have the maps $\pi_{p}: D_{p} \rightarrow P, v_{p}: D_{p} \rightarrow U$ and $\kappa_{p}: D_{p} \rightarrow \mathfrak{C}$ defined by the relations

$$
\Psi_{p}\left(\mathbf{u}_{+}\right) p=\pi_{p}\left(\mathbf{u}_{+}\right) \mathbf{u}_{+} \text {and } \pi_{p}\left(\mathbf{u}_{+}\right)=v_{p}\left(\mathbf{u}_{+}\right) \kappa_{p}\left(\mathbf{u}_{+}\right) .
$$

Notation: For $\alpha>0$ we define $\mathcal{Q}_{\alpha}=\{p \in P ; p=u c, u \in U,|u| \leq \alpha, c \in$ $\mathfrak{C},|c| \leq \alpha\}$.

Lemma 2.6.1. (i) For any $p \in P$, the domain $D_{p}$ is an open Zariski dense subset of $U_{+}$and the map $\Psi_{p}$ is bi-rational. It satisfies the relation $a\left(\mathbf{a}_{t}\right) \circ \Psi_{p}=$ $\Psi_{a\left(\mathbf{a}_{t}\right)(p)} \circ a\left(\mathbf{a}_{t}\right)$, where $a: G \rightarrow A u t(G)$ is the action to the right of $G$ on itself by conjugation.

(ii) Let $K$ be a compact subset in $P$. The set $\bigcap_{p \in K} D_{p}$ contains a neighborhood of $e$. 


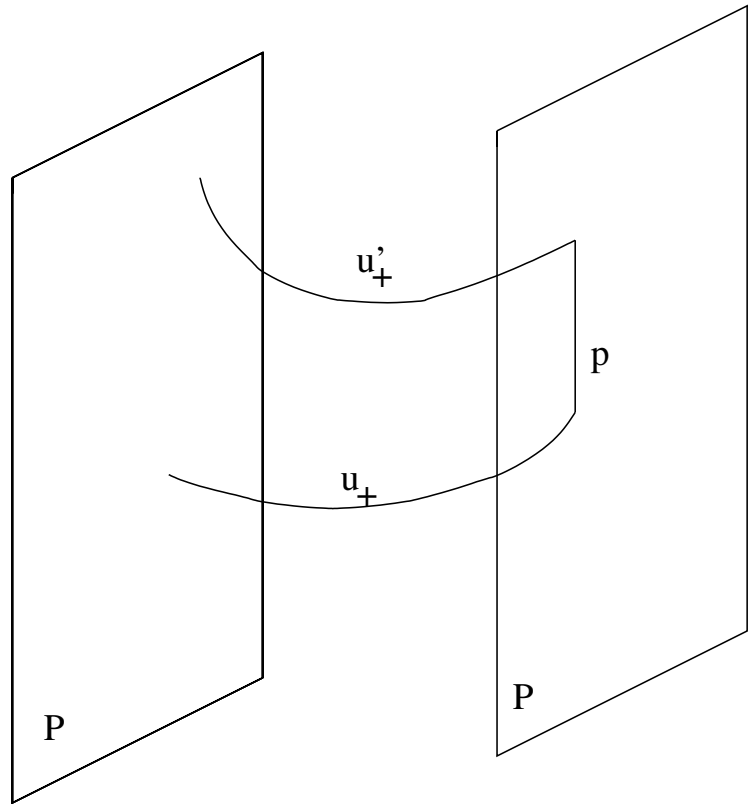

Fig. 1. The map $\Psi_{p}$

(iii) Let $K$ be a compact subset in $P$ and $\Omega$ an open subset in $U_{+}$. The intersection $\bigcap_{p \in K} \Psi_{p}\left(\Omega \cap D_{p}\right)$ is open.

(iv) The map $\Lambda_{p}: D_{p} \rightarrow U_{+}, \Lambda_{p}\left(\mathbf{u}_{+}\right)=\mathbf{u}_{+}^{-1} \Psi_{p}\left(\mathbf{u}_{+}\right)$tends to the constant map equal to the identity element e uniformly on compact subsets as $p \in \mathcal{Q}_{\alpha}$ and $\alpha \rightarrow 0$.

(v) The Jacobian of the map $\Psi_{p}$, which we denote by $\left|\frac{d \Psi_{p}}{d \mathbf{u}_{+}}\right|$, tends to the constant map equal to 1 uniformly on compact subsets as $p \in \mathcal{Q}_{\alpha}$ and $\alpha \rightarrow 0$.

(vi) The map $S_{\alpha}\left(\mathbf{u}_{+}\right)=\sup _{p \in \mathcal{Q}_{\alpha}}\left(\left|v_{p}\left(\mathbf{u}_{+}\right)\right|+\left|\kappa_{p}\left(\mathbf{u}_{+}\right)\right|\right)$tends to zero uniformly on compact subsets as $p \in \mathcal{Q}_{\alpha}$ and $\alpha \rightarrow 0$.

Proof. (i) Let $D_{p}=U_{+} \cap P U_{+} p$, which is an open Zariski dense subset of $U_{+}$. For every $\mathbf{u}_{+} \in D_{p}, \mathbf{u}_{+} p^{-1}=\bar{p} \overline{\mathbf{u}}_{+} \in P U_{+}$. It follows that $\Psi_{p}\left(\mathbf{u}_{+}\right)=\overline{\mathbf{u}}_{+}$. The map from $P U_{+}$to $U_{+}$defined by $\bar{p} \overline{\mathbf{u}}_{+} \rightarrow \overline{\mathbf{u}}_{+}$is a rational map. Hence the map $\Psi_{p}$ is rational. Moreover, since the converse map is $\Psi_{p^{-1}}$, the map $\Psi_{p}$ is bi-rational. The behavior of $\Psi_{p}$ with respect to the action of the group $\left(\mathbf{a}_{t}\right)$ can be deduced by applying $a\left(\mathbf{a}_{t}\right)$ in the first equality in (9).

(ii) Suppose that $\bigcap_{p \in K} D_{p}$ does not contain a neighborhood of $e$. Then there exists a sequence $\mathbf{u}_{n}^{+} \rightarrow e$ and a sequence $p_{n} \in K$ such that $\mathbf{u}_{n}^{+} p_{n}^{-1} \notin P U_{+}$for any $n \in \mathbb{N}$. A subsequence of $\mathbf{u}_{n}^{+} p_{n}^{-1}$ converges to some $p_{0} \in P \subset P U_{+}$. This contradicts the fact that $P U_{+}$is open. 
(iii) We prove that $\bigcup_{p \in K} \complement \Psi_{p}\left(\Omega \cap D_{p}\right)$ is closed. Let $\mathbf{u}_{n}^{+}$be a sequence in this set, converging to $\mathbf{u}_{+}$. For every $n \in \mathbb{N}$ there exists $p_{n} \in K$ such that $\mathbf{u}_{n}^{+} \notin \Psi_{p_{n}}\left(\Omega \cap D_{p_{n}}\right)$. Up to taking a subsequence, $p_{n}$ converges to $p \in K$. Suppose that $\mathbf{u}_{+} \in \Psi_{p}\left(\Omega \cap D_{p}\right)$, which is equivalent to the fact that $\mathbf{u}_{+} p \in P \Omega$. Since $P \Omega$ is an open set in $G$ and $\mathbf{u}_{n}^{+} p_{n} \rightarrow \mathbf{u}_{+} p$, for some $n$ large enough we have $\mathbf{u}_{n}^{+} p_{n} \in P \Omega$, that is $\mathbf{u}_{n}^{+} \in \Psi_{p_{n}}\left(\Omega \cap D_{p_{n}}\right)$. This contradicts the hypothesis.

From its definition it is straightforward that when $p \in \mathcal{Q}_{\alpha}$ and $\alpha \rightarrow 0$ the map $\Psi_{p} \rightarrow I d$ uniformly in the $C^{1}$ topology on compact subsets. This implies (iv), (v) and (vi).

Let $\Omega$ be a relatively compact open neighborhood of $e$ in $U_{+}$and let $\alpha$ be a small positive number. We suppose that $\alpha \leq 1$ and that $\Omega$ is small enough to be contained in $\bigcap_{p \in \mathcal{Q}_{1} \cup \mathcal{Q}_{1}^{-1}} D_{p}$.

Definitions 2.6.2. The $(\alpha, \Omega)$-box of basepoint $g_{0}$ is the set

$$
\operatorname{Box}_{\alpha, \Omega}\left(g_{0}\right)=\bigcup_{p \in \mathcal{Q}_{\alpha}} \bigcup_{\mathbf{u}_{+} \in \Psi_{p}(\Omega)} \mathbf{u}_{+} p g_{0}
$$

We call

$$
\mathbf{a}_{t} \operatorname{Box}_{\alpha, \Omega}\left(g_{0}\right)=\bigcup_{p \in\left(\mathcal{Q}_{\alpha}\right)_{t}} \bigcup_{\mathbf{u}_{+} \in \Psi_{p}\left(\Omega_{t}\right)} \mathbf{u}_{+} p g_{t},
$$

where $g_{t}=\mathbf{a}_{t} g_{0}$, the t-pushed $(\alpha, \Omega)$-box.

We denote by $\delta(\alpha, \Omega)$ and $S(\alpha, \Omega)$ the maximal values, for $p \in \mathcal{Q}_{\alpha}$, of $\sup _{\mathbf{u}_{+} \in \Omega}|| \frac{d \Psi_{p}}{d \mathbf{u}_{+}}|-1|$ and of $\sup _{\mathbf{u}_{+} \in \Omega} S_{\alpha}\left(\mathbf{u}_{+}\right)$respectively.

Definitions 2.6.3. We call $\varepsilon$-base of $\Omega$ an open relatively compact neighborhood $\Omega_{\varepsilon}$ of $e$ in $U_{+}$such that $v(\Omega \triangle \Omega \mathcal{K}) \leq \varepsilon v(\Omega)$, for every nonempty $\mathcal{K} \subset \Omega_{\varepsilon}$, where $v$ is the Haar measure on $U_{+}$and $A \triangle B=(A \backslash B) \cup(B \backslash A)$.

We call $(\varepsilon, \alpha)$-base of $\Omega$ any subset of $U_{+}$of the form

$$
\Omega_{\varepsilon, \alpha}=\bigcap_{p \in \mathcal{Q}_{\alpha}} \Psi_{p^{-1}}\left(\Omega_{\varepsilon} \cap D_{p^{-1}}\right),
$$

where $\Omega_{\varepsilon}$ is an $\varepsilon$-base of $\Omega$. According to Lemma 2.6.1, (iii), $\Omega_{\varepsilon, \alpha}$ is an open relatively compact neighborhood of $e$.

We call Box $x_{\alpha, \Omega_{\varepsilon, \alpha}}\left(g_{0}\right)$ an $\varepsilon$-base of the box Box $\operatorname{Bos}_{\alpha, \Omega}\left(g_{0}\right)$. 


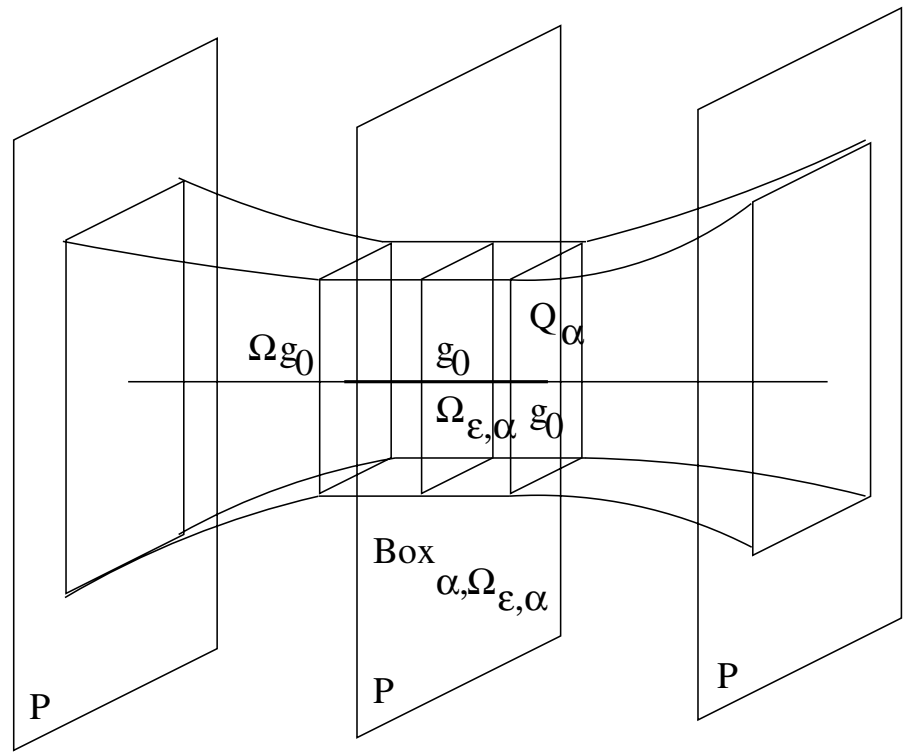

Fig. 2. A box and an $\varepsilon$-base of it

Notation: We denote $\oint_{D} f d \mu=\frac{1}{\mu(D)} \int_{D} f d \mu$.

Lemma 2.6.4. Let $f$ be a bounded uniformly continuous function on $G$, of modulus of continuity $\omega$. Let $\Omega$ be a sufficiently small relatively compact open neighborhood of e in $U_{+}$, let $\varepsilon$ be a small positive number and let $\Omega_{\varepsilon}$ be an $\varepsilon$-base of $\Omega$. For $\alpha>0$ sufficiently small we have that

$$
\begin{aligned}
& \left|\oint_{\Omega_{t}} f\left(\mathbf{u}_{+} y\right) d v\left(\mathbf{u}_{+}\right)-\oint_{\Omega_{t}} f\left(\mathbf{u}_{+} y^{\prime}\right) d v\left(\mathbf{u}_{+}\right)\right| \\
& \quad=O\left(\omega(S(\alpha, \Omega))+\|f\|_{\infty}\left[\varepsilon+\delta\left(\alpha, \Omega \Omega_{\varepsilon}\right)\right]\right),
\end{aligned}
$$

for every $g_{0} \in G$, every $t \in[0,+\infty)$ and every $y, y^{\prime}$ in the t-pushed $\varepsilon$-base $\mathbf{a}_{t} \operatorname{Box}_{\alpha, \Omega_{\varepsilon, \alpha}}\left(g_{0}\right)$.

Proof. We suppose that $\alpha<1$ and that $\Omega \subset \bigcap_{p \in \mathcal{Q}_{1} \cup \mathcal{Q}_{1}^{-1}} D_{p}$. It suffices to prove (10) for $y^{\prime}=g_{t}$. Since $y \in \mathbf{a}_{t} B o x_{\alpha, \Omega_{\varepsilon, \alpha}}\left(g_{0}\right)$, we may write $y=\mathbf{a}_{t} \widetilde{\mathbf{u}}_{+} p g_{0}$, where $p \in \mathcal{Q}_{\alpha}$ and $\widetilde{\mathbf{u}}_{+} \in \Psi_{p}\left(\Omega_{\varepsilon, \alpha}\right) \subset \Omega_{\varepsilon}$. Then

$$
\begin{aligned}
& \oint_{\Omega_{t}} f\left(\mathbf{u}_{+} y\right) d v\left(\mathbf{u}_{+}\right)=\oint_{\Omega_{t}} f\left(\mathbf{u}_{+} \mathbf{a}_{t} \widetilde{\mathbf{u}}_{+} p g_{0}\right) d v\left(\mathbf{u}_{+}\right) \\
& \quad=\oint_{\Omega} f\left(\mathbf{a}_{t} \eta_{+} \widetilde{\mathbf{u}}_{+} p g_{0}\right) d v\left(\eta_{+}\right)=\oint_{\Omega \widetilde{\mathbf{u}}_{+}} f\left(\mathbf{a}_{t} \eta_{+} p g_{0}\right) d v\left(\eta_{+}\right) .
\end{aligned}
$$

By the definition of $\Omega_{\varepsilon}$, we have that $v\left(\Omega \triangle \Omega \widetilde{\mathbf{u}}_{+}\right) \leq \varepsilon v(\Omega)$. It follows that

$$
\oint_{\Omega \widetilde{\mathbf{u}}_{+}} f\left(\mathbf{a}_{t} \eta_{+} p g_{0}\right) d v\left(\eta_{+}\right)=\oint_{\Omega} f\left(\mathbf{a}_{t} \eta_{+} p g_{0}\right) d v\left(\eta_{+}\right)+O\left(\varepsilon\|f\|_{\infty}\right) .
$$


We want to change the integration domain from $\Omega$ to $\Psi_{p}(\Omega)$. We consider $\alpha$ small enough so that $\Lambda_{p}(\Omega) \subset \Omega_{\varepsilon}$, for any $p \in \mathcal{Q}_{\alpha} \cup \mathcal{Q}_{\alpha}^{-1}$.

We have

$$
\Psi_{p}(\Omega)=\left\{\Psi_{p}\left(\mathbf{u}_{+}\right) ; \mathbf{u}_{+} \in \Omega\right\}=\left\{\mathbf{u}_{+} \Lambda_{p}\left(\mathbf{u}_{+}\right) ; \mathbf{u}_{+} \in \Omega\right\} \subset \Omega \Omega_{\varepsilon} .
$$

Therefore $v\left(\Psi_{p}(\Omega) \backslash \Omega\right) \leq \varepsilon v(\Omega)$.

We write $\Omega \backslash \Psi_{p}(\Omega)$ as the image under $\Psi_{p}$ of $\Psi_{p^{-1}}(\Omega) \backslash \Omega$. An argument similar to the previous implies that $\Psi_{p^{-1}}(\Omega) \subset \Omega \Omega_{\varepsilon}$, hence $v\left(\Psi_{p^{-1}}(\Omega) \backslash \Omega\right) \leq \varepsilon v(\Omega)$. Since the Jacobian of $\Psi_{p}$ differs from 1 by $O\left(\delta\left(\alpha, \Omega \Omega_{\varepsilon}\right)\right)$ on $\Psi_{p^{-1}}(\Omega)$, we may conclude that $v\left(\Omega \backslash \Psi_{p}(\Omega)\right) \leq \varepsilon v(\Omega)\left[1+\delta\left(\alpha, \Omega \Omega_{\varepsilon}\right)\right]$. Consequently

$$
\begin{aligned}
\oint_{\Omega} f\left(\mathbf{a}_{t} \eta_{+} p g_{0}\right) d v\left(\eta_{+}\right)= & \frac{1}{v(\Omega)} \int_{\Psi_{p}(\Omega)} f\left(\mathbf{a}_{t} \eta_{+} p g_{0}\right) d v\left(\eta_{+}\right) \\
& +O\left(\varepsilon\|f\|_{\infty}\left[1+\delta\left(\alpha, \Omega \Omega_{\varepsilon}\right)\right]\right) .
\end{aligned}
$$

With the change $\eta_{+}=\Psi_{p}\left(\eta_{+}^{\prime}\right)$ we may write

$$
\begin{aligned}
\frac{1}{v(\Omega)} \int_{\Psi_{p}(\Omega)} f\left(\mathbf{a}_{t} \eta_{+} p g_{0}\right) d v\left(\eta_{+}\right)= & \oint_{\Omega} f\left(\mathbf{a}_{t} \Psi_{p}\left(\eta_{+}^{\prime}\right) p g_{0}\right) d v\left(\eta_{+}^{\prime}\right) \\
& +O\left(\delta(\alpha, \Omega)\|f\|_{\infty}\right) .
\end{aligned}
$$

Using the notation in (9) we may write

$$
\oint_{\Omega} f\left(\mathbf{a}_{t} \Psi_{p}\left(\eta_{+}^{\prime}\right) p g_{0}\right) d v\left(\eta_{+}^{\prime}\right)=\oint_{\Omega} f\left(\mathbf{a}_{t} v_{p}\left(\eta_{+}^{\prime}\right) \kappa_{p}\left(\eta_{+}^{\prime}\right) \eta_{+}^{\prime} g_{0}\right) d v\left(\eta_{+}^{\prime}\right) .
$$

By the right invariance of the metric on $G$ we have that

$$
\begin{aligned}
& \operatorname{dist}\left(\mathbf{a}_{t} v_{p}\left(\eta_{+}^{\prime}\right) \kappa_{p}\left(\eta_{+}^{\prime}\right) \eta_{+}^{\prime} g_{0}, \mathbf{a}_{t} \eta_{+}^{\prime} g_{0}\right) \\
& =\operatorname{dist}\left(a\left(\mathbf{a}_{-t}\right)\left(v_{p}\left(\eta_{+}^{\prime}\right)\right) \kappa_{p}\left(\eta_{+}^{\prime}\right), e\right) \leq S(\alpha, \Omega) .
\end{aligned}
$$

Therefore the last integral is equal to

$$
\begin{aligned}
& \oint_{\Omega} f\left(\mathbf{a}_{t} \eta_{+}^{\prime} g_{0}\right) d v\left(\eta_{+}^{\prime}\right)+O(\omega(S(\alpha, \Omega))) \\
& =\oint_{\Omega_{t}} f\left(\mathbf{u}_{+} g_{t}\right) d v\left(\mathbf{u}_{+}\right)+O(\omega(S(\alpha, \Omega))) .
\end{aligned}
$$

Proposition 2.6.5. Let $\Gamma$ be an irreducible lattice in $G$ and let $f: G / \Gamma \rightarrow \mathbb{R}$ be a function which is uniformly continuous and bounded. Let $\left(\mathbf{a}_{t}\right)_{t \in \mathbb{R}}$ be a oneparameter group composed of hyperbolic elements and let $U_{+}$be the expanding unipotent subgroup corresponding to $\left(\mathbf{a}_{t}\right)_{t \geq 0}$. Let $\Omega$ be an open relatively compact set in $U_{+}$, with the property that there exists $\mathbf{u}_{0} \in \Omega^{-1}$ such that for any $t_{0} \in \mathbb{R}$, 
the family of sets $a\left(\mathbf{a}_{-t}\right)\left(\Omega \mathbf{u}_{0}\right), t \in\left[t_{0},+\infty\right)$, is a summing net for $U_{+}$, in the sense of $[P a, \S 4.15]$. For any $\bar{g}_{0} \in G / \Gamma$,

$$
\oint_{\Omega} f\left(\mathbf{a}_{t} \mathbf{u}_{+} \bar{g}_{0}\right) d \nu\left(\mathbf{u}_{+}\right) \rightarrow \oint_{G / \Gamma} f d \mu \text { as } t \rightarrow+\infty,
$$

where $\mu$ is the measure on $G / \Gamma$ induced by the Haar measure on $G$.

Proof. Step 1. We suppose that $\Omega$ is a neighborhood of $e$ contained in $\bigcap_{p \in \mathcal{Q}_{1} \cup \mathcal{Q}_{1}^{-1}} D_{p}$ and that $\mathbf{u}_{0}=e$. We denote by $\omega$ the modulus of continuity of $f$. We fix arbitrary $\bar{g}_{0} \in G / \Gamma$ and $\varepsilon$ small positive number. We consider $\Omega_{\varepsilon}$ an $\varepsilon$-base of $\Omega$ and $\alpha>0$ sufficiently small so that the conclusion of Lemma 2.6.4 holds, and also so that $\omega(S(\alpha, \Omega)) \leq \varepsilon$ and $\delta\left(\alpha, \Omega \Omega_{\varepsilon}\right) \leq \frac{\varepsilon}{\|f\|_{\infty}}$.

The group $U_{+}$acts ergodically on $G / \Gamma[\mathrm{Zi}, \S 2.2]$. This and the fact that the family of sets $\Omega_{t}=a\left(\mathbf{a}_{-t}\right)(\Omega)$ is a summing net implies that $\oint_{\Omega_{t}} f\left(\mathbf{u}_{+} \bar{g}\right) d v\left(\mathbf{u}_{+}\right)$, seen as a function of $\bar{g} \in G / \Gamma$, converges to $\oint_{G / \Gamma} f d \mu$ in $L^{2}(G / \Gamma)$ as $t \rightarrow \infty$ $[\mathrm{Pa}, \S 5]$. This implies that for the given $\varepsilon$ and for any small $\lambda>0$ there exists $T=T(\varepsilon, \lambda, \Omega)$ such that for every $t \geq T$ the set of points $\bar{g} \in G / \Gamma$ satisfying

$$
\left|\oint_{\Omega_{t}} f\left(\mathbf{u}_{+} \bar{g}\right) d v\left(\mathbf{u}_{+}\right)-\oint_{G / \Gamma} f d \mu\right| \geq \varepsilon
$$

has measure strictly smaller than $\lambda$. We take $\lambda$ to be the measure of the projection in $G / \Gamma$ of the $\varepsilon$-base $B o x_{\alpha, \Omega_{\varepsilon, \alpha}}\left(g_{0}\right)$. Hence, for every $t \geq T$, at least one point $\bar{y}$ in the projection of the $t$-pushed $\varepsilon$-base satisfies the inequality opposite to (11). This and Lemma 2.6.4 imply that for every $t \geq T$,

$$
\left|\oint_{\Omega_{t}} f\left(\mathbf{u}_{+} \bar{g}_{t}\right) d v\left(\mathbf{u}_{+}\right)-\oint_{G / \Gamma} f d \mu\right|<C \varepsilon,
$$

where $C=C\left(\|f\|_{\infty}\right)$ and $\bar{g}_{t}=\mathbf{a}_{t} \bar{g}_{0}$.

Step 2. We suppose that $\mathbf{u}_{0}=e$ and that $\Omega$ is an arbitrary open relatively compact neighborhood of $e$ in $U_{+}$satisfying the hypothesis and not necessarily contained in $\bigcap_{p \in \mathcal{Q}_{1} \cup \mathcal{Q}_{1}^{-1}} D_{p}$. There exists $\tau \in(0,+\infty)$ so that $\Omega_{-\tau}=a\left(\mathbf{a}_{\tau}\right)(\Omega) \subset$ $\bigcap_{p \in \mathcal{Q}_{1} \cup \mathcal{Q}_{1}^{-1}} D_{p}$. We apply the result obtained in Step 1 to $\Omega_{-\tau}$ and with a change of variables we obtain the same result for $\Omega$.

Step 3. We consider the general case. By Step 2 we have the conclusion of the Proposition for $\Omega \mathbf{u}_{0}$. This implies the conclusion for $\Omega$.

Proposition 2.6.6. Let $\Gamma$ be a lattice in $G$ and $f: G / \Gamma \rightarrow \mathbb{R}$ a bounded uniformly continuous function. Let $\left(\mathbf{a}_{t}\right)_{t \in \mathbb{R}}$ be a one-parameter group composed of hyperbolic elements and let $U_{+}$be the expanding unipotent subgroup corresponding to $\left(\mathbf{a}_{t}\right)_{t \geq 0}$ and $\mathfrak{C}$ be the center of the group in $G$. Let $\Omega$ be an open relatively compact set in $U_{+}$, with the same property as in Proposition 2.6 .5 and let $\Phi$ 
be a finite volume submanifold in $\mathfrak{C}$. We denote by $\vartheta$ the volume on $\Phi$. For any $\bar{g}_{0} \in G / \Gamma$,

$$
\oint_{\Phi \Omega} f\left(\mathbf{a}_{t} \mathbf{c} \mathbf{u}_{+} \bar{g}_{0}\right) d v\left(\mathbf{u}_{+}\right) d \vartheta(\mathbf{c}) \rightarrow \oint_{G / \Gamma} f d \mu \text { as } t \rightarrow+\infty,
$$

where $\mu$ is the measure on $G / \Gamma$ induced by the Haar measure on $G$.

Proof. We fix an arbitrary small positive number $\varepsilon$. There exists a compact subset $K=K(\varepsilon)$ in $\Phi$ such that $\vartheta(\Phi \backslash K)<\frac{\varepsilon}{2\|f\|_{\infty}} \vartheta(\Phi)$. It follows that

$$
\left|\oint_{\Phi \Omega} f\left(\mathbf{a}_{t} \mathbf{c} \mathbf{u}_{+} \bar{g}_{0}\right) d v\left(\mathbf{u}_{+}\right) d \vartheta(\mathbf{c})-\oint_{K \Omega} f\left(\mathbf{a}_{t} \mathbf{c} \mathbf{u}_{+} \bar{g}_{0}\right) d v\left(\mathbf{u}_{+}\right) d \vartheta(\mathbf{c})\right|<\varepsilon .
$$

Let $\omega$ be the modulus of continuity of $f$. Let $\delta>0$ be such that $\omega(\delta)<\varepsilon$. By compactness of $K$, there exist $\mathbf{k}_{1}, \ldots, \mathbf{k}_{m}$ in $K$ so that the set of balls $B\left(\mathbf{k}_{i}, \delta\right), i \in$ $\{1, \ldots, m\}$, is a cover of $K$. Thus, for every $\mathbf{c} \in K$ there exists $i \in\{1, \ldots, m\}$ such that $\operatorname{dist}\left(\mathbf{c}, \mathbf{k}_{i}\right)<\delta$, which by the right invariance of the distance and the fact that the projection is a contraction implies that $\operatorname{dist}\left(\mathbf{c a}_{t} \mathbf{u}_{+} \bar{g}_{0}, \mathbf{k}_{i} \mathbf{a}_{t} \mathbf{u}_{+} \bar{g}_{0}\right)<\delta$, for any $t \in \mathbb{R}, \mathbf{u}_{+} \in U_{+}$and $g_{0} \in G$. It follows that

$$
\left|f\left(\mathbf{c} \mathbf{a}_{t} \mathbf{u}_{+} \bar{g}_{0}\right)-f\left(\mathbf{k}_{i} \mathbf{a}_{t} \mathbf{u}_{+} \bar{g}_{0}\right)\right|<\varepsilon,
$$

whence

$$
\left|\oint_{\Omega} f\left(\mathbf{c} \mathbf{a}_{t} \mathbf{u}_{+} \bar{g}_{0}\right) d v\left(\mathbf{u}_{+}\right)-\oint_{\Omega} f\left(\mathbf{k}_{i} \mathbf{a}_{t} \mathbf{u}_{+} \bar{g}_{0}\right) d v\left(\mathbf{u}_{+}\right)\right|<\varepsilon,
$$

for any $t \in \mathbb{R}$ and $g_{0} \in G$.

Now

$$
\oint_{\Omega} f\left(\mathbf{k}_{i} \mathbf{a}_{t} \mathbf{u}_{+} \bar{g}_{0}\right) d v\left(\mathbf{u}_{+}\right)=\oint_{\mathbf{k}_{i} \Omega \mathbf{k}_{i}^{-1}} f\left(\mathbf{a}_{t} \mathbf{u}_{+} \mathbf{k}_{i} \bar{g}_{0}\right) d v\left(\mathbf{u}_{+}\right) .
$$

The set $\mathbf{k}_{i} \Omega \mathbf{k}_{i}^{-1}$ also satisfies the hypothesis of Proposition 2.6.5. It follows that there exists $T>0$ such that for any $t \geq T$ and any $i \in\{1, \ldots, m\}$,

$$
\left|\oint_{\mathbf{k}_{i} \Omega \mathbf{k}_{i}^{-1}} f\left(\mathbf{a}_{t} \mathbf{u}_{+} \mathbf{k}_{i} \bar{g}_{0}\right) d v\left(\mathbf{u}_{+}\right)-\oint_{G / \Gamma} f d \mu\right|<\varepsilon .
$$

We conclude that for every $t \geq T$

$$
\left|\oint_{K \Omega} f\left(\mathbf{c a}_{t} \mathbf{u}_{+} \bar{g}_{0}\right) d \nu\left(\mathbf{u}_{+}\right) d \vartheta(\mathbf{c})-\oint_{G / \Gamma} f d \mu\right|<2 \varepsilon
$$

and that

$$
\left|\oint_{\Phi \Omega} f\left(\mathbf{c a}_{t} \mathbf{u}_{+} \bar{g}_{0}\right) d v\left(\mathbf{u}_{+}\right) d \vartheta(\mathbf{c})-\oint_{G / \Gamma} f d \mu\right|<3 \varepsilon .
$$




\subsection{Counting results}

Throughout the whole of this section we work in the following setting. We consider $\Gamma$ an irreducible lattice in $G$ and $\mathcal{V}=X / \Gamma$ the corresponding locally symmetric space. Let $\bar{r}$ be a maximal singular cusp ray in $\mathcal{V}$, let $r$ be a lift of it and let $\xi=r(\infty)$. We denote by $H b_{t}$ the horoball $H b_{t}(r)$ and by $H_{t}$ its boundary horosphere. When $t=0$ we drop the index. Consider $P=P(r), P^{0}=P^{0}(r)$ and $U=U(r)$ the parabolic, horospherical and respectively the unipotent group of $r$. Let $A_{0}=\left(\mathbf{a}_{t}\right)_{t \in \mathbb{R}}$ be the one-dimensional singular torus such that $r(t)=r(0) \mathbf{a}_{t}$ for every $t \geq 0$. Assume that $\mathbf{u} \mapsto a\left(\mathbf{a}_{t}\right)(\mathbf{u})=\mathbf{a}_{-t} \mathbf{u} \mathbf{a}_{t}$ is a dilating homothety on $U$ of factor $e^{\lambda t}$, with $\lambda>0$. We denote the topological dimension of $U$ by $\Delta$.

Notation: For every $y \in X$ we denote by $K(y)$ the maximal compact subgroup of $G$ stabilizing $y$. According to Section 2.4, we can identify $\xi G$ with $\xi K(y)$ and with $K(y)_{\xi} \backslash K(y)$. Every such identification endows $\xi G$ with a natural measure coming from the measure on $K(y)_{\xi} \backslash K(y)$, which we denote by $\theta_{y}$.

Every open set $\Omega$ in $\xi G$ can be identified with an open set in $K(y)_{\xi} \backslash K(y)$. We denote by $\Omega_{K(y)}$ its pre-image in $K(y)$, also open. The set $\Omega_{K(y)}$ is the maximal set in $K(y)$ such that $\Omega=\xi \Omega_{K(y)}$.

Proposition 2.7.1. Let $\mathcal{O}$ be an open set in $\xi G$, let $x$ be a point in $X$ and let $T>0$. For every $k \in \mathbb{N}$, let $N_{x}(k, \mathcal{O})$ be the number of horoballs $H b \gamma, \gamma \in \Gamma$, with basepoint in $\mathcal{O}$ and such that $\operatorname{dist}(x, H b \gamma)$ is in $[k T,(k+1) T)$. For any $T \geq T_{0}(G)$ and any $x \in X$ we have that

$\mathbf{C}_{1} e^{\lambda(k+1) T \Delta} \theta_{x}(\mathcal{O}) \leq N_{x}(k, \mathcal{O}) \leq \mathbf{C}_{2} e^{\lambda(k+1) T \Delta} \theta_{x}(\mathcal{O})$ for every $k \geq k_{0}(x, \mathcal{O}, H b)$,

where $\mathbf{C}_{i}=\mathbf{C}_{i}(G, \Gamma)$ for $i=1,2$.

Proof. We fix an arbitrary point $x$ in $X$ and an open set $\mathcal{O}$ in $\xi G$. We put $K$ for $K(x)$. We also fix a Weyl chamber $W_{0}$ having $r$ as a face and we denote its stabilizer in $G$ by $K_{0}$. Since $P$ acts transitively on $X$ it follows that there exists $p \in P$ such that $r(0) p=x$. Then $W_{0} p$ is a Weyl chamber of vertex $x$, containing $\xi$ in its boundary at infinity, and $W_{0} p \mathcal{O}_{K}$ is the set of Weyl chambers of vertex $x$ containing one of the points in $\mathcal{O}$ in their boundaries at infinity.

The set of Weyl chambers with vertices on $H_{s}$ containing $\xi$ in their boundaries at infinity is $W_{0} \mathbf{a}_{-s} P^{0}$. Consider the horoball $H b \gamma$. It has its basepoint $\xi \gamma$ in $\mathcal{O}$ and it is at distance at least $k T$ and smaller than $(k+1) T$ from $x$ if and only if for some $s \in[k T,(k+1) T), W_{0} \mathbf{a}_{-s} P^{0} \gamma \cap W_{0} p \mathcal{O}_{K} \neq \emptyset$. This is equivalent to $K_{0} \mathbf{a}_{-s} P^{0} \gamma \cap p \mathcal{O}_{K} \neq \emptyset$. Since $K_{0}$ commutes with $A_{0}$ and it is contained in $P^{0}$, we have that $K_{0} \mathbf{a}_{-s} P^{0}=\mathbf{a}_{-s} P^{0}$. Thus, $N_{x}(k, \mathcal{O})$ is the cardinal of the set

$$
\begin{aligned}
& \left\{\gamma \in\left(\Gamma \cap P^{0}\right) \backslash \Gamma ; \exists s \in[k T,(k+1) T) \text { such that } p \mathcal{O}_{K} \cap \mathbf{a}_{-s} P^{0} \gamma \neq \emptyset\right\} \\
& \quad=\left\{\gamma \in \Gamma /\left(\Gamma \cap P^{0}\right) ; \exists s \in[k T,(k+1) T) \text { such that } p \mathcal{O}_{K} \gamma \cap \mathbf{a}_{-s} P^{0} \neq \emptyset\right\} \\
& \quad=\left\{\gamma \in \Gamma ; \exists s \in[k T,(k+1) T) \text { such that } p \mathcal{O}_{K} \gamma \cap \mathbf{a}_{-s} P^{0} /\left(\Gamma \cap P^{0}\right) \neq \emptyset\right\},
\end{aligned}
$$


where in the last set either we may suppose that we are in $G /\left(\Gamma \cap P^{0}\right)$, or we may suppose that we are in $G$, in which case $P^{0} /\left(\Gamma \cap P^{0}\right)$ signifies a fundamental domain of $P^{0}$ with respect to $\Gamma \cap P^{0}$.

Case 1. Suppose that $G$ has real rank at least 2 . Then $\Gamma$ is an arithmetic lattice and $r$ is a maximal singular $\mathbb{Q}$-wall. According to Section 2.3, the horospherical group $P^{0}$ is equal to $U M^{\prime}=M^{\prime} U$, where $U / U \cap \Gamma$ is compact and $M^{\prime} / M^{\prime} \cap \Gamma$ has finite volume. By Section 2.5, $P^{0} /\left(\Gamma \cap P^{0}\right)$ and $P^{0} /(U \cap \Gamma)\left(M^{\prime} \cap \Gamma\right)$ have a common finite covering. Consequently, we can replace in the counting problem above the former by the latter. Also according to Section 2.5 , if $\mathcal{F}$ is a fundamental domain of $M^{\prime}$ with respect to $M^{\prime} \cap \Gamma$, and $\mathcal{D}$ is a fundamental domain of $U$ with respect to $U \cap \Gamma$ then $\mathcal{F} \mathcal{D}$ is a fundamental domain of $P^{0}$ with respect to $(U \cap \Gamma)\left(M^{\prime} \cap \Gamma\right)$. The counting problem above becomes the counting of the number of times when $\operatorname{proj}_{G}\left(\mathbf{a}_{-s} \mathcal{F D}\right)$ intersects $\operatorname{proj}_{G}\left(p \mathcal{O}_{K}\right)$ in $G / \Gamma$, for $s \in[k T,(k+1) T)$.

Notation: For a small positive number $\varepsilon$ we denote by $B_{\varepsilon}$ the open ball $B(x, \varepsilon)$ in $X$.

We also consider $\mathcal{O}^{\varepsilon-} \subset \mathcal{O} \subset \mathcal{O}^{\varepsilon+}$ with $\mathcal{O}^{\varepsilon-}$ and $\mathcal{O}^{\varepsilon+}$ two open subsets of $\xi G$ very near $\mathcal{O}$.

The map

$$
\Pi: K_{0} \backslash G \rightarrow K(r(0)) \backslash G \times P \backslash G \simeq X \times \xi G
$$

defined by the two projections is $C^{\infty}$. Therefore for two open sets $\mathcal{B}$ in $X$ and $\Omega$ in $\xi G, \Pi^{-1}(\mathcal{B} \times \Omega)$ is an open set in $K_{0} \backslash G$. We denote its pre-image in $G$ by $\Omega_{\mathcal{B}}$. The set $\Omega_{\mathcal{B}}$ is the maximal set in $G$ with the property that $W_{0} \Omega_{\mathcal{B}}$ is the set of Weyl chambers with vertices in $\mathcal{B}$ and containing a point from $\Omega$ in their boundary at infinity. A picture of a set $W_{0} \Omega_{\mathcal{B}}$ can be seen in Figure 3, in the particular case when $X$ is the hyperbolic disk $\mathbb{D}^{2}$.

Upper estimate. We want to define a continuous function $f_{1}$ on $G$ taking values in $[0,1]$ and such that $f_{1}$ is 1 on $\mathcal{O}_{B_{\varepsilon}}$ and 0 outside $\mathcal{O}_{B_{2 \varepsilon}}^{\varepsilon+}$. There exists a continuous function $f_{1}^{1}: X \rightarrow[0,1], f_{1}^{1}=1$ on $B_{\varepsilon}$ and $f_{1}^{1}=0$ outside $B_{2 \varepsilon}$. If $\mathcal{O}^{\varepsilon+}$ is well chosen then there exists a continuous function $f_{1}^{2}: \xi G \rightarrow[0,1], f_{1}^{2}=1$ on $\mathcal{O}$ and $f_{1}^{2}=0$ outside $\mathcal{O}^{\varepsilon+}$. The function $f: X \times \xi G \rightarrow[0,1]$ defined by $f(x, \xi g)=f_{1}^{1}(x) f_{1}^{2}(\xi g)$ is 1 on $B_{\varepsilon} \times \mathcal{O}$ and 0 outside $B_{2 \varepsilon} \times \mathcal{O}^{\varepsilon+}$, and by means of it and of the projection $\Pi$ one can obtain the function $f_{1}$.

If $\varepsilon$ is small enough, $f_{1}$ can also be seen as a function in $G / \Gamma$. Note that $f_{1}$ is a bounded function with compact support, hence uniformly continuous. Proposition 2.6.6 applied to $f_{1}$, the semigroup $\left(\mathbf{a}_{-s}\right)_{s \geq 0}, \mathcal{D} \subset U$ and $\mathcal{F} \subset M^{\prime}$ (or their relative interiors), and $g_{0}=e$ gives that

$$
\oint_{\mathcal{F D}} f_{1}\left(\mathbf{a}_{-s} \mathbf{c u} \bar{e}\right) d v(\mathbf{u}) d \vartheta(\mathbf{c}) \rightarrow \oint_{G / \Gamma} f_{1} d \mu \text { when } s \rightarrow \infty,
$$




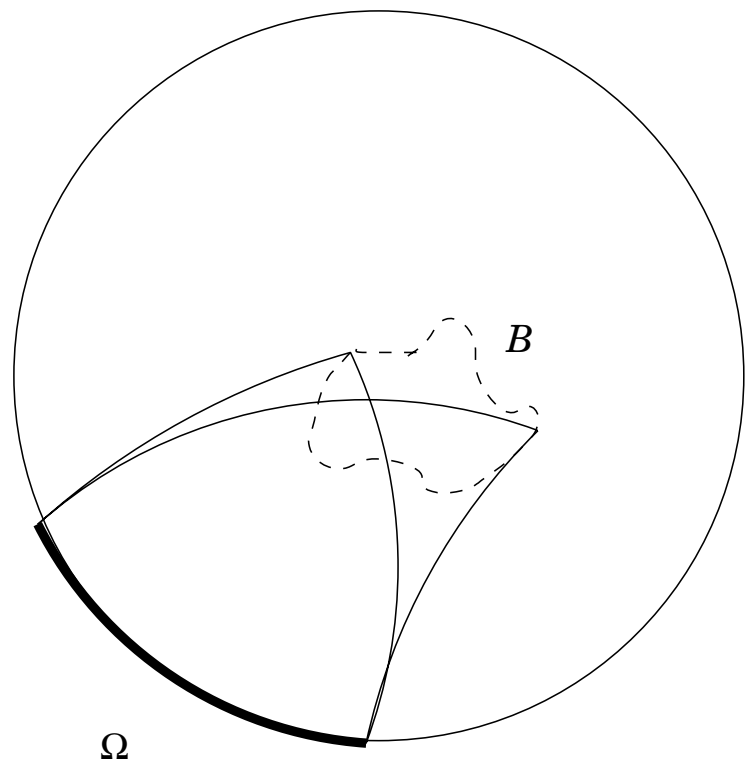

Fig. 3. Example of a set $W_{0} \Omega_{\mathcal{B}}$

where $v$ and $\vartheta$ are the measures induced from the Haar measures on $U$ and $M^{\prime}$, respectively. In particular, for $s \geq s\left(f_{1}\right)$, we have

$$
\oint_{\mathcal{F D}} f_{1}\left(\mathbf{a}_{-s} \mathbf{c u} \bar{e}\right) d v(\mathbf{u}) d \vartheta(\mathbf{c}) \leq 2 \oint_{G / \Gamma} f_{1} d \mu \leq 2 \operatorname{vol}\left(B_{2 \varepsilon}\right)\left[v_{K}\left(\mathcal{O}_{K}\right)+\chi\right],
$$

where $v_{K}$ is the Haar measure on $K$ and $\chi \rightarrow 0$ when $\varepsilon \rightarrow 0$ and $\mathcal{O}^{\varepsilon+}$ converges to $\mathcal{O}$.

This is equivalent to the fact that for $s \geq s\left(f_{1}\right)$,

$$
\oint_{\mathcal{F D}_{s}} f_{1}\left(\mathbf{c u a} \overline{\mathbf{a}}_{-s}\right) d v(\mathbf{u}) d \vartheta(\mathbf{c}) \leq 2 \operatorname{vol}\left(B_{2 \varepsilon}\right)\left[v_{K}\left(\mathcal{O}_{K}\right)+\chi\right],
$$

where $\mathcal{D}_{s}=\mathbf{a}_{-s} \mathcal{D} \mathbf{a}_{s}$. We can rewrite the previous inequality as

$$
\int_{\mathcal{F D}_{s}} f_{1}\left(\mathbf{c u} \overline{\mathbf{a}}_{-s}\right) d v(\mathbf{u}) d \vartheta(\mathbf{c}) \leq 2 \vartheta(\mathcal{F}) e^{\lambda s \Delta} v(\mathcal{D}) \operatorname{vol}\left(B_{2 \varepsilon}\right)\left[v_{K}\left(\mathcal{O}_{K}\right)+\chi\right] .
$$

By integration we obtain

$$
\int_{t}^{t+\tau} \int_{\mathcal{F D}_{s}} f_{1}\left(\mathbf{c u} \overline{\mathbf{a}}_{-s}\right) d v(\mathbf{u}) d \vartheta(\mathbf{c}) d s \leq C_{1} e^{(t+\tau) \lambda \Delta},
$$

where $C_{1}=\frac{2}{\lambda \Delta} \vartheta(\mathcal{F}) \nu(\mathcal{D}) \operatorname{vol}\left(B_{2 \varepsilon}\right)\left[v_{K}\left(\mathcal{O}_{K}\right)+\chi\right]$. 
We have that

$$
\begin{aligned}
\int_{t}^{t+\tau} & \int_{\mathcal{F D}_{s}} f_{1}\left(\mathbf{c u} \overline{\mathbf{a}}_{-s}\right) d v(\mathbf{u}) d \vartheta(\mathbf{c}) d s \\
\geq & \int_{t}^{t+\tau} \int_{\mathcal{F D}_{s}} \mathbf{1}_{\mathcal{O}_{B_{\varepsilon}}}\left(\mathbf{c u} \overline{\mathbf{a}}_{-s}\right) d v(\mathbf{u}) d \vartheta(\mathbf{c}) d s .
\end{aligned}
$$

The second term is the same as

$$
\begin{aligned}
& \int_{t}^{t+\tau} \int_{\mathcal{F} \mathcal{D}_{s}} \mathbf{1}_{\bigsqcup_{\gamma \in \Gamma}} \mathcal{O}_{B_{\varepsilon} \gamma}\left(\mathbf{c u a} \mathbf{a}_{-s}\right) d v(\mathbf{u}) d \vartheta(\mathbf{c}) d s \\
& =\int_{t}^{t+\tau} \int_{\mathcal{F D}} \mathbf{1}_{\sqcup_{\gamma \in \Gamma}} \mathcal{O}_{B_{\varepsilon} \gamma}\left(\mathbf{a}_{-s} \mathbf{c u}\right) d \nu(\mathbf{u}) d \vartheta(\mathbf{c}) e^{\lambda \Delta s} d s \\
& =\sum_{\gamma \in \Gamma} \int_{t}^{t+\tau} \int_{\mathcal{F D}} \mathbf{1}_{\mathcal{O}_{B_{\varepsilon}} \gamma}\left(\mathbf{a}_{-s} \mathbf{c u}\right) d \nu(\mathbf{u}) d \vartheta(\mathbf{c}) e^{\lambda \Delta s} d s .
\end{aligned}
$$

Since $\mathcal{F} \mathcal{D}$ and a fundamental domain $\mathcal{P}$ of $P^{0}$ with respect to $P^{0} \cap \Gamma$ have a common finite covering, the integral above is equivalent (in the sense of the relation $\asymp$ defined in Section 2.1) to the integral

$$
\begin{aligned}
\sum_{\gamma \in \Gamma} & \int_{t}^{t+\tau} \int_{\mathcal{P}} \mathbf{1}_{\mathcal{O}_{B_{\varepsilon}} \gamma}\left(\mathbf{a}_{-s} \mathbf{c u}\right) d \nu(\mathbf{u}) d \vartheta(\mathbf{c}) e^{\lambda \Delta s} d s \\
= & \sum_{\gamma \in \Gamma / P^{0} \cap \Gamma} \sum_{\gamma_{1} \in P^{0} \cap \Gamma} \int_{t}^{t+\tau} \int_{\mathcal{P}} \mathbf{1}_{\mathcal{O}_{B_{\varepsilon}} \gamma \gamma_{1}}\left(\mathbf{a}_{-s} \mathbf{c u}\right) d \nu(\mathbf{u}) d \vartheta(\mathbf{c}) e^{\lambda \Delta s} d s \\
= & \sum_{\gamma \in \Gamma / P^{0} \cap \Gamma} \sum_{\gamma_{1} \in P^{0} \cap \Gamma} \int_{t}^{t+\tau} \int_{\mathcal{P}_{\gamma_{1}}} \mathbf{1}_{\mathcal{O}_{B_{\varepsilon}} \gamma}\left(\mathbf{a}_{-s} \mathbf{c u}\right) d \nu(\mathbf{u}) d \vartheta(\mathbf{c}) e^{\lambda \Delta s} d s \\
= & \sum_{\gamma \in \Gamma / P^{0} \cap \Gamma} \int_{t}^{t+\tau} \sum_{\gamma_{1} \in P^{0} \cap \Gamma} \int_{\mathcal{P}_{\gamma_{1}}} \mathbf{1}_{\mathcal{O}_{B_{\varepsilon}} \gamma}\left(\mathbf{a}_{-s} \mathbf{c u}\right) d \nu(\mathbf{u}) d \vartheta(\mathbf{c}) e^{\lambda \Delta s} d s \\
= & \sum_{\gamma \in \Gamma / P^{0} \cap \Gamma} \int_{t}^{t+\tau} \int_{P^{0}} \mathbf{1}_{\mathcal{O}_{B_{\varepsilon}} \gamma}\left(\mathbf{a}_{-s} \mathbf{c u}\right) d \nu(\mathbf{u}) d \vartheta(\mathbf{c}) e^{\lambda \Delta s} d s .
\end{aligned}
$$

The projection $G \rightarrow K_{0} \backslash G$ sends $K_{0} \mathbf{a}_{-s} P^{0}=\mathbf{a}_{-s} P^{0}$ onto the set of Weyl chambers containing the point $\xi$ in their boundaries at infinity and with vertices on the horosphere $H_{s}$. The image of the set $K_{0} \mathcal{O}_{B_{\varepsilon}} \gamma=\mathcal{O}_{B_{\varepsilon}} \gamma$ is the set of Weyl chambers containing each one point from $\mathcal{O} \gamma$ in their boundaries at infinity, and with vertices in $B_{\varepsilon} \gamma$.

This shows that $\mathbf{1}_{\mathcal{O}_{B_{\varepsilon}} \gamma}$ takes the value 1 on $\mathbf{a}_{-s} P^{0}$ for some $s \in[t, t+\tau]$ if and only if $\xi \in \mathcal{O} \gamma$ and $B_{\varepsilon} \gamma$ intersects the closed strip $H b_{t+\tau} \backslash H b o_{t}$.

Let $\gamma\left(P^{0} \cap \Gamma\right)$ be such that $B_{\varepsilon} \gamma$ is entirely contained in the closed strip $H b_{t+\tau} \backslash H b o_{t}$ and such that $\xi \in \mathcal{O} \gamma$. The first condition is equivalent to 
$d=\operatorname{dist}(x \gamma, H) \in[t+\varepsilon, t+\tau-\varepsilon]$. The second condition is equivalent to the fact that $p \mathcal{O}_{K} \gamma \cap \mathbf{a}_{-d} P^{0} \neq \emptyset$. We can write

$$
\begin{aligned}
\int_{t}^{t+\tau} & \int_{P^{0}} \mathbf{1}_{\mathcal{O}_{B_{\varepsilon}} \gamma}\left(\mathbf{a}_{-s} \mathbf{c u}\right) d v(\mathbf{u}) d \vartheta(\mathbf{c}) e^{\lambda \Delta s} d s \\
\geq & \int_{d-\varepsilon}^{d+\varepsilon} \int_{P^{0}} \mathbf{1}_{\mathcal{O}_{B_{\varepsilon}} \gamma}\left(\mathbf{a}_{-s} \mathbf{c u}\right) d \nu(\mathbf{u}) d \vartheta(\mathbf{c}) e^{\lambda \Delta s} d s
\end{aligned}
$$

The latter term can be rewritten as

$$
\int_{-\varepsilon}^{+\varepsilon} \int_{P^{0}} \mathbf{1}_{\mathcal{O}_{B_{\varepsilon}} \gamma}\left(\mathbf{c u a}_{-(d+s)}\right) d \nu(\mathbf{u}) d \vartheta(\mathbf{c}) d s=\operatorname{vol}\left(B_{\varepsilon}\right) v_{K_{\xi}}\left(K_{\xi}\right) .
$$

Here $v_{K_{\xi}}$ is the measure induced on $K_{\xi}$ by the Haar measure on $P^{0}$.

It follows that the sum in (14) is larger than $N \operatorname{vol}\left(B_{\varepsilon}\right) v_{K_{\xi}}\left(K_{\xi}\right)$, where $N$ is the number of $\gamma \in \Gamma /\left(\Gamma \cap P^{0}\right)$ such that $d=\operatorname{dist}(x \gamma, H) \in[t+\varepsilon, t+\tau-\varepsilon]$ and $p \mathcal{O}_{K} \gamma \cap \mathbf{a}_{-d} P^{0} \neq \emptyset$. If we put $t+\varepsilon=k T$ and $t+\tau-\varepsilon=(k+1) T$ then $N$ is nothing else than $N_{x}(k, \mathcal{O})$. Inequalities (12) and (13) imply that

$$
N_{x}(k, \mathcal{O}) \operatorname{vol}\left(B_{\varepsilon}\right) \leq \mathbf{c}_{2} \operatorname{vol}\left(B_{2 \varepsilon}\right)\left[v_{K}\left(\mathcal{O}_{K}\right)+\chi\right] e^{\lambda \Delta \varepsilon} e^{\lambda \Delta(k+1) T},
$$

where $\mathbf{c}_{2}=\mathbf{c}_{2}(G, \Gamma)$.

For small $\varepsilon$ the ratio $\frac{\operatorname{vol}\left(B_{2 \varepsilon}\right)}{\operatorname{vol}\left(B_{\varepsilon}\right)}$ is bounded from above. Also, if $\varepsilon$ is small enough and $\mathcal{O}^{\varepsilon+}$ is close enough to $\mathcal{O}$, we have that $\chi<v_{K}\left(\mathcal{O}_{K}\right)$ and that $e^{\lambda \Delta \varepsilon}<2$. Thus we obtain

$$
N_{x}(k, \mathcal{O}) \leq \mathbf{C}_{2} v_{K}\left(\mathcal{O}_{K}\right) e^{\lambda \Delta(k+1) T}, \text { for } k \geq k_{0}(\mathcal{O}, x, H b) .
$$

Lower estimate. Consider $f_{2}: G \rightarrow[0,1]$ a continuous function defined to be 1 on $\mathcal{O}_{B_{\varepsilon}}^{\varepsilon-}$ and 0 outside $\mathcal{O}_{B_{2 \varepsilon}}$. Such a function can be constructed for $\mathcal{O}^{\varepsilon-}$ well chosen in a way similar to $f_{1}$. For small $\varepsilon, f_{2}$ can also be seen as a function in $G / \Gamma$. We apply Proposition 2.6.6 as for the upper estimate, but with the function $f_{2}$. We get

$$
\oint_{\mathcal{F} \mathcal{D}} f_{2}\left(\mathbf{a}_{-s} \mathbf{c u} \bar{e}\right) d v(\mathbf{u}) d \vartheta(\mathbf{c}) \rightarrow \oint_{G / \Gamma} f_{2} d \mu,
$$

when $s \rightarrow \infty$. As above, we obtain that for $s \geq s^{\prime}\left(f_{2}\right)$, we have

$$
\oint_{\mathcal{F D}_{s}} f_{2}\left(\mathbf{c u} \overline{\mathbf{a}}_{-s}\right) d v(\mathbf{u}) d \vartheta(\mathbf{c}) \geq \frac{1}{2} \oint_{G / \Gamma} f_{2} d \mu \geq \frac{1}{2} \operatorname{vol}\left(B_{\varepsilon}\right)\left[v_{K}\left(\mathcal{O}_{K}\right)-\chi^{\prime}\right],
$$

where $\chi^{\prime} \rightarrow 0$ when $\varepsilon \rightarrow 0$ and $\mathcal{O}^{\varepsilon-}$ converges to $\mathcal{O}$.

Computations similar to the previous yield

$$
\int_{t}^{t+\tau} \int_{\mathcal{F D}_{s}} f_{2}\left(\mathbf{c u} \overline{\mathbf{a}}_{-s}\right) d \nu(\mathbf{u}) d \vartheta(\mathbf{c}) d s \geq C_{2}^{\prime} e^{t \lambda \Delta},
$$

where $C_{2}^{\prime}=\frac{1}{2} \frac{e^{\tau \lambda \Delta}-1}{\lambda \Delta} \vartheta(\mathcal{F}) v(\mathcal{D}) \operatorname{vol}\left(B_{\varepsilon}\right)\left[v_{K}\left(\mathcal{O}_{K}\right)-\chi^{\prime}\right]$. 
For $\tau$ large enough $e^{\tau \lambda \Delta}-1 \geq \frac{1}{2} e^{\tau \lambda \Delta}$ and we have

$$
\int_{t}^{t+\tau} \int_{\mathcal{F D}_{s}} f_{2}\left(\mathbf{c u} \overline{\mathbf{a}}_{-s}\right) d \nu(\mathbf{u}) d \vartheta(\mathbf{c}) d s \geq C_{2} e^{(t+\tau) \lambda \Delta}
$$

where $C_{2}=\frac{1}{4 \lambda \Delta} \vartheta(\mathcal{F}) v(\mathcal{D}) \operatorname{vol}\left(B_{\varepsilon}\right)\left[v_{K}\left(\mathcal{O}_{K}\right)-\chi^{\prime}\right]$.

Now we write

$$
\begin{aligned}
\int_{t}^{t+\tau} & \int_{\mathcal{F D}_{s}} f_{2}\left(\mathbf{c u} \overline{\mathbf{a}}_{-s}\right) d v(\mathbf{u}) d \vartheta(\mathbf{c}) d s \\
\leq & \int_{t}^{t+\tau} \int_{\mathcal{F D}_{s}} \mathbf{1}_{\mathcal{O}_{B_{2 \varepsilon}}}\left(\mathbf{c u} \overline{\mathbf{a}}_{-s}\right) d v(\mathbf{u}) d \vartheta(\mathbf{c}) d s .
\end{aligned}
$$

The previous argument implies that the second term in (16) is equivalent to the sum

$$
\sum_{\gamma \in \Gamma / P^{0} \cap \Gamma} \int_{t}^{t+\tau} \int_{P^{0}} \mathbf{1}_{\mathcal{O}_{B_{2 \varepsilon}} \gamma}\left(\mathbf{a}_{-s} \mathbf{c u}\right) d \nu(\mathbf{u}) d \vartheta(\mathbf{c}) e^{\lambda \Delta s} d s
$$

The considerations above imply that the sum in (17) is smaller than $N^{\prime} \operatorname{vol}\left(B_{2 \varepsilon}\right)$ $v_{K_{\xi}}\left(K_{\xi}\right)$, where $N^{\prime}$ is the number of $\gamma \in \Gamma / \Gamma \cap P^{0}$ such that $B_{2 \varepsilon} \gamma$ intersects $H b_{t+\tau} \backslash H b o_{t}$ and such that $\xi \in \mathcal{O} \gamma$. These conditions are equivalent to the fact that the distance $d=\operatorname{dist}(x \gamma, H)$ is in $[t-2 \varepsilon, t+\tau+2 \varepsilon]$ and to $p \mathcal{O}_{K} \gamma \cap \mathbf{a}_{-d} P^{0} \neq$ $\emptyset$. Consequently, if we choose $t-2 \varepsilon=k T$ and $t+\tau+2 \varepsilon=(k+1) T$, we obtain $N^{\prime}=N_{x}(k, \mathcal{O})$. Inequalities (15) and (16) imply that

$$
N_{x}(k, \mathcal{O}) \operatorname{vol}\left(B_{2 \varepsilon}\right) \geq \mathbf{c}_{1} \operatorname{vol}\left(B_{\varepsilon}\right)\left[v_{K}\left(\mathcal{O}_{K}\right)-\chi^{\prime}\right] e^{-2 \varepsilon \lambda \Delta} e^{\lambda \Delta(k+1) T},
$$

whence we obtain that for $\varepsilon$ small enough

$$
N_{x}(k, \mathcal{O}) \geq \mathbf{C}_{1} v_{K}\left(\mathcal{O}_{K}\right) e^{\lambda \Delta(k+1) T} \text { for every } k \geq k_{1}(\mathcal{O}, x, H b) .
$$

We now note that $v_{K}\left(\mathcal{O}_{K}\right) \asymp \theta_{x}(\mathcal{O})$.

Case 2. Suppose that $G$ has real rank 1. Then the horospherical group $P^{0}$ is equal to the unipotent group $U$, and we have that $U / U \cap \Gamma$ is compact. One can repeat the same argument as previously, simplified by the fact that there is no central factor $M^{\prime}$, hence $\mathcal{F}$ no longer appears, use Proposition 2.6.5 instead of 2.6.6, and get the same estimate.

Corollary 2.7.2. Let $\rho$ be a geodesic ray such that $\rho(\infty)$ is opposite to $\xi$ and let $U=U(\rho)$. The subset $\xi U$ is open and dense in $\xi G$, it can be identified with $U$ and thus equipped with a measure induced from the measure on $U$, which we denote by $v_{u}$. Let $\Omega$ be a relatively compact open subset of $\xi U$ and let $T>0$. For every open subset $\mathcal{O}$ of $\Omega$, we denote by $N_{r}(k, \mathcal{O})$ the number of horoballs $H b \gamma, \gamma \in \Gamma$, 
with basepoints $\xi \gamma \in \mathcal{O}$ and such that the oriented distance odist $(H b(\rho), H b \gamma)$ is in $[k T,(k+1) T)$. For any $T \geq T_{0}(G, \rho, \Omega)$ we have that

$$
\begin{aligned}
\mathbf{K}_{1} e^{\lambda(k+1) T \Delta} v_{u}(\mathcal{O}) & \leq N_{r}(k, \mathcal{O}) \\
& \leq \mathbf{K}_{2} e^{\lambda(k+1) T \Delta} v_{u}(\mathcal{O}), \text { for every } k \geq k_{0}(\mathcal{O}, \rho, H b),
\end{aligned}
$$

where $\mathbf{K}_{i}=\mathbf{K}_{i}(G, \Gamma, \rho, \Omega)$ for $i=1,2$.

Proof. Let $\mathfrak{G}$ be a geodesic line such that $\mathfrak{G}(+\infty)=\xi, \mathfrak{G}(-\infty)=\rho(\infty)$ and $\mathfrak{G}(0) \in H(\rho)$. Let $\Omega_{U}$ be the relatively compact open subset of $U$ such that $\xi \Omega_{U}=\Omega$. The set $\mathfrak{G}(0) \Omega_{U}$ is a relatively compact subset of $H(\rho)$ of diameter $D$. We choose a point $x$ in it. Let $\widetilde{H b}$ be a horoball with basepoint $\widetilde{\xi}$ in $\Omega$ and such that odist $(H b(\rho), \widetilde{H b})$ is positive and large enough. We have that $\operatorname{dist}(x, \widetilde{H b}) \geq \operatorname{odist}(H b(\rho), \widetilde{H b})$.

Let $\widetilde{u} \in \Omega_{U}$ be such that $\xi \widetilde{u}=\widetilde{\xi}$ and let $\widetilde{\mathfrak{G}}=\mathfrak{G} \widetilde{u}$. Let $t>0$ be such that $\widetilde{\mathfrak{G}}(t)$ is the entrance point of $\widetilde{\mathfrak{G}}$ into $\widetilde{H b}$. Then odist $(H b(\rho), \widetilde{H b})=t$.

We have that $\operatorname{dist}(x, \widetilde{H b}) \leq \operatorname{dist}(x, \widetilde{\mathfrak{G}}(t)) \leq t+\operatorname{dist}(x, \widetilde{\mathfrak{G}}(0))=t+$ $\operatorname{dist}(x, \mathfrak{G}(0) \widetilde{u}) \leq t+D$. Overall we obtain

$$
\operatorname{odist}(H b(\rho), \widetilde{H b}) \leq \operatorname{dist}(x, \widetilde{H b}) \leq \operatorname{odist}(H b(\rho), \widetilde{H b})+D .
$$

This inequality, together with Proposition 2.7.1 and the fact that on $\Omega$ the two measures $v_{u}$ and $\theta_{x}$ are equivalent implies the desired conclusion.

\section{Symmetric spaces of positive definite quadratic forms}

\subsection{The ambient space}

Throughout the paper we shall identify a quadratic form $Q$ on $\mathbb{R}^{s}$ with its matrix $M_{Q}$ in the canonical basis of $\mathbb{R}^{s}$. The matrix of $Q$ in some other basis $\mathcal{B}$ of $\mathbb{R}^{s}$ shall be denoted by $M_{Q}^{\mathcal{B}}$. We shall denote by $b_{Q}$ the bilinear form associated to $Q$.

Let $\mathcal{P}_{s}=S O(s) \backslash S L(s, \mathbb{R})$. This space can be identified with the space of positive definite quadratic forms of determinant one on $\mathbb{R}^{s}$ by associating to each right coset $S O(s) Y$ the quadratic form $Q_{Y}$ whose matrix in the canonical basis is $M_{Y}=Y^{T} \cdot Y$.

We recall that $\mathcal{P}_{s}$ is equipped with a canonical metric defined as follows. Given $Q_{1}, Q_{2} \in \mathcal{P}_{s}$, there exists an orthonormal basis with respect to $Q_{1}$ in which $Q_{2}$ becomes diagonal with coefficients $\lambda_{1}, \ldots, \lambda_{s} \in \mathbb{R}_{+}^{*}$. We define

$$
d\left(Q_{1}, Q_{2}\right)=\sqrt{\sum_{i=1}^{s}\left(\ln \lambda_{i}\right)^{2}} .
$$


Let $\mathcal{Q}_{s}$ be the space of quadratic forms on $\mathbb{R}^{s}$ and $\mathcal{P} \mathcal{Q}_{s}$ the space of positive definite quadratic forms. The group $G L(s, \mathbb{R})$ acts on the right on $\mathcal{Q}_{s}$ by

$$
\Phi: G L(s, \mathbb{R}) \times \mathcal{Q}_{s} \rightarrow \mathcal{Q}_{s}, \Phi(B, M)=B^{T} M B .
$$

This action can be written in terms of quadratic forms as $\Phi(B, Q)=Q[B]=$ $Q \circ B$.

The space $\mathcal{P} \mathcal{Q}_{s}$ is a cone over $\mathcal{P}_{s}$. It is composed of strata of the form

$$
\mathcal{P}_{s}(\delta)=\left\{Q: \mathbb{R}^{s} \rightarrow \mathbb{R} ; Q \text { positive definite quadratic form, det } M_{Q}=\delta\right\},
$$

where $\delta \in \mathbb{R}_{+}^{*}$. We endow each of these strata with a metric defined as in (18). For each $\delta \in \mathbb{R}_{+}^{*}$, any $B \in G L(s, \mathbb{R})$ with $\operatorname{det} B=b$ induces an isometry from $\mathcal{P}_{s}(\delta)$ to $\mathcal{P}_{s}\left(b^{2} \delta\right)$. In particular, each $\mathcal{P}_{s}(\delta)$ is an orbit of $S L(s, \mathbb{R})$.

The subgroup $A=\left\{\operatorname{diag}\left(e^{t_{1}}, e^{t_{2}}, \ldots, e^{t_{s}}\right) ; t_{1}+t_{2}+\cdots+t_{s}=0\right\}$ is a maximal $\mathbb{Q}$-split torus as well as a maximal $\mathbb{R}$-split torus. A $\mathbb{Q}$-Weyl chamber (as well as an $\mathbb{R}$-Weyl chamber $)$ is $\triangleleft A=\left\{\operatorname{diag}\left(e^{t_{1}}, e^{t_{2}}, \ldots, e^{t_{s}}\right) ; t_{1}+t_{2}+\cdots+t_{s}=0, t_{1} \geq\right.$ $\left.t_{2} \geq \cdots \geq t_{s}\right\}$.

Let $Q_{0}$ be the quadratic form of matrix $I d_{s}$. The maximal flat $F_{0}=Q_{0}[A]$ is the set of positive definite quadratic forms $\left\{\operatorname{diag}\left(e^{t_{1}}, e^{t_{2}}, \ldots, e^{t_{s}}\right) ; t_{1}+t_{2}+\cdots+t_{s}=\right.$ $0\}$. The Weyl chamber $W_{0}=Q_{0}[\triangleleft A]$ is the subset of quadratic forms whose matrices moreover satisfy $t_{1} \geq t_{2} \geq \cdots \geq t_{s}$.

The dimension 1 walls (singular rays) of $W_{0}$, parameterized with respect to the arc length, are the sets of quadratic forms

$$
r_{i}=\left\{\operatorname{diag}(\underbrace{e^{\lambda_{i} t}, \ldots, e^{\lambda_{i} t}}_{s-i \text { times }}, \underbrace{e^{-\mu_{i} t}, \ldots e^{-\mu_{i} t}}_{i \text { times }}) ; t \in \mathbb{R}_{+}\right\},
$$

where $\lambda_{i}=\sqrt{\frac{i}{s(s-i)}}$ and $\mu_{i}=\sqrt{\frac{s-i}{s i}}, i \in\{1,2, \ldots s-1\}$.

The parabolic group of $r_{i}$ is the group

$$
\begin{aligned}
P\left(r_{i}\right)=\{ & \left\{\begin{array}{cc}
M_{1} & 0 \\
N & M_{2}
\end{array}\right) \in S L(s, \mathbb{R}) ; \\
& \left.M_{1} \in G L(s-i, \mathbb{R}), M_{2} \in G L(i, \mathbb{R}), N \in M_{i \times(s-i)}(\mathbb{R})\right\} .
\end{aligned}
$$

The horospherical subgroup is

$$
\begin{aligned}
P^{0}\left(r_{i}\right)= & \left\{\left(\begin{array}{cc}
\epsilon M_{1} & 0 \\
N & \epsilon M_{2}
\end{array}\right) ;\right. \\
& \left.M_{1} \in S L(s-i, \mathbb{R}), M_{2} \in S L(i, \mathbb{R}), \epsilon \in\{ \pm 1\}, N \in M_{i \times(s-i)}(\mathbb{R})\right\} .
\end{aligned}
$$

The opposite unipotent group is

$$
U_{+}\left(r_{i}\right)=\left\{\left(\begin{array}{cc}
I d_{s-i} & N \\
0 & I d_{i}
\end{array}\right) ; N \in M_{(s-i) \times i}(\mathbb{R})\right\} .
$$


The boundary at infinity $\partial_{\infty} \mathcal{P}_{s}$ can be identified with the spherical building of flags in $\mathbb{R}^{s}$. Via this identification, $r_{1}(\infty)=\left\langle e_{s}\right\rangle$ and more generally $r_{i}(\infty)$ is the subspace $\left\langle e_{s-i+1}, \ldots, e_{s}\right\rangle$, for $i \in\{1,2, \ldots s-1\}$. The spherical chamber $W_{0}(\infty)$ is identified with the flag $\left\langle e_{s}\right\rangle \subset \cdots \subset\left\langle e_{s-i+1}, \ldots, e_{s}\right\rangle \subset \cdots \subset\left\langle e_{2}, \ldots, e_{s}\right\rangle$.

According to Section 2.4, we can define a projection sl : $\partial_{\infty} \mathcal{P}_{s} \rightarrow W_{0}(\infty)$ and thus define the slope of a point in $\partial_{\infty} \mathcal{P}_{s}$ and of a ray in $\mathcal{P}_{s}$. In particular a maximal singular ray $r$ has slope $r_{i}(\infty)$ if and only if $r(\infty)$ is a linear subspace of dimension $i$.

Given a flag $\mathcal{F}: V_{1} \subset \cdots \subset V_{k}$ in $\mathbb{R}^{s}$ and a matrix $M \in G L(s, \mathbb{R})$ we denote by $M \mathcal{F}$ the flag $M\left(V_{1}\right) \subset \cdots \subset M\left(V_{k}\right)$.

Remark 3.1.1. The isometric action to the right $\Phi$ of $S L(s, \mathbb{R})$ on $\mathcal{P}_{s}$ induces the action to the right $\Phi$ on $\partial_{\infty} \mathcal{P}_{s}$ identified with the spherical building of flags in $\mathbb{R}^{s}$, defined by $\Phi(B, \mathcal{F})=B^{-1} \mathcal{F}$, where $\mathcal{F}$ is an arbitrary flag.

\subsection{The Busemann functions in the ambient space}

By means of Lemma 2.3.1 we can deduce the Busemann function $f_{r_{i}}$.

Lemma 3.2.1. Let $Q$ be a positive definite quadratic form of determinant 1 on $\mathbb{R}^{s}$, let $Q_{i}$ be its restriction to $\left\langle e_{s-i+1}, \ldots, e_{s}\right\rangle$ and let $\operatorname{det} Q_{i}$ be the determinant of $Q_{i}$ in the basis $\left\{e_{s-i+1}, \ldots, e_{s}\right\}$. Then

$$
f_{r_{i}}(Q)=\sqrt{\frac{s}{(s-i) i}} \ln \operatorname{det} Q_{i} .
$$

Proof. According to Lemma 2.3.1, it is enough to prove that the function $\Phi$ : $\mathcal{P}_{s} \rightarrow \mathbb{R}, \Phi(Q)=\sqrt{\frac{s}{(s-i) i}} \ln \operatorname{det} Q_{i}$, is invariant with respect to $P^{0}\left(r_{i}\right)$ and coincides with the Busemann function on the geodesic line $\mathfrak{G}_{i}$ containing $r_{i}$. The second property is obvious.

It suffices to show that the function $\Psi(Q)=\operatorname{det} Q_{i}$ is $P^{0}\left(r_{i}\right)$-invariant. The symmetric matrix $M_{Q}$ of $Q$ can be written as

$$
\begin{aligned}
M_{Q}= & \left(\begin{array}{cc}
E & F \\
F^{T} & H
\end{array}\right), \\
& E \in M_{s-i}(\mathbb{R}), E=E^{T}, H \in M_{i}(\mathbb{R}), H=H^{T}, F \in M_{(s-i) \times i}(\mathbb{R}) .
\end{aligned}
$$

We have $\Psi(Q)=\operatorname{det} H$. Let $B \in P^{0}\left(r_{i}\right)$,

$$
\begin{aligned}
B= & \left(\begin{array}{cc}
\epsilon M_{1} & 0 \\
N & \epsilon M_{2}
\end{array}\right), \\
& M_{1} \in S L(s-i, \mathbb{R}), M_{2} \in S L(i, \mathbb{R}), \in \in\{ \pm 1\}, N \in M_{i \times(s-i)}(\mathbb{R}) .
\end{aligned}
$$

The quadratic form $Q \circ B$ restricted to $\left\langle e_{s-i+1}, \ldots, e_{s}\right\rangle$ has the matrix $M_{2}^{T} H M_{2}$ in the basis $\left\{e_{s-i+1}, \ldots, e_{s}\right\}$. It follows that $\Psi(Q \circ B)=\operatorname{det} M_{2}^{T} H M_{2}=\operatorname{det} H=$ $\Psi(Q)$. 
In particular we have

$$
f_{r_{1}}(Q)=\sqrt{\frac{s}{s-1}} \ln Q\left(e_{s}\right) \text { and } f_{r_{s-1}}(Q)=\sqrt{\frac{s}{s-1}} \ln Q^{*}\left(e_{1}\right),
$$

where $Q^{*}$ is the "dual quadratic form", that is the quadratic form whose matrix in the canonical basis is $M_{Q}^{*}$, if $M_{Q}$ is the matrix of $Q$.

Lemma 3.2.2. Let $d$ be a line in $\mathbb{R}^{s}$ and let $v$ be a non-zero vector on $d$.

(i) The function $f_{v}: \mathcal{P}_{s} \rightarrow \mathbb{R}$,

$$
f_{v}(Q)=\sqrt{\frac{s}{s-1}} \ln Q(v),
$$

is a Busemann function of basepoint $d$.

(ii) Every Busemann function of basepoint $d$ is of the form $f_{w}$, where $w \in d$, $w \neq 0$.

Proof. (i) We can write $v=B e_{s}$ for some $B \in S L(s, \mathbb{R})$. Then $f_{v}(Q)=$ $\sqrt{\frac{s}{s-1}} \ln Q\left(B e_{s}\right)=f_{r_{1}}(\phi(B)(Q))=f_{\phi(B)^{-1} r_{1}}(Q)$. According to Remark 3.1.1, $\phi\left(B^{-1}\right) r_{1}(\infty)=B r_{1}(\infty)=B\left\langle e_{s}\right\rangle=\langle v\rangle$.

(ii) Let $g$ be a Busemann function of basepoint $d$. Then $g-f_{v}$ is a constant function $c$. This implies that $g=f_{v}+c=f_{w}$, where $w=e^{\frac{c}{2} \sqrt{\frac{s-1}{s}}} v$.

A similar argument gives the following.

Lemma 3.2.3. Let $\mathcal{H}$ be a linear hyperplane in $\mathbb{R}^{s}$ and let $v$ be a non-zero vector orthogonal to it.

(i) The function $f_{v}^{*}: \mathcal{P}_{s} \rightarrow \mathbb{R}$,

$$
f_{v}^{*}(Q)=\sqrt{\frac{s}{s-1}} \ln Q^{*}(v)
$$

is a Busemann function of basepoint $\mathcal{H}$.

(ii) Every Busemann function of basepoint $\mathcal{H}$ is of the form $f_{w}^{*}$, where $w \neq 0$ is orthogonal to $\mathcal{H}$.

We have that $f_{r_{1}}=f_{e_{s}}$ and $f_{r_{s-1}}=f_{e_{1}}^{*}$.

Notation: Given a non-zero vector $v \in \mathbb{R}^{n}$ we denote by $H b_{v}^{a}$ and $H_{v}^{a}$ the horoball and horosphere defined respectively by $f_{v} \leq a$ and $f_{v}=a$. We denote by $H b_{v^{*}}^{a}$ and $H_{v^{*}}^{a}$ the horoball and horosphere defined respectively by $f_{v}^{*} \leq a$ and $f_{v}^{*}=a$. 


\subsection{Totally geodesic symmetric subspaces of $\mathcal{P}_{s}$}

For details on the discussion contained in this paragraph, see [Bo, §I.5]. Let $L: \mathbb{R}^{s} \rightarrow \mathbb{R}$ be a non-degenerate quadratic form of signature $(a, b), a+b=s$. Following [Bo, Chapter I, §5], we denote by $\mathcal{P}_{s}(L)$ the set of positive definite quadratic forms $Q$ such that $|L(\bar{x})| \leq Q(\bar{x}), \forall \bar{x} \in \mathbb{R}^{s}$, and such that $Q$ is minimal in the partially ordered set of positive definite quadratic forms, verifying the previous inequality.

Proposition 3.3.1 ([Bo], Chapter I, Proposition 5.2). The following are equivalent:

(i) $Q \in \mathcal{P}_{s}(L)$;

(ii) There exists a basis $\mathcal{B}$ of $\mathbb{R}^{s}$ with respect to which $M_{Q}^{\mathcal{B}}=I d_{s}$ and $M_{L}^{\mathcal{B}}=I_{a, b}$ (with the notation defined in Section 2.1).

Corollary 3.3.2. If $\operatorname{det} M_{L}=\delta$ then $\mathcal{P}_{s}(L) \subset \mathcal{P}_{s}(|\delta|)$.

Proof. Let $Q \in \mathcal{P}_{s}(L)$. By Proposition 3.3.1, (ii), there exists $P \in G L(s, \mathbb{R})$ such that $M_{L}=P^{T} I_{a, b} P$ and $M_{Q}=P^{T} P$.

We consider $\mathcal{P}_{s}(L)$ with the metric induced from $\mathcal{P}_{s}(|\delta|)$.

Remarks 3.3.3. (1) For every $B \in G L(s, \mathbb{R})$ we have $\mathcal{P}_{s}(L[B])=\mathcal{P}_{s}(L)[B]$.

(2) If $L_{1}$ and $L_{2}$ are two non-degenerate quadratic forms of the same signature then $\mathcal{P}_{S}\left(L_{1}\right)$ and $\mathcal{P}_{S}\left(L_{2}\right)$ are isometric.

Proof. Statement (1) is obvious. Statement (2) is a consequence of (1) and of the discussion in Section 3.1.

Remark 3.3.4. If $L$ is a non-degenerate quadratic form of determinant $\delta$ then $\mathcal{P}_{s}(L)$ is a totally geodesic subspace of $\mathcal{P}_{s}(|\delta|)$.

Proof. By the previous remarks it suffices to prove the statement for $\mathcal{P}_{s}\left(I_{a, b}\right) \subset$ $\mathcal{P}_{s}$. The geodesic symmetry of $\mathcal{P}_{s}$ with respect to $I d_{s}$ is $M_{Q} \rightarrow M_{Q}^{-1}$. The fact that $I d_{s} \in \mathcal{P}_{s}\left(I_{a, b}\right)$ and that $\mathcal{P}_{s}\left(I_{a, b}\right)$ is invariant with respect to the previous geodesic symmetry, together with the homogeneity of $\mathcal{P}_{s}\left(I_{a, b}\right)$, imply that it is a totally geodesic subspace of $\mathcal{P}_{s}$.

Notation: For every quadratic form $L: \mathbb{R}^{s} \rightarrow \mathbb{R}$ we denote by Con $_{L}$ the set of vectors $\bar{x}$ in $\mathbb{R}^{s}$ satisfying the equation $L(\bar{x})=0$.

Proposition 3.3.5 ([Mo], §15, §16, [Wi], §4.G). The boundary at infinity, $\partial_{\infty} \mathcal{P}_{s}(L)$, of $\mathcal{P}_{s}(L)$ can be identified with the spherical building of flags of $\mathbb{R}^{s}$ composed of subspaces totally isotropic with respect to $L$. In particular any line in $C_{L} n_{L}$ is a maximal singular point in $\partial_{\infty} \mathcal{P}_{s}(L)$. 
Remark 3.3.6. The action to the right of $S O_{I}(L)$ on $\partial_{\infty} \mathcal{P}_{S}(L)$ seen as a set of flags, which corresponds to the action to the right on $\mathcal{P}_{s}(L)$ as defined in this paper, is the same as the one given in Remark 3.1.1.

We study the geometry of $\mathcal{P}_{s}(L)$ in more detail. By Remarks 3.3.3, it suffices to study $\mathcal{P}_{s}\left(L_{0}\right)$, where

$$
\begin{aligned}
L_{0}= & 2 x_{1} x_{s}+2 x_{2} x_{s-1}+\cdots+2 x_{\ell} x_{s-\ell+1} \\
& +\epsilon\left(x_{\ell+1}^{2}+\cdots+x_{s-\ell}^{2}\right), \ell=\min (a, b), \epsilon \in\{ \pm 1\} .
\end{aligned}
$$

Let $S O_{I}\left(L_{0}\right)$ be the connected component of the identity of the stabilizer of $L_{0}$. A maximal torus in it is [Bo, §11.16]

$$
T=\left\{\operatorname{diag}(e^{-t_{1}}, \ldots, e^{-t_{\ell}}, \underbrace{1, \ldots, 1}_{s-2 \ell}, e^{t_{\ell}}, \ldots, e^{t_{1}}) ;\left(t_{1}, \ldots, t_{\ell}\right) \in \mathbb{R}^{\ell}\right\},
$$

and a Weyl chamber is

$$
W=\left\{\operatorname{diag}(e^{-t_{1}}, \ldots, e^{-t_{\ell}}, \underbrace{1, \ldots, 1}_{s-2 \ell}, e^{t_{\ell}} \ldots e^{t_{1}}) ; t_{1} \geq t_{2} \geq \cdots \geq t_{\ell}\right\} .
$$

Consider the one-parameter group $\mathcal{A}=\left(\mathbf{a}_{t}\right)_{t \in \mathbb{R}}$, with

$$
\mathbf{a}_{t}=\operatorname{diag}\left(e^{-t / 2 \sqrt{2}}, 1, \ldots, 1, e^{t / 2 \sqrt{2}}\right)
$$

and its sub-semigroup $\mathcal{A}^{+}=\left(\mathbf{a}_{t}\right)_{t \geq 0}$. Let $r$ be the geodesic ray defined by $r(t)=$ $\mathbf{a}_{t}^{T} \mathbf{a}_{t}, \forall t \geq 0$. The parabolic group $P=P(r)$ writes as

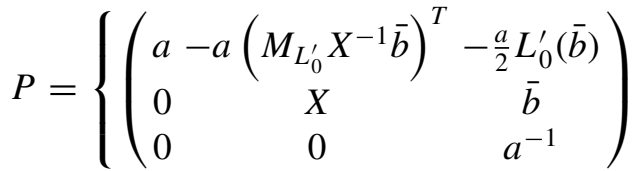

$$
\begin{aligned}
& \left.\in S O_{I}\left(L_{0}\right) ; a \in \mathbb{R}^{*}, X \in S O\left(L_{0}^{\prime}\right), \bar{b} \in \mathbb{R}^{s-2}\right\} \text {, }
\end{aligned}
$$

where $L_{0}^{\prime}: \mathbb{R}^{s-2} \rightarrow \mathbb{R}, L_{0}^{\prime}\left(x_{2}, \ldots, x_{s-1}\right)=2 x_{2} x_{s-1}+\cdots+2 x_{\ell} x_{s-\ell+1}+$ $\epsilon\left(x_{\ell+1}^{2}+\cdots+x_{s-\ell}^{2}\right)$. We note that $P=\left\{g \in S O_{I}\left(L_{0}\right) ; g^{-1}\left(\mathbb{R} e_{1}\right)=\mathbb{R} e_{1}\right\}$. For this reason we also denote it by $P_{e_{1}}$.

The horospherical subgroup of $r$ is

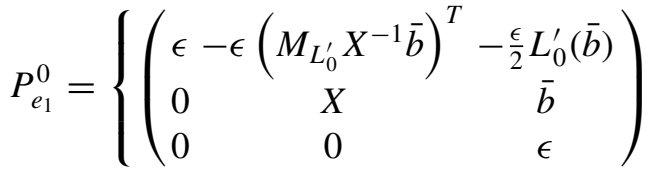

$$
\begin{aligned}
& \left.\in S O_{I}\left(L_{0}\right) ; \epsilon \in\{ \pm 1\}, X \in S O\left(L_{0}^{\prime}\right), \bar{b} \in \mathbb{R}^{s-2}\right\} \text {, }
\end{aligned}
$$


and the unipotent subgroup of $r$ is

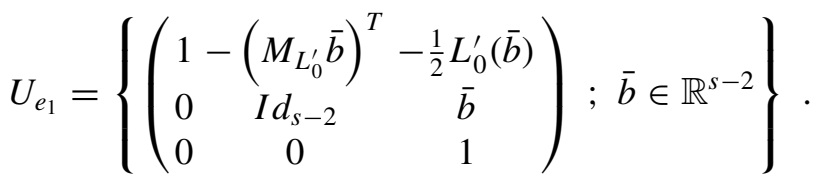

We call a geodesic ray $\rho$ in the orbit $r S O_{I}\left(L_{0}\right)$ maximal singular ray of type $\wp$. The parabolic group corresponding to it, $P(\rho)$, can be written as $\{g \in$ $\left.S O_{I}\left(L_{0}\right) ; g^{-1}(\mathbb{R} v)=\mathbb{R} v\right\}$, where $v \in \operatorname{Con}_{L_{0}} \backslash\{0\}$. It follows that, with the identification of Proposition 3.3.5, $\rho(\infty)=\mathbb{R} v$, that is a point in $\mathbb{P C o n}_{L_{0}}$. Whence the notation $\wp$, coming from "point". We extend in the natural way the notion of maximal singular ray of type $\wp$ to the general case of a non-degenerate quadratic form $L$.

Notation: Let $d$ be a line in $\operatorname{Con}_{L}$. We denote by $P(d)$ the parabolic group

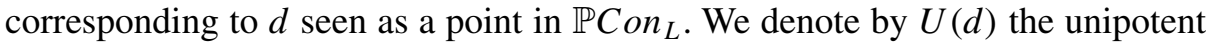
radical of $P(d)$.

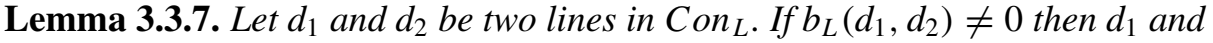
$d_{2}$, seen as maximal singular points in $\partial_{\infty} \mathcal{P}_{s}(L)$, are opposite.

Proof. We show that if $b_{L}\left(d_{1}, d_{2}\right) \neq 0$ then there exists a maximal singular geodesic $\mathfrak{G}$ such that $\mathfrak{G}(-\infty)=d_{1}$ and $\mathfrak{G}(+\infty)=d_{2}$. Let $v \in d_{1}$ and $w \in d_{2}$ be two vectors such that $b_{L}(v, w)=1$. We consider $V=\operatorname{ker} b_{L}(v, \cdot) \cap \operatorname{ker} b_{L}(w, \cdot)$ of dimension $s-2$. By the general theory of non-degenerate quadratic forms (see $[\mathrm{Be}])$ we may choose a basis $\left\{w_{1}, \ldots w_{s-2}\right\}$ of $V$ such that in the coordinates with respect to the basis $\mathcal{B}=\left\{v, w_{1}, \ldots w_{s-2}, w\right\}$ the form $L$ writes as

$$
\begin{aligned}
L= & 2 x_{1} x_{s}+2 x_{2} x_{s-1}+\cdots+2 x_{\ell} x_{s-\ell+1} \\
& +\epsilon\left(x_{\ell+1}^{2}+\cdots+x_{s-\ell}^{2}\right), \ell=\min (a, b), \epsilon \in\{ \pm 1\} .
\end{aligned}
$$

The geodesic $\mathfrak{G}(t)=Q_{t}$ with $M_{Q_{t}}^{\mathcal{B}}=\operatorname{diag}\left(e^{\frac{t}{\sqrt{2}}}, 1, \ldots, 1, e^{-\frac{t}{\sqrt{2}}}\right)$ satisfies $\mathfrak{G}(-\infty)=d_{1}$ and $\mathfrak{G}(+\infty)=d_{2}$.

Remark 3.3.8. Let $d_{1}$ and $d_{2}$ be two lines in $\operatorname{Con}_{L}$ such that $b_{L}\left(d_{1}, d_{2}\right) \neq 0$ and let $\mathcal{H}_{1}$ be the hyperplane defined by $b_{L}\left(d_{1}, \cdot\right)=0$. The map

$$
\begin{aligned}
U\left(d_{1}\right) & \rightarrow \mathbb{P}\left(\text { Con }_{L} \backslash \mathcal{H}_{1}\right) \\
\mathbf{u} & \mapsto \mathbf{u}^{-1} d_{2}
\end{aligned}
$$

is a bijection. 
Proof. By Remarks 3.3.3 and the argument in the proof of Lemma 3.3.7, we may suppose that $L=L_{0}, d_{1}=\mathbb{R} e_{1}, d_{2}=\mathbb{R} e_{s}$ and $\mathcal{H}_{1}=\operatorname{ker} e_{s}^{*}$. It follows that $U\left(d_{1}\right)=U_{e_{1}}$. Consider a unipotent element in $U_{e_{1}}$,

$$
\mathbf{u}(\bar{b})=\left(\begin{array}{ccc}
1-\left(M_{L_{0}^{\prime}} \bar{b}\right)^{T} & -\frac{1}{2} L_{0}^{\prime}(\bar{b}) \\
0 & I d_{s-2} & \bar{b} \\
0 & 0 & 1
\end{array}\right) .
$$

To it corresponds the line $d(\bar{b})$ in $\operatorname{Con}_{L} \backslash \mathcal{H}_{1}$ containing the vector $\left(-\frac{1}{2} L_{0}^{\prime}(\bar{b})\right.$, $-\bar{b}, 1)$ and this establishes a bijection between $U_{e_{1}}$ and the set of lines in $C o n_{L} \backslash \mathcal{H}_{1}$.

Notation: For every line $d \in \mathbb{P}\left(\operatorname{Con}_{L} \backslash \mathcal{H}_{1}\right)$, we denote by $\mathbf{u}_{d}$ the unipotent in $U\left(d_{1}\right)$ corresponding to it by the previous bijection.

\subsection{Horoballs in $\mathcal{P}_{s}(L)$ and counting result}

Lemma 3.4.1. Let $d$ be a line in $\mathrm{Con}_{L}$ and let $v$ be a non-zero vector on $d$.

(i) The function $f_{v}: \mathcal{P}_{s}(L) \rightarrow \mathbb{R}$,

$$
f_{v}(Q)=\sqrt{2} \ln Q(v)
$$

is a Busemann function of basepoint $d$.

(ii) Every Busemann function of basepoint $d$ is of the form $f_{w}$, where $w \in d$, $w \neq 0$.

(iii) Let $v$ be a vector and $r$ a geodesic ray such that $f_{v}=f_{r}$. Then $f_{\lambda v}=$ $f_{r}+2 \sqrt{2} \ln \lambda$.

Proof. (i) Since $L$ is non-degenerate, there exists $w \in \operatorname{Con}_{L}$ such that $b_{L}(v, w)=1$. We proceed as in the proof of Lemma 3.3.7 and consider a basis $\mathcal{B}$ with $v$ and $w$ the first and respectively last vector, with respect to which $L$ can be written as in (22). We consider the geodesic $\mathfrak{G}$ joining $d$ and $\mathbb{R} w, \mathfrak{G}(t)=$ $\left(\Pi^{-1}\right)^{T} \operatorname{diag}\left(e^{\frac{t}{\sqrt{2}}}, 1, \ldots, 1, e^{-\frac{t}{\sqrt{2}}}\right) \Pi^{-1}$, where $\Pi$ is the matrix having the vectors of $\mathcal{B}$ as columns. The geodesic ray corresponding to $d$ is $r(t)=\mathfrak{G}(-t), t \geq 0$, and its horospherical subgroup is $\Pi P_{e_{1}}^{0} \Pi^{-1}$. For every $p \in \Pi P_{e_{1}}^{0} \Pi^{-1}, f_{v}(\bar{Q}[p])=$ $\sqrt{2} \ln Q(p(v))=\sqrt{2} \ln Q(v)$, since $p(v)=v$. Also, $f_{v}(\mathfrak{G}(t))=t$. Lemma 2.3.1 allows to conclude.

(ii) is proved as in Lemma 3.2.2 and (iii) follows immediately from the formula of $f_{v}$. 
Notation: For every $v \in \operatorname{Con}_{L} \backslash\{0\}$ we denote by $H_{v}^{a}, H b_{v}^{a}$ and $H b o_{v}^{a}$ the horosphere defined by $f_{v}=a$, and the horoball and open horoball defined by $f_{v} \leq a$ and $f_{v}<a$, respectively. For $a=0$ we simply write $H_{v}, H b_{v}$ and $H b o_{v}$.

Lemma 3.4.2. Let $v, w$ be two vectors in $C \operatorname{con}_{L}$ such that $b_{L}(v, w) \neq 0$. The oriented distance between the horospheres $H b_{v}$ and $H b_{w}$ is $2 \sqrt{2} \ln \left|b_{L}(v, w)\right|$.

Proof. Let $w_{1}=\frac{1}{\chi} w$ with $\chi=b_{L}(v, w)$. The Busemann function $f_{w}$ is equal to $f_{w_{1}}+2 \sqrt{2} \ln \left|b_{L}(v, w)\right|$. Therefore it suffices to prove the statement of the lemma when $b_{L}(v, w)=1$. By Remark 3.3.3 we may suppose that $L=L_{0}$. Moreover, by Witt Theorem ([Be], [Wi, §4.G]), we may suppose that $v=e_{1}$ and $w=e_{s}$. A geodesic joining $\mathbb{R} e_{1}$ and $\mathbb{R} e_{s}$ is $\mathfrak{G}(t)=\operatorname{diag}\left(e^{-\frac{t}{\sqrt{2}}}, 1, \ldots, 1, e^{\frac{t}{\sqrt{2}}}\right)$. We have that $\mathfrak{G} \cap H_{e_{1}}=\mathfrak{G} \cap H_{e_{s}}=\left\{I d_{s}\right\}$, which finishes the proof.

Corollary 3.4.3. Let $v_{0} \in \operatorname{Con}_{L} \backslash\{0\}$ be fixed and let $\mathcal{H}_{0}$ be the hyperplane defined by $b_{L}\left(v_{0}, \cdot\right)=0$. For every compact set $K$ in $\mathbb{P}\left(\operatorname{Con}_{L} \backslash \mathcal{H}_{0}\right)$, we have that |odist $\left(H b_{v_{0}}, H b_{w}\right)-2 \sqrt{2} \ln \|w\| \mid$ is bounded uniformly in $w \in \mathbb{R} K$.

Corollary 3.4.3 and the counting result Corollary 2.7.2 give the following.

Proposition 3.4.4. Let $\Gamma$ be an irreducible lattice in $S O_{I}(L)$ and let $\bar{r}$ be a maximal singular cusp ray in $\mathcal{P}_{s}(L) / \Gamma$ such that if $r$ is a lift of it in $\mathcal{P}_{s}(L)$, then $r$ is of type $\wp$. Let $r(\infty)=d \in \mathbb{P C o n}{ }_{L}$ and let $v$ be a non-zero vector on $d$. Let $\Omega$ be a relatively compact open subset of $\mathbb{P} C$ Con $_{L}$ such that its closure does not intersect $\mathbb{P}$ ker $b_{L}\left(v_{0}, \cdot\right)$ for some $v_{0} \in C_{\text {Con }} \backslash\{0\}$. Let $a>1$. For every open subset $\mathcal{O}$ of $\Omega$ we denote by $N(k ; \mathcal{O})$ the cardinal of the set of vectors

$$
\left\{v \gamma ; \gamma \in \Gamma, \mathbb{R} v \gamma \in \mathcal{O},\|v \gamma\| \in\left[a^{k}, a^{k+1}\right)\right\} .
$$

For any $a \geq a_{0}(L, \Omega)$ and for any $k \geq k_{0}(\mathcal{O}, \Omega, v)$, we have that

$$
\mathbf{K}_{1} a^{(k+1)(s-2)} v(\mathcal{O}) \leq N(k ; \mathcal{O}) \leq \mathbf{K}_{2} a^{(k+1)(s-2)} v(\mathcal{O}),
$$

where $v$ is the canonical measure on $\mathbb{P} C \operatorname{Con}_{L}$ and $\mathbf{K}_{i}=\mathbf{K}_{i}(L, \Gamma, \Omega)$.

Lemma 3.4.5. Let $d_{0}$ be a fixed line in $\mathrm{Con}_{L}$ and let $\mathcal{H}_{0}$ be the hyperplane defined by $b_{L}\left(d_{0}, \cdot\right)=0$. With the notation following Remark 3.3.8, on every compact subset $\mathcal{K}$ of $\mathbb{P}\left(\operatorname{Con}_{L} \backslash \mathcal{H}_{0}\right)$ the angle between two lines $d_{1}$ and $d_{2}$ in $\mathcal{K}$ is bi-Lipschitz equivalent to the distance between $\mathbf{u}_{d_{1}}$ and $\mathbf{u}_{d_{2}}$ in $U\left(d_{0}\right)$.

Proof. Up to isometry, we can reduce to the case when $L=L_{0}, d_{0}=\mathbb{R} e_{1}$ and $\mathcal{H}_{0}=\operatorname{ker} e_{s}^{*}$. In this case $U\left(d_{0}\right)=U_{e_{1}}$. The Riemannian distance on $U_{e_{1}}$ coming from the Lie group isomorphism

$$
\begin{aligned}
& \mathbb{R}^{s-2} \rightarrow U_{e_{1}} \\
& \bar{b} \mapsto \mathbf{u}(\bar{b})
\end{aligned}
$$

is invariant. With the notation in the proof of Remark 3.3.8, the angle between two lines $d(\bar{b})$ and $d\left(\bar{b}^{\prime}\right)$ is bi-Lipschitz equivalent to $\left\|\bar{b}-\bar{b}^{\prime}\right\|_{e}$, if $\bar{b}$ and $\bar{b}^{\prime}$ are in a compact set of $\mathbb{R}^{s-2}$. 


\subsection{Traces of horoballs on unipotent orbits}

Throughout this section we fix $v_{0}$ a vector in $\operatorname{Con}_{L} \backslash\{0\}$ and a geodesic $\mathfrak{G}$ in $\mathcal{P}_{s}(L)$ with $\mathfrak{G}(-\infty)=d_{0}$, where $d_{0}=\mathbb{R} v_{0}$, and $\mathfrak{G}(0)$ in $H_{v}$. Let $\mathcal{H}_{0}$ be the hyperplane $\operatorname{ker} b_{L}\left(v_{0}, \cdot\right)$, and let $P\left(d_{0}\right)$ be the parabolic group corresponding to $d_{0}$. This group has a Langlands decomposition, $P\left(d_{0}\right)=M A U$ such that $\mathfrak{G}$ is an orbit of $A$. We denote by $r$ the geodesic ray $\left.\mathfrak{G}\right|_{[0,+\infty)}$.

Let $w$ be an arbitrary vector in $\operatorname{Con}_{L} \backslash \mathcal{H}_{0}$ and let $D$ be the oriented distance between the horoballs $H b_{v_{0}}$ and $H b_{w}$. We wish to study the trace of the horoball $H b_{w}$ on $U$ identified with the orbit of $r(t)$ under $U$, that is the set

$$
\operatorname{Tr}_{t}(w)=\left\{\mathbf{u} \in U ; r(t)[\mathbf{u}] \in H b_{w}\right\} .
$$

We put the condition $t=D+\tau, \tau \geq 0$, otherwise $\operatorname{Tr}_{t}(w)$ is empty. We have the following lemma.

Lemma 3.5.1. We consider the unipotent group $U$ endowed with an invariant metric and with the Haar measure $v$. For any vector $w$ in $C n_{L} \backslash \mathcal{H}_{0}$ the following holds. Let $D=\operatorname{odist}\left(H b_{v_{0}}, H b_{w}\right)$.

(1) For any $\tau \geq 0$

$$
\operatorname{Tr}_{D+\tau}(w) \subset B\left(\mathbf{u}_{w}, \kappa_{0} e^{-\frac{D}{2 \sqrt{2}}}\right),
$$

where $\mathbf{u}_{w} \in U$ is such that $\mathfrak{G}\left[\mathbf{u}_{w}\right](+\infty)=\mathbb{R} w$, and $\kappa_{0}$ is a constant depending on $v_{0}$ and on the metric chosen on $U$.

(2) If L has signature $(a, b)$ with $\min (a, b) \geq 2$ then

$$
C_{1} e^{-\frac{D(s-2)+\tau}{2 \sqrt{2}}} \leq v\left(\operatorname{Tr}_{D+\tau}(w)\right) \leq C_{2} e^{-\frac{D(s-2)+\tau}{2 \sqrt{2}}},
$$

where $C_{1}$ and $C_{2}$ are constants depending on $v_{0}$.

Proof. Step 1. First we consider the particular case when $L=L_{0}, v_{0}=e_{1}$ and $\mathfrak{G}_{0}(t)=Q_{t}=\operatorname{diag}\left(e^{\frac{t}{\sqrt{2}}}, 1, \ldots, 1, e^{-\frac{t}{\sqrt{2}}}\right)$. Via the isometry $\bar{b} \mapsto \mathbf{u}(\bar{b})$ we identify $U_{e_{1}}$ to $\mathbb{R}^{s-2}$, its Haar measure $v$ is the Lebesgue measure, and we choose as invariant metric the Euclidean metric. We take the vector $w$ to be $\lambda e_{s}$, with $\lambda=e^{\frac{D}{2 \sqrt{2}}}$, for an arbitrary $D \geq 0$. Let $r(t)=Q_{t}$ for $t \geq 0$.

(1) We have

$$
\begin{aligned}
\operatorname{Tr}_{t}\left(\lambda e_{s}\right) & =\left\{\mathbf{u} \in U_{e_{1}} ; Q_{t}[\mathbf{u}] \in H b_{\lambda e_{s}}\right\}=\left\{\mathbf{u} \in U_{e_{1}} ; Q_{t}[\mathbf{u}]\left(e_{s}\right) \leq \frac{1}{\lambda^{2}}\right\} \\
& =\left\{\mathbf{u}(\bar{b}) \in U_{e_{1}} ; \frac{1}{4} e^{\frac{t}{\sqrt{2}}}\left[L_{0}^{\prime}(\bar{b})\right]^{2}+\|\bar{b}\|_{e}^{2}+e^{-\frac{t}{\sqrt{2}}} \leq e^{-\frac{D}{\sqrt{2}}}\right\} \\
& =\left\{\mathbf{u}(\bar{b}) \in U_{e_{1}} ; \frac{1}{4} e^{\frac{\tau}{\sqrt{2}}}\left[L_{0}^{\prime}\left(e^{\frac{D}{2 \sqrt{2}}} \bar{b}\right)\right]^{2}+\left\|e^{\frac{D}{2 \sqrt{2}} \bar{b}}\right\|_{e}^{2}+e^{-\frac{\tau}{\sqrt{2}}} \leq 1\right\} .
\end{aligned}
$$


We denote by $\mathcal{O}_{\alpha}$ the homothety in $\mathbb{R}^{s-2}$ of center the origin and of factor $\alpha$. We identify $\operatorname{Tr}_{t}\left(\lambda e_{s}\right)$ with $\mathcal{O}_{\eta}\left(\mathcal{M}_{\tau}\right)$, where $\eta=e^{-\frac{D}{2 \sqrt{2}}}$ and

$$
\mathcal{M}_{\tau}=\left\{\bar{b} \in \mathbb{R}^{s-2} ; \frac{1}{4} e^{\frac{\tau}{\sqrt{2}}}\left[L_{0}^{\prime}(\bar{b})\right]^{2}+\|\bar{b}\|_{e}^{2}+e^{-\frac{\tau}{\sqrt{2}}} \leq 1\right\} .
$$

The set $\mathcal{M}_{\tau}$ is quasi-conformal to the set

$$
\mathcal{M}_{\tau}^{\prime}=\left\{\bar{b} \in \mathbb{R}^{s-2} ;\|\bar{b}\|_{e}^{2} \leq 1-e^{-\frac{\tau}{\sqrt{2}}}, \frac{1}{4} e^{\frac{\tau}{\sqrt{2}}}\left[L_{0}^{\prime}(\bar{b})\right]^{2} \leq 1-e^{-\frac{\tau}{\sqrt{2}}}\right\},
$$

in the sense that $\mathcal{O}_{1 / \sqrt{2}}\left(\mathcal{M}_{\tau}^{\prime}\right) \subset \mathcal{M}_{\tau} \subset \mathcal{M}_{\tau}^{\prime}$. We have that $\mathcal{M}_{\tau}^{\prime} \subset B(0,1)$. We conclude that

$$
\operatorname{Tr}_{t}\left(\lambda e_{s}\right) \subset B\left(0, e^{-\frac{D}{2 \sqrt{2}}}\right)
$$

(2) We can rewrite $\mathcal{M}_{\tau}^{\prime}$ as $\mathcal{O}_{\chi}\left(\mathcal{M}_{\tau}^{\prime \prime}\right)$, where $\chi=\sqrt{2}\left(1-e^{-\frac{\tau}{\sqrt{2}}}\right)^{\frac{1}{4}} e^{-\frac{\tau}{4 \sqrt{2}}}$ and

$$
\mathcal{M}_{\tau}^{\prime \prime}=\left\{\bar{b} \in \mathbb{R}^{s-2} ;\left|L_{0}^{\prime}(\bar{b})\right| \leq 1,\|\bar{b}\|_{e} \leq \frac{1}{\sqrt{2}}\left(1-e^{-\frac{\tau}{\sqrt{2}}}\right)^{\frac{1}{4}} e^{\frac{\tau}{4 \sqrt{2}}}\right\} .
$$

Lemma 3.8 from [EMM] implies that

$$
v\left(\mathcal{M}_{\tau}^{\prime \prime}\right) \sim C^{\prime} e^{\frac{\tau(s-4)}{4 \sqrt{2}}} \text { as } \tau \rightarrow \infty,
$$

where $C^{\prime}$ is an absolute constant. Hence

$$
v\left(\mathcal{M}_{\tau}^{\prime}\right) \sim C e^{-\frac{\tau}{2 \sqrt{2}}} \text { as } \tau \rightarrow \infty,
$$

and

$$
C_{1} e^{-\frac{\tau}{2 \sqrt{2}}} \leq v\left(\mathcal{M}_{\tau}\right) \leq C_{2} e^{-\frac{\tau}{2 \sqrt{2}}},
$$

where $C_{1}$ and $C_{2}$ are universal constants. This and the fact that $\operatorname{Tr}_{D+\tau}\left(\lambda e_{s}\right)$ is isometric to $\mathcal{O}_{e^{-D / 2 \sqrt{2}}} \mathcal{M}_{\tau}$ yields the conclusion.

Step 2. We place ourselves in the general case. There exists $B \in G L(n, \mathbb{R})$ such that $\Phi(B)\left(L_{0}\right)=L$. Remark 3.3.3 implies that $\Phi(B)$ is an isometry between $\mathcal{P}_{s}\left(L_{0}\right)$ and $\mathcal{P}_{s}(L)$. The fact that $S O_{I}(L)$ acts transitively on geodesics with both points at infinity lines in $\operatorname{Con}_{L}$ implies that we may suppose that $\Phi(B)\left(\mathfrak{G}_{0}\right)=\mathfrak{G}$. Let $\mathbf{u}_{w} \in U$ be such that $\mathfrak{G}\left[\mathbf{u}_{w}\right](+\infty)=\mathbb{R} w$. Since $\mathbf{u}_{w}$ acts by isometry on $U$ to the right, it suffices to prove the result in the particular case when $\mathbf{u}_{w}=i d$.

We then have that $B^{-1} U_{e_{1}} B=U$ and that $B^{-1} \operatorname{Tr}_{t}\left(\lambda e_{s}\right) B=\operatorname{Tr}_{t}(w)$. The conjugation by $B$ transforms the Haar measure on $U_{e_{1}}$ into the Haar measure on $U$ and the Euclidean metric on $U_{e_{1}}$ into an invariant metric on $U$, bi-Lipschitz equivalent to the one that was chosen. 


\subsection{Quotient spaces, equidistribution of rational vectors}

Let $\mathcal{P}_{s}$ be the ambient symmetric space defined in Section 3.1. Let $\Gamma$ be the lattice $S L(s, \mathbb{Z})$. Its $\mathbb{Q}$-rank $\mathbf{r}$ is equal to the $\mathbb{R}$-rank of $S L(s, \mathbb{R})$ and with $s-1$.

Notation: We denote by $\mathcal{T}_{s}$ the quotient space $\mathcal{P}_{s} / \Gamma$. In accordance with the notation introduced in Section 2.5, we denote by proj the projection of $\mathcal{P}_{s}$ onto $\mathcal{T}_{s}$.

The projection $\bar{W}_{0}=\operatorname{proj}\left(W_{0}\right)$ is isometric to $W_{0}$. Moreover, $\mathcal{T}_{s}$ is at finite Hausdorff distance of $\bar{W}_{0}$. We denote by $\bar{r}_{i}$ the projection of the ray $r_{i}$ defined in (19). According to Remark 2.5.1, (1), and to Lemma 3.2.2, for $a<0$ with $|a|$ large enough, the projection of $H b_{e_{s}}^{a}$ is $H b_{a}\left(\bar{r}_{1}\right)$ and its pre-image is $\bigcup_{v \in \mathcal{P} \mathbb{Z}^{s}} H b_{v}^{a}$. Likewise $H b_{e_{1}^{*}}^{a}$ projects onto $H b_{a}\left(\bar{r}_{s-1}\right)$ and its pre-image is $\bigcup_{v \in \mathcal{P} \mathbb{Z}^{s}} H b_{v^{*}}^{a}$.

Let $L$ be a non-degenerate rational quadratic form on $\mathbb{R}^{s}$. The group $S O_{\mathbb{Z}}(L)$ is a lattice, which we denote by $\Gamma_{L}$. It has $\mathbb{Q}$-rank $\mathbf{r}$ equal to the dimension of the maximal rational linear subspace totally isotropic with respect to $L$ (that is, contained in $\mathrm{Con}_{L}$ ).

Notation: We denote by $\mathcal{V}_{L}$ the quotient space $\mathcal{P}_{S}(L) / \Gamma_{L}$. We denote by $\operatorname{proj}_{L}$ the projection of $\mathcal{P}_{S}(L)$ onto $\mathcal{V}_{L}$.

The manifold $\mathcal{V}_{L}$ is a locally symmetric space of finite volume, at finite Hausdorff distance of a finite union of Euclidean sectors of dimension $\mathbf{r}$, which are projections of $\mathbb{Q}$-Weyl chambers in $\mathcal{P}_{s}(L)$ ([BoS], [Le]). Let $\bar{r}_{1}, \bar{r}_{2}, \ldots, \bar{r}_{k}$ be all the maximal singular cusp rays in $\mathcal{V}_{L}$ such that their lifts $r_{1}, r_{2}, \ldots, r_{k}$ in $\mathcal{P}_{s}(L)$ are of type $\wp$. The set $\left\{\bar{r}_{1}(\infty), \bar{r}_{2}(\infty), \ldots, \bar{r}_{k}(\infty)\right\}$ can be identified with the quotient under the action of $\Gamma_{L}$ of the set of all rational lines in $\operatorname{Con}_{L}$. The latter set can also be seen as the set $\left(\mathcal{P} \mathbb{Z}^{s} \cap \operatorname{Con}_{L}\right) / \pm 1=\mathcal{P} \mathbb{Z}_{+}^{s} \cap \operatorname{Con}_{L}$. Let $r_{i}(\infty)=v_{i} \in \mathcal{P} \mathbb{Z}_{+}^{s} \cap C{ } n_{L}$. By the previous considerations, $\left\{v_{1}, v_{2}, \ldots, v_{k}\right\}$ can also be identified with $\left(\mathcal{P} \mathbb{Z}_{+}^{s} \cap \operatorname{Con}_{L}\right) / \Gamma_{L}$. By Lemma 3.4.1, $f_{r_{i}}=f_{\lambda_{i} v_{i}}$, where $\lambda_{i} \in(0, \infty)$.

According to Remark 2.5.1, (1), if $a<0$ with $|a|$ large enough then for any $i \in\{1,2, \ldots, k\}, \operatorname{proj}_{L}\left(H b_{v_{i}}^{a}\right)$ coincides with $H b_{a_{i}}\left(\bar{r}_{i}\right)$, for some $a_{i}<0$, and the pre-image of it is $H b_{v_{i}}^{a} \Gamma_{L}=\bigcup_{v \in v_{i} \Gamma_{L}} H b_{v}^{a}$. Therefore the projection of $\bigcup_{i=1}^{k} H b_{v_{i}}^{a}$ is $\bigcup_{i=1}^{k} H b_{a_{i}}\left(\bar{r}_{i}\right)$ and it has the pre-image $\bigcup_{v \in \mathcal{P} \mathbb{Z}_{+}^{s} \cap \operatorname{Con}_{L}} H b_{v}^{a}$.

The application of the Proposition 3.4.4 to each of the rays $\bar{r}_{i}$ gives the following.

Proposition 3.6.1. Let $\Omega$ be a relatively compact open subset of $\mathbb{P} C{ }^{2} n_{L}$ such that its closure does not intersect $\mathbb{P} \operatorname{ker} b_{L}\left(v_{0}, \cdot\right)$ for some $v_{0} \in C$ on $_{L} \backslash\{0\}$. Let a $>1$. For every open subset $\mathcal{O}$ of $\Omega$ we denote by $N(k ; \mathcal{O})$ the cardinal of the set of vectors

$$
\left\{v \in \mathcal{P} \mathbb{Z}^{s} \cap \operatorname{Con}_{L} ; \mathbb{R} v \in \mathcal{O},\|v\| \in\left[a^{k}, a^{k+1}\right)\right\}
$$


For any $a \geq a_{0}(L, \Omega)$ we have that $\mathbf{K}_{1} a^{(k+1)(s-2)} v(\mathcal{O}) \leq N(k ; \mathcal{O}) \leq \mathbf{K}_{2} a^{(k+1)(s-2)} v(\mathcal{O})$, for every $k \geq k_{0}(\mathcal{O}, \Omega)$, where $v$ is the canonical measure on $\mathbb{P C o n}{ }_{L}$ and $\mathbf{K}_{i}=\mathbf{K}_{i}(L, \Omega)$.

A consequence of this proposition is Corollary 1.2 in the introduction.

\section{Diophantine approximation on a rational quadric}

\subsection{Some preliminary considerations}

Let $\mathfrak{q}: \mathbb{R}^{n} \rightarrow \mathbb{R}$ be a non-degenerate quadratic form with rational coefficients and let $\mathfrak{Q}_{\mathfrak{q}}$ be the quadric defined by $\mathfrak{q}=1$. Before beginning the proof of Theorem 1.1, we wish to point out that an argument with a projection on a rational hyperplane does not work. This can be illustrated on the example of $\mathfrak{Q}_{\mathfrak{q}}=\mathbb{S}^{n}(0,1) \subset \mathbb{R}^{n+1}$. For simplicity we replace $\mathfrak{Q}_{\mathfrak{q}}$ by $\mathbb{S}^{n}\left(e_{n+1}, 1\right)$, which we denote in what follows by $T \mathbb{S}^{n}$. We recall that the stereographic projection with respect to $2 e_{n+1}$ is

$$
\begin{aligned}
p r: T \mathbb{S}^{n} & \rightarrow \\
\bar{x} & \mapsto \frac{2}{2-x_{n+1}} \bar{x}-\frac{2 x_{n+1}}{2-x_{n+1}} e_{n+1} .
\end{aligned}
$$

Its inverse is

$$
\begin{aligned}
\text { inv }: \mathbb{R}^{n} & \rightarrow \quad T \mathbb{S}^{n} \\
\bar{y} & \mapsto \frac{4}{4+\|\bar{y}\|_{e}^{2}} \bar{y}+\frac{2\|\bar{y}\|_{e}^{2}}{4+\|\bar{y}\|_{e}^{2}} e_{n+1} .
\end{aligned}
$$

We have that

- $\operatorname{pr}\left(\mathcal{S}_{\alpha}\left(T \mathbb{S}^{n}\right)\right) \subset \mathcal{S}_{\alpha-\epsilon}\left(\mathbb{R}^{n}\right)$, for any $\alpha$ and $\epsilon$;

- inv $\left(\mathcal{S}_{\alpha}\left(\mathbb{R}^{n}\right)\right) \subset \mathcal{S}_{\frac{\alpha-1}{2}-\epsilon}\left(T \mathbb{S}^{n}\right)$, for any $\alpha$ and $\epsilon$.

It follows that

$$
\operatorname{inv}\left(\mathcal{S}_{1+2 \alpha+2 \epsilon}\left(\mathbb{R}^{n}\right)\right) \subset \mathcal{S}_{\alpha}\left(T \mathbb{S}^{n}\right) \subset \operatorname{inv}\left(\mathcal{S}_{\alpha-\epsilon}\left(\mathbb{R}^{n}\right)\right) .
$$

On the other hand, by Jarník Theorem, $\operatorname{dim}_{H} \mathcal{S}_{\alpha}\left(\mathbb{R}^{n}\right)=\frac{n+1}{\alpha+1}$ for all $\alpha \geq \frac{1}{n}$. This and relation (25) imply that

$$
\frac{n+1}{2(\alpha+1)} \leq \operatorname{dim}_{H} \mathcal{S}_{\alpha}\left(T \mathbb{S}^{n}\right) \leq \frac{n+1}{\alpha+1} .
$$

For $n=1$ we obtain $\operatorname{dim}_{H} \mathcal{S}_{\alpha}\left(T \mathbb{S}^{1}\right) \geq \frac{1}{1+\alpha}$, which together with the inequality of Melnichuk, $\operatorname{dim}_{H} \mathcal{S}_{\alpha}\left(T \mathbb{S}^{1}\right) \leq \frac{1}{1+\alpha}\left[\mathrm{DD}_{1}\right]$, imply the result of H. Dickinson and M.M. Dodson. For $n>1$ both bounds given by (26) are not sharp.

The first step in the proof of Theorem 1.1 is the following Lemma. 
Lemma 4.1.1. Let $\psi$ be an approximating function such that $\lim _{x \rightarrow \infty} x \psi(x)=0$. Let $\bar{x} \in \mathfrak{Q}_{\mathfrak{q}}$ and let $\frac{1}{q} \bar{p} \in \mathbb{Q}^{n}$ be such that

$$
\left\|\bar{x}-\frac{1}{q} \bar{p}\right\| \leq \frac{\psi(q)}{q} .
$$

If $q$ is large enough then $\frac{1}{q} \bar{p} \in \mathfrak{Q}_{\mathfrak{q}}$.

Proof. We have $\mathfrak{q}(\bar{x})=\sum_{1 \leq i \leq j \leq n} a_{i j} x_{i} x_{j}=1$, where $\bar{x}=\left(x_{1}, \ldots, x_{n}\right)$ and $a_{i j} \in$ $\mathbb{Q}, \forall i, j$. Relation (27) implies that $q x_{i}=p_{i}+\varepsilon_{i}$, where $\bar{p}=\left(p_{1}, p_{2}, \ldots, p_{n}\right)$ and $\varepsilon_{i}=O(\psi(q))$. It follows that $q^{2}=\sum_{1 \leq i \leq j \leq n} a_{i j} q x_{i} q x_{j}=\sum_{1 \leq i \leq j \leq n} a_{i j}\left(p_{i}+\right.$ $\left.\varepsilon_{i}\right)\left(p_{j}+\varepsilon_{j}\right)=\mathfrak{q}(\bar{p})+\sum_{1 \leq i \leq j \leq n} a_{i j}\left(p_{i} \varepsilon_{j}+p_{j} \varepsilon_{i}\right)+\sum_{1 \leq i \leq j \leq n} a_{i j} \varepsilon_{i} \varepsilon_{j}$. We have that $\sum_{1 \leq i \leq j \leq n} a_{i j}\left(p_{i} \varepsilon_{j}+p_{j} \varepsilon_{i}\right)=O(q \psi(q))$ and $\sum_{1 \leq i \leq j \leq n} a_{i j} \varepsilon_{i} \varepsilon_{j}=O\left(\psi(q)^{2}\right)$. Both sums tend to 0 when $q \rightarrow \infty$. Since $\mathfrak{q}$ has rational coefficients, $\mathfrak{q}\left(\mathbb{Z}^{n}\right) \subset \frac{1}{N} \mathbb{Z}$ for some $N \in \mathbb{N}$. It follows that $q^{2}-\mathfrak{q}(\bar{p}) \in \frac{1}{N} \mathbb{Z}$. On the other hand, for $q$ large enough $\left|q^{2}-\mathfrak{q}(\bar{p})\right|<\frac{1}{N}$. We conclude that $\mathfrak{q}\left(\frac{1}{q} \bar{p}\right)=1$ for $q$ large enough.

The previous lemma implies in particular that if $\mathfrak{Q}_{\mathfrak{q}} \cap \mathbb{Q}^{n}=\emptyset$ then $\mathcal{S}_{\psi}\left(\mathfrak{Q}_{\mathfrak{q}}\right)=$ $\emptyset$ for $\psi$ such that $\lim _{x \rightarrow \infty} x \psi(x)=0$. In what follows we work under the hypothesis that $\mathfrak{Q}_{\mathfrak{q}} \cap \mathbb{Q}^{n} \neq \emptyset$.

\subsection{Generalized notion of Hausdorff measure, Hausdorff dimension}

Definitions 4.2.1. (1) A dimension function is a function $\varphi: \mathbb{R}_{+} \rightarrow \mathbb{R}_{+}$which is increasing and continuous and such that $\lim _{x \rightarrow 0} \varphi(x)=0$.

(2) We say that a dimension function $\varphi$ dominates the function $x^{\delta}$ for some $\delta>0$, if

$-x \mapsto x^{-\delta} \varphi(x)$ is a decreasing function;

$-\lim _{x \rightarrow 0} x^{-\delta} \varphi(x)=\infty$.

Let $(\mathcal{M}, d)$ be a metric space and $F$ a non-empty subset of $\mathcal{M}$. For $\epsilon>0$ we call $\epsilon$-cover of $F$ a countable collection of balls $\left\{B_{j}\right\}_{j \in J}$ of radii $r_{j}$ at most $\epsilon$ for every $j \in J$, such that $F \subset \bigcup_{j \in J} B_{j}$. Define

$$
\mathcal{H}_{\epsilon}^{\varphi}(F)=\inf \left\{\sum_{j \in J} \varphi\left(r_{j}\right):\left\{B_{j}\right\}_{j \in J} \text { an } \epsilon-\text { cover of } F\right\} .
$$

The Hausdorff measure of $F$ with respect to the dimension function $\varphi$ is defined by

$$
\mathcal{H}^{\varphi}(F)=\lim _{\epsilon \rightarrow 0} \mathcal{H}_{\epsilon}^{\varphi}(F)=\sup _{\epsilon>0} \mathcal{H}_{\epsilon}^{\varphi}(F) .
$$

Remark 4.2.2. When $\varphi(x)=x^{s}, \mathcal{H}^{\varphi}$ becomes the usual Hausdorff measure $\mathcal{H}^{s}$. Recall that one defines the Hausdorff dimension $\operatorname{dim}_{H} F$ by

$$
\operatorname{dim}_{H} F=\inf \left\{s \geq 0 ; \mathcal{H}^{s}(F)=0\right\}=\sup \left\{s \geq 0 ; \mathcal{H}^{s}(F)=\infty\right\} .
$$




\subsection{Ubiquitous systems}

We need the notion of ubiquitous system as introduced in $\left[\mathrm{BDV}_{2}\right]$, and one of its main properties, which we recall in the sequel. We shall not need the notion in its most general form, as the resonant sets we work with are points. See $\left[\mathrm{BDV}_{2}\right]$ for a more general and detailed presentation, as well as for proofs. We note that in the particular case when the compact metric space considered below $(\mathcal{M}$, dist) is a bounded subset of $\mathbb{R}^{n}$ with the Euclidean metric, and $m$ is the Lebesgue measure, the notion of ubiquitous system and a weaker version of Theorem 4.3.2 were formulated in $\left[\mathrm{DRV}_{2}\right]$ (see also [BD]). In the case when the resonant sets are points this notion coincides with the notion of regular system from [BaS]. Also, a variant of the notion has been defined and used in $[\mathrm{Bu}]$.

Let $(\mathcal{M}$, dist, $m)$ be a compact metric space with a probability measure. Assume that the measure $m$ satisfies the following condition.

(M) There exists $\delta>0$ and $R_{0}>0$ such that for any $x \in \mathcal{M}$ and $R \leq R_{0}$,

$$
a R^{\delta} \leq m(B(x, R)) \leq b R^{\delta} .
$$

The constants $a$ and $b$ are independent of the ball and can be assumed to satisfy

$$
0<a<1<b .
$$

Remark 4.3.1. The condition $(M)$ implies that the Hausdorff dimension $\operatorname{dim}_{H} \mathcal{M}=\delta$.

Let $I$ be an infinite countable family of indices and let $\varpi: I \rightarrow \mathbb{R}_{+}$be a weight function on it. Assume that for every $M>0$, the set $\{i \in I ; \varpi(i) \leq M\}$ is finite. Let $\Re=\left\{p_{i} ; i \in I\right\}$ be a collection of points in $\mathcal{M}$, called resonant points.

Let $\rho: \mathbb{R}_{+} \rightarrow \mathbb{R}_{+}$be a function such that $\lim _{x \rightarrow \infty} \rho(x)=0$. It will be called the ubiquitous function. Let $u=\left(u_{n}\right)_{n \in \mathbb{N}}$ be an increasing sequence of positive real numbers such that $\lim _{n \rightarrow \infty} u_{n}=\infty$. We assume that the function $\rho$ is $u$-regular, that is there exists a constant $0<\lambda<1$ such that for $n \in \mathbb{N}$ sufficiently large,

$$
\rho\left(u_{n+1}\right) \leq \lambda \rho\left(u_{n}\right) .
$$

The pair $(\Re, \varpi)$ is said to be a local m-ubiquitous system relative to $(\rho, u)$ if the following condition is satisfied. There exists $R_{1}>0$ such that an arbitrary ball $B$ in $\mathcal{M}$ of radius $R \leq R_{1}$ satisfies

$$
m\left(B \cap \bigcup_{\varpi(i) \leq u_{n}} B\left(p_{i}, \rho\left(u_{n}\right)\right)\right) \geq \kappa m(B),
$$

for every $n \geq n_{0}(B)$, where $\kappa>0$ is an absolute constant. 
We consider the lim-sup set

$\Lambda(\Re, \psi)=\left\{x \in \mathcal{M} ; \operatorname{dist}\left(x, p_{i}\right)<\psi(\varpi(i))\right.$ for infinitely many $\left.i \in I\right\}$.

Theorem 4.3.2 ([BDV 2$]$, Theorem 3). Let (M, dist, $m)$ be a compact metric space equipped with a measure satisfying the property $(M)$. Let $\rho$ and $u$ be $a$

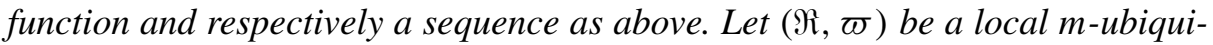
tous system relative to $(\rho, u)$, let $\psi$ be an approximating function and let $\varphi$ be a dimension function dominating $x^{\delta}$.

If $\sum_{n=1}^{\infty} \varphi\left(\psi\left(u_{n}\right)\right) \rho\left(u_{n}\right)^{-\delta}=\infty$ then

$$
\mathcal{H}^{\varphi}(\Lambda(\Re, \psi))=\infty .
$$

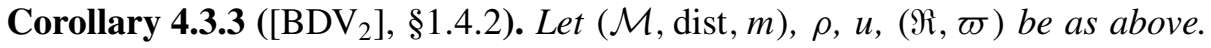
Let $s \in[0, \delta)$.

(1) If $\sum_{n=1}^{\infty} \psi\left(u_{n}\right)^{s} \rho\left(u_{n}\right)^{-\delta}=\infty$ then $\mathcal{H}^{s}(\Lambda(\Re, \psi))=\infty$.

(2) If $\lim _{n \rightarrow \infty} \frac{\psi\left(u_{n}\right)}{\rho\left(u_{n}\right)}=0$ then

$$
\operatorname{dim}_{H} \Lambda(\Re, \psi) \geq \sigma \delta,
$$

where $\sigma=\lim \sup _{n \rightarrow \infty} \frac{\ln \rho\left(u_{n}\right)}{\ln \psi\left(u_{n}\right)}$.

Moreover, if $\lim \inf _{n \rightarrow \infty} \frac{\rho\left(u_{n}\right)}{\psi\left(u_{n}\right)^{\sigma}}<\infty$ then $\mathcal{H}^{\sigma \delta}(\Lambda(\Re, \psi))=\infty$.

\subsection{A geometric definition of $\mathcal{S}_{\psi}\left(\mathfrak{Q}_{\mathfrak{q}}\right)$}

We proceed with the proof of Theorem 1.1. With a countable covering argument, we reduce the problem to the study of $\mathcal{S}_{\psi}(\Omega)$, where $\Omega$ is a relatively compact open subset of $\mathfrak{Q}_{\mathfrak{q}}$ such that its closure does not intersect $T_{\bar{x}_{0}} \mathfrak{Q}_{\mathfrak{q}}$ for some $\bar{x}_{0} \in \mathfrak{Q}_{\mathfrak{q}}$. Let $L_{\mathfrak{q}}: \mathbb{R}^{n+1} \rightarrow \mathbb{R}$ be defined as in the Introduction.

Convention: In what follows we shall drop the index of the form $L_{\mathfrak{q}}$ and we shall adopt for it all the notation introduced in the Sections 3.3 to 3.6.

We note that $\bar{y} \in \mathfrak{Q}_{\mathfrak{q}}$ if and only if $(\bar{y}, 1) \in \operatorname{Con}_{L}$. Thus we may identify $\Omega$ to an open subset of $\mathbb{P} C o n_{L}$, and consider $\mathbb{R} \Omega$. We denote by $v_{0}$ the vector $\left(\bar{x}_{0}, 1\right) \in \operatorname{Con}_{L}$ and by $\mathcal{H}_{0}$ the hyperplane $\operatorname{ker} b_{L}\left(v_{0}, \cdot\right)$. The condition that the closure of $\Omega$ does not intersect $T_{\bar{x}_{0}} \mathfrak{Q}_{\mathfrak{q}}$ is equivalent to the condition that the closure of $\mathbb{R} \Omega$ does not intersect $\mathcal{H}_{0}$. Let $d_{0}=\mathbb{R} v_{0}$. Let $P\left(d_{0}\right)$ be the parabolic subgroup corresponding to $d_{0}$ in $S O_{I}(L)$ and let $M_{0} A_{0} U_{0}$ be a Langlands decomposition of $P\left(d_{0}\right)$. In this case the group $U_{0}$ is Abelian and $A_{0}$ is a maximal singular torus $\left(\mathbf{a}_{t}\right)_{t \in \mathbb{R}}$. We take its parametrization such that $U_{0}=U_{+}\left(A_{0}^{+}\right)$, where $A_{0}^{+}=\left(\mathbf{a}_{t}\right)_{t \geq 0}$. Let $\mathfrak{G}$ be the maximal singular geodesic in $\mathcal{P}_{n+1}(L)$ which is an orbit of $A_{0}$, such that $\mathfrak{G}(0) \in H_{v_{0}}$ and $\mathfrak{G}(-\infty)=d_{0}$. The geodesic ray $r_{0}=\left.\mathfrak{G}\right|_{[0,+\infty)}$ has $U_{+}\left(r_{0}\right)=U_{0}$.

According to Remark 3.3.8, to every $d \in \mathbb{P}\left(\operatorname{Con}_{L} \backslash \mathcal{H}_{0}\right)$ corresponds a unique $\mathbf{u}_{d} \in U_{0}$ such that $d=r_{0}(\infty) \mathbf{u}_{d}$. By means of this, we identify $\Omega$ to a relatively compact open subset of $U_{0}$. 
Convention: We also denote by $\Omega$ the open subset of $\mathbb{P} C{ }^{2} n_{L}$ to which $\Omega \subset \mathfrak{Q}_{\mathfrak{q}}$ is identified via the map $\bar{y} \in \mathfrak{Q}_{\mathfrak{q}} \mapsto(\bar{y}, 1) \in \operatorname{Con}_{L}$. We likewise denote by $\Omega$ the subset in $U_{0}$ to which the previous subset in $\mathbb{P C o n}_{L}$ is identified via the map defined above.

We want to study the set of vectors $\bar{y} \in \Omega$ such that $\left\|\bar{y}-\frac{1}{q} \bar{p}\right\|<\frac{\psi(q)}{q}$ for infinitely many $\frac{1}{q} \bar{p} \in \mathfrak{Q}_{\mathfrak{q}} \cap \mathbb{Q}^{n}$. Without loss of generality we may suppose moreover that $\frac{1}{q} \bar{p} \in \Omega \cap \mathbb{Q}^{n}$.

Let $\bar{y}, \bar{y}^{\prime}$ be points in $\Omega$ and let $d, d^{\prime}$ be the lines in $\operatorname{Con}_{L}$ containing $(\bar{y}, 1)$ and $\left(\bar{y}^{\prime}, 1\right)$, respectively. Lemma 3.4.5 implies that the distance in $U_{0}$ between $\mathbf{u}_{d}$ and $\mathbf{u}_{d^{\prime}}$ is bi-Lipschitz equivalent to the angle between $d$ and $d^{\prime}$, which in its turn is bi-Lipschitz equivalent to $\left\|\bar{y}-\bar{y}^{\prime}\right\|$ for $\bar{y}, \bar{y}^{\prime} \in \Omega$. Corollary 3.4.3 implies that $\|(\bar{p}, q)\|_{e}$ is bi-Lipschitz equivalent to $e^{\text {odist }\left(H b_{v_{0}}, H b_{(\bar{p}, q)}\right) / 2 \sqrt{2}}$. Also, since $\frac{1}{q} \bar{p} \in \Omega$, and $\Omega$ is relatively compact, $\|(\bar{p}, q)\|_{e}$ is bi-Lipschitz equivalent to $q$.

Thus we have

$$
\frac{1}{L} e^{\text {odist }\left(H b_{v_{0}}, H b_{(\bar{p}, q)}\right) / 2 \sqrt{2}} \leq q \leq L e^{\operatorname{odist}\left(H b_{v_{0}}, H b_{(\bar{p}, q)}\right) / 2 \sqrt{2}},
$$

for some $L>1$ depending on $\Omega$.

Let us denote by $\widetilde{\mathcal{S}}_{\Psi}^{0}(\Omega)$ the following subset of $U_{0}$.

$$
\begin{aligned}
& \widetilde{\mathcal{S}}_{\Psi}^{0}(\Omega)=\left\{\mathbf{u} \in \Omega ; \operatorname{dist}_{U_{0}}\left(\mathbf{u}, \mathbf{u}_{(\bar{p}, q)}\right)\right. \\
& \leq \Psi\left(\operatorname{odist}\left(H b_{v_{0}}, H b_{(\bar{p}, q)}\right)\right) \\
& \text { for infinitely many } \\
&\left.(\bar{p}, q) \in \mathcal{P} \mathbb{Z}_{+}^{n+1} \cap \operatorname{Con}_{L}\right\} .
\end{aligned}
$$

The previous considerations imply that for some $L_{1}>1$ we have the inclusions

$$
\begin{aligned}
\widetilde{\mathcal{S}}_{\Psi_{1}}^{0}(\Omega) \subset \mathcal{S}_{\psi}(\Omega) \subset \widetilde{\mathcal{S}}_{\Psi_{2}}^{0}(\Omega), \text { where } \\
\Psi_{1}(x)=\frac{\psi\left(L e^{x / 2 \sqrt{2}}\right)}{L_{1} e^{x / 2 \sqrt{2}}} \text { and } \Psi_{2}(x)=L_{1} \frac{\psi\left(\frac{e^{x / 2 \sqrt{2}}}{L}\right)}{e^{x / 2 \sqrt{2}}} .
\end{aligned}
$$

Therefore we may replace in our study the set $\mathcal{S}_{\psi}(\Omega)$ by the set $\widetilde{\mathcal{S}}_{\Psi}^{0}(\Omega)$ for some approximating function $\Psi$.

Let $\Gamma_{L}, \mathcal{V}_{L}$ and $\left\{v_{1}, v_{2}, \ldots, v_{k}\right\} \subset \mathcal{P} \mathbb{Z}_{+}^{n+1} \cap C_{o n}$ be defined as in Section 3.6. Every $(\bar{p}, q) \in \mathcal{P} \mathbb{Z}_{+}^{n+1} \cap \operatorname{Con}_{L}$ is in the $\Gamma_{L}$-orbit of one of the vectors $\left\{v_{1}, v_{2}, \ldots, v_{k}\right\}$. We fix a vector $v \in\left\{v_{1}, v_{2}, \ldots, v_{k}\right\}$ and we consider the set of vectors $C s p=v \Gamma_{L}$.

We restrict our attention to a subset of $\widetilde{\mathcal{S}}_{\Psi}^{0}(\Omega)$ defined as follows.

$$
\begin{aligned}
\widetilde{\mathcal{S}}_{\Psi}(\Omega)= & \left\{\mathbf{u} \in \Omega ; \operatorname{dist}_{U_{0}}\left(\mathbf{u}, \mathbf{u}_{w}\right)\right. \\
\leq & \Psi\left(\text { odist }\left(H b_{v_{0}}, H b_{w}\right)\right) \\
& \quad \text { for infinitely many } w \in C s p\} .
\end{aligned}
$$


Since the set $\widetilde{\mathcal{S}}_{\Psi}^{0}(\Omega)$ is the union of the sets $\widetilde{\mathcal{S}}_{\Psi}(\Omega)$ defined as in (30) for $i \in\{1,2, \ldots, k\}$, without loss of generality we may replace in our argument $\widetilde{\mathcal{S}}_{\Psi}^{0}(\Omega)$ by $\widetilde{\mathcal{S}}_{\Psi}(\Omega)$.

Notation: In the particular case when $\Psi(x)=e^{-\frac{(1+\alpha) x}{2 \sqrt{2}}}$, with $\alpha>0$, in all the previous notation the index $\Psi$ is replaced by the index $\alpha$.

If the quadratic form $\mathfrak{q}$ is positive definite or of signature $(1, n-1), \mathcal{P}_{s}(L)$ is a model of the $n$-dimensional hyperbolic space. Via the map $\bar{x} \mapsto(\bar{x}, 1), \mathfrak{Q}_{\mathfrak{q}}$ can be identified with $\mathbb{P}\left(\right.$ Con $\left._{L} \backslash \operatorname{ker} e_{n+1}^{*}\right)$. If $\mathfrak{q}$ is positive definite then $\mathbb{P}\left(\right.$ Con $\left._{L} \backslash \operatorname{ker} e_{n+1}^{*}\right)$ $=\mathbb{P}\left(\operatorname{Con}_{L}\right)$, which is the whole boundary at infinity of the hyperbolic space $\mathcal{P}_{s}(L)$. If $\mathfrak{q}$ has signature $(1, n-1)$ then $\mathbb{P}\left(\operatorname{Con}_{L} \backslash \operatorname{ker} e_{n+1}^{*}\right)$ is an open Zariski dense subset of the boundary at infinity of the hyperbolic space. We change the model $\mathcal{P}_{S}(L)$ with the half-space model $\mathbb{H}^{n}$ of the hyperbolic space, and we suppose that $\infty=v_{0}$.

The set $\widetilde{\mathcal{S}}_{\Psi}(\Omega)$ can be identified with the set of points $\vartheta \in \Omega \subset \mathbb{R}^{n-1} \subset \partial_{\infty} \mathbb{H}^{n}$ such that there are infinitely many $\xi \in C s p$ satisfying the inequality

$$
\operatorname{dist}_{U_{0}}\left(\mathbf{u}_{\vartheta}, \mathbf{u}_{\xi}\right) \leq \Psi\left(\operatorname{odist}\left(H b_{\infty}, H b_{\xi}\right)\right) .
$$

The term on the left is $\|\vartheta-\xi\|_{e}$ and the term on the right is, up to some insignificant changes of the function $\Psi, \Psi\left(-\ln h_{\xi}\right)$, where $h_{\xi}$ is the Euclidean height of the horoball $H b_{\xi}$. The set $\widetilde{\mathcal{S}}_{\Psi}(\Omega)$ becomes the set of points $\vartheta \in \Omega$ such that the inequality

$$
\|\vartheta-\xi\|_{e} \leq \Psi\left(-\ln h_{\xi}\right)
$$

has an infinity of solutions $\xi \in C s p$. The equality $\operatorname{dim}_{H} \widetilde{\mathcal{S}}_{\alpha}(\Omega)=\frac{n-1}{1+\alpha}$ is in this case a consequence of a more general result of R. Hill and S. L. Velani [HV]. Moreover, all the results stated in this paper are proved in this particular case in $\left[\mathrm{BDV}_{2}\right]$.

From now on we may therefore suppose that the form $L=L_{\mathfrak{q}}$ is of signature $(a, b)$, with $\min (a, b) \geq 2$.

Notation: We denote $\operatorname{dim} U_{0}$ by $\Delta$.

Since $s=a+b \geq 4$, we have $\Delta=s-2 \geq 2$.

\subsection{Sets of points on a quadric very well approximable by cusp points}

Let $L$ be a non-degenerate quadratic form of signature $(a, b)$, with $\min (a, b) \geq$ 2, $a+b=s$, and let $\Gamma$ be an irreducible lattice in $S O_{I}(L)$. Let $\mathcal{V}$ be the quotient $\mathcal{P}_{s}(L) / \Gamma$ and let proj denote the projection of $\mathcal{P}_{s}(L)$ onto $\mathcal{V}$. As in Section 3.6, we consider the set $\left\{\bar{r}_{1}, \bar{r}_{2}, \ldots, \bar{r}_{k}\right\}$ of all the maximal singular cusp rays in $\mathcal{V}$ such that their lifts $\left\{r_{1}, r_{2}, \ldots, r_{k}\right\}$ in $\mathcal{P}_{s}(L)$ are of type $\wp$. Let $v_{i}$ be a vector 
on the line $r_{i}(\infty), i=1,2, \ldots, k$. The choice of $v_{i}$ shall be made more precise later. We fix a vector $v \in\left\{v_{1}, v_{2}, \ldots, v_{k}\right\}$ and we consider the set of vectors $C s p=v \Gamma$.

Consider the set $\Omega$, the vector $v_{0} \in \operatorname{Con}_{L} \backslash\{0\}$, the geodesic $\mathfrak{G}$, the ray $r_{0}$ and all the notation and properties as in Section 4.4. We define the projection $\pi_{0}: S O_{I}(L) \rightarrow \mathcal{P}_{s}(L), \pi_{0}(g)=\mathfrak{G}(0) g$ and the projection $\pi: S O_{I}(L) / \Gamma \rightarrow \mathcal{V}$ induced by $\pi_{0}$.

Identified with a subset of $U_{0}, \Omega$ can be equipped with the restrictions of a left-invariant metric dist and of the Haar measure $v$. The space $(\Omega$, dist, $v)$ satisfies the condition $(M)$ with $\delta=\Delta$, provided that $\Omega$ is a ball in $U_{0}$. Without loss of generality we assume that $\Omega$ is indeed a ball.

We consider $C s p \cap \mathbb{R} \Omega$ as a countable family of indices and we define

$$
\varpi: C s p \cap \mathbb{R} \Omega \rightarrow \mathbb{R}_{+}, \varpi(w)=\operatorname{odist}\left(H b_{v_{0}}, H b_{w}\right) .
$$

Notation: We denote the oriented distance odist $\left(H b_{v_{0}}, H b_{w}\right)$ by $d_{w}$.

For each $w \in C s p$ let $\mathbf{u}_{w}$ be the unipotent in $U_{0}$ such that $r_{0}\left[\mathbf{u}_{w}\right](\infty)=\mathbb{R} v$. We consider the collection of resonant points $\Re=\left\{\mathbf{u}_{w} ; w \in C \sin \cap \mathbb{R} \Omega\right\}$. Finally we consider the sequence $u=\left(u_{n}\right)_{n \in \mathbb{N}}, u_{n}=n T$, where $T>0$ is large enough, and the ubiquitous function $\rho: \mathbb{R}_{+} \rightarrow \mathbb{R}_{+}, \rho(x)=\varkappa e^{-\frac{x}{2 \sqrt{2}}}$, where $\varkappa$ is a constant to be chosen later. The function $\rho$ is $u$-regular.

Proposition 4.5.1. The pair $(\Re, \varpi)$ is a local $\nu$-ubiquitous system relative to $(\rho, u)$.

Proof. We need to prove that for any ball $B$ in $\Omega$ of radius at most $R_{1}$, for some $R_{1}>0$, we have

$$
v\left(B \cap \bigcup_{w \in C s p \cap \mathbb{R} \Omega, d_{w} \leq n T} B\left(\mathbf{u}_{w}, \varkappa e^{-\frac{n T}{2 \sqrt{2}}}\right)\right) \geq \kappa v(B),
$$

for $n \geq n_{0}(B)$ and $\kappa$ an absolute constant. Without loss of generality we may suppose that $B$ is an open ball in $U_{0}$ which is entirely contained in $\Omega$. We denote by $2 B$ the ball with same center as $B$ and with double radius.

According to Lemma 3.4.1, (iii), multiplying the vector $v$ with a large positive constant $\eta$ means adding to $f_{v}$ a large positive constant $2 \sqrt{2} \ln \eta$. Thus $H b_{\eta v}=$ $H b_{v}^{-2 \sqrt{2}} \ln \eta$. Suppose that $v$ has been re-scaled so that Remark 2.5.1, (1), applies to $H b_{v}$. Let $f: \mathcal{V} \rightarrow \mathbb{R}$ be a $C^{\infty}$-function taking values in $[0,1]$, such that $f=1$ on $\operatorname{proj}\left(H b_{\lambda v}\right)$ for some $\lambda>1$ and close to 1 , and $f=0$ outside $\operatorname{proj}\left(H b_{v}\right)$. According to Proposition 2.6.5, for any $\bar{g}_{0} \in S O_{I}(L) / \Gamma$,

$$
\oint_{B} f \circ \pi\left(\mathbf{a}_{t} \mathbf{u} \bar{g}_{0}\right) d \nu(\mathbf{u}) \rightarrow \oint_{S O_{I}(L) / \Gamma} f \circ \pi d \mu \text { as } t \rightarrow+\infty,
$$


where $\mu$ is the measure on $S O_{I}(L) / \Gamma$ induced by the Haar measure on $S O_{I}(L)$. We take $g_{0}=i d$. The convergence in (31) implies that for $t \geq t_{0}(v, \lambda)$,

$$
\frac{1}{v(B)} \int_{B} \mathbf{1}_{\operatorname{proj}\left(H b_{v}\right)} \circ \pi\left(\mathbf{a}_{t} \mathbf{u} \bar{g}_{0}\right) d v(\mathbf{u}) \geq(1-\epsilon) c(L) V o l_{v},
$$

where $c(L)$ is the total measure of every maximal compact subgroup of $S O_{I}(L)$, $V_{o l}$ is the volume of $\operatorname{proj}\left(H b_{v}\right)$ and $\epsilon$ is a small positive constant such that $\lim _{\lambda \rightarrow 1} \epsilon=0$. We choose and fix $\lambda$ such that $\epsilon=\frac{1}{4}$. The inequality above is equivalent to

$$
v\left(\left\{\mathbf{u} \in B ; \pi_{0}\left(\mathbf{a}_{t} \mathbf{u}\right) \in \bigcup_{w \in C s p} H b_{w}\right\}\right) \geq \frac{3}{4} v(B) c(L) \operatorname{Vol}_{v} .
$$

In (32) it is enough to take the subset of vectors $w$ from $C \operatorname{sp} \cap \mathbb{R} \Omega$ with $d_{w} \leq t$. We note that $\pi_{0}\left(\mathbf{a}_{t} \mathbf{u}\right)=r_{0}(t)[\mathbf{u}]$ for $t \geq 0$. In accordance with the notation in (23), let us denote

$$
T r_{t}(w)=\left\{\mathbf{u} \in U_{0} ; r_{0}(t)[\mathbf{u}] \in H b_{w}\right\} .
$$

We may then write

$$
\left\{\mathbf{u} \in B ; r_{0}(t)[\mathbf{u}] \in \bigcup_{w \in C s p \cap \mathbb{R} \Omega, d_{w} \leq t} H b_{w}\right\}=B \cap \bigcup_{w \in C s p \cap \mathbb{R} \Omega, d_{w} \leq t} \operatorname{Tr}_{t}(w) .
$$

Consider now the subset corresponding to horoballs at distance less than $t-\tau$ for some $\tau>0$ to be chosen later, that is

$$
B \cap \bigcup_{w \in C s p \cap \mathbb{R} \Omega, d_{w}<t-\tau} \operatorname{Tr}_{t}(w) .
$$

Suppose that $t-\tau=k T$ for some $k \in \mathbb{N}$. Then we can write

$$
\bigcup_{w \in C s p \cap \mathbb{R} \Omega, d_{w}<t-\tau} \operatorname{Tr}_{t}(w)=\bigsqcup_{j=-k_{0}}^{k} \bigcup_{w \in C s p \cap \mathbb{R} \Omega, d_{w} \in[(j-1) T, j T)} \operatorname{Tr}_{t}(w) .
$$

We have that

$$
\begin{aligned}
& v\left(B \cap \bigsqcup_{j=-k_{0}}^{k} \bigcup_{w \in C s p \cap \mathbb{R} \Omega, d_{w} \in[(j-1) T, j T)} \operatorname{Tr}_{t}(w)\right) \\
& \quad \leq \sum_{j=-k_{0}}^{k} \sum_{w \in C s p \cap \mathbb{R} \Omega, d_{w} \in[(j-1) T, j T)} v\left(B \cap \operatorname{Tr}_{t}(w)\right) .
\end{aligned}
$$


According to Lemma 3.5.1, (1), $T r_{t}(w) \subset B\left(\mathbf{u}_{w}, \kappa_{0} e^{-\frac{d_{w}}{2 \sqrt{2}}}\right)$, where $\kappa_{0}$ depends on $v_{0}$ and on the metric dist. Hence there exists $J_{0}=J_{0}\left(B, \kappa_{0}\right)$ such that for $j \geq J_{0}$ the intersection $B \cap T r_{t}(w)$ is non-empty only for $w \in C s p$ with $\mathbf{u}_{w} \in 2 B$. To designate the property that $w \in C s p$ with $\mathbf{u}_{w} \in 2 B$ we use the notation $w \in C s p \cap \mathbb{R}(2 B)$. Thus for $j \geq J_{0}$ the sum is taken over the $w \in C \operatorname{sp} \cap \mathbb{R}(2 B), d_{w} \in[(j-1) T, j T)$. Lemma 3.5.1, (2), implies that

$$
\begin{aligned}
& \sum_{w \in C s p \cap \mathbb{R}(2 B), d_{w} \in[(j-1) T, j T)} v\left(B \cap \operatorname{Tr}_{t}(w)\right) \\
& \leq C \sum_{w \in C \operatorname{sp} \cap \mathbb{R}(2 B), d_{w} \in[(j-1) T, j T)} e^{-\frac{(j-1) T \Delta+t-j T}{2 \sqrt{2}}},
\end{aligned}
$$

where $C$ is a constant depending on $v_{0}$. Corollary 2.7.2 now gives that for any $T \geq T_{0}\left(L, v_{0}, \Omega\right)$, the term in (34) is smaller than

$$
\mathbf{K} e^{-\frac{(j-1) T \Delta+t-j T}{2 \sqrt{2}}} e^{\frac{j T \Delta}{2 \sqrt{2}}} v(2 B)=\mathbf{K}^{\prime} e^{\frac{T \Delta+j T-t}{2 \sqrt{2}}} v(B),
$$

for every $j \geq J_{1}$, where $J_{1}=J_{1}\left(B, v, v_{0}\right)$ and $\mathbf{K}^{\prime}=\mathbf{K}^{\prime}\left(L, \Gamma, v_{0}, \Omega\right)$.

Let $J_{2}=\max \left(J_{0}, J_{1}\right)$. The considerations above and Lemma 3.5.1, (2), imply that the measure of the set in (33) is at most

$$
\mathbf{K}^{\prime} e^{\frac{T \Delta-t}{2 \sqrt{2}}} \nu(B) \sum_{j=J_{2}}^{k} e^{\frac{j T}{2 \sqrt{2}}}+C \sum_{j=-k_{0}}^{J_{2}} \sum_{w \in C s p \cap \mathbb{R} \Omega, d_{w} \in[(j-1) T, j T)} e^{-\frac{t+(\Delta-1)(j-1) T}{2 \sqrt{2}}} .
$$

The set of $w \in C \operatorname{sp} \cap \mathbb{R} \Omega$ with $d_{w}<J_{2} T$ is finite, of cardinal $N$. Hence the second term in the sum above is less than

$$
C N \sum_{j=-k_{0}}^{J_{2}} e^{-\frac{t+(\Delta-1)(j-1) T}{2 \sqrt{2}}} \leq C^{\prime} e^{-\frac{t}{2 \sqrt{2}}},
$$

where $C^{\prime}=C^{\prime}\left(v_{0}, v\right.$, dist, $\left.T, B\right)$.

On the whole, for $T \geq 2 \sqrt{2} \ln 2$, the sum in (35) is at most

$$
2 \mathbf{K}^{\prime} e^{\frac{T \Delta-t}{2 \sqrt{2}}} \nu(B) e^{\frac{k T}{2 \sqrt{2}}}+C^{\prime} e^{-\frac{t}{2 \sqrt{2}}} \leq 2 \mathbf{K}^{\prime} e^{\frac{T \Delta-\tau}{2 \sqrt{2}}} \nu(B)+C^{\prime} e^{-\frac{t}{2 \sqrt{2}}},
$$

We choose $\tau$ such that $2 \mathbf{K}^{\prime} e^{\frac{T \Delta-\tau}{2 \sqrt{2}}}=\frac{1}{4} \operatorname{Vol}_{v} c(L)$. Note that it depends on $L, \Gamma, \Omega, v, v_{0}$ and $T$. Then for $t \geq t_{1}$ the measure of the set in (33) is smaller than $\frac{1}{2} \operatorname{Vol}_{v} c(L) v(B)$, where $t_{1}$ depends on $L, v_{0}, v$, dist, $T, B$.

Let $t_{2}=\max \left(t_{0}, t_{1}\right)$. It follows that for $t \geq t_{2}$ the set

$$
B \cap \bigcup_{w \in C s p \cap \mathbb{R} \Omega, t-\tau \leq d_{w} \leq t} \operatorname{Tr}_{t}(w)
$$


has measure at least $\frac{1}{4} \operatorname{Vol}_{v} c(L) v(B)$. By Lemma 3.5.1, (1), this set is included in

$$
\begin{aligned}
& B \cap \bigcup_{w \in C s p \cap \mathbb{R} \Omega, t-\tau \leq d_{w} \leq t} B\left(\mathbf{u}_{w}, \kappa_{0} e^{-\frac{d_{w}}{2 \sqrt{2}}}\right) \\
& \subset B \cap \bigcup_{w \in C s p \cap \mathbb{R} \Omega, t-\tau \leq d_{w} \leq t} B\left(\mathbf{u}_{w}, \kappa_{0} e^{\frac{\tau}{2 \sqrt{2}}} e^{-\frac{t}{2 \sqrt{2}}}\right) .
\end{aligned}
$$

We choose the constant $\varkappa=\kappa_{0} e^{\frac{\tau}{2 \sqrt{2}}}$ and $t=n T$. We have obtained that

$$
v\left(B \cap \bigcup_{w \in C s p \cap \mathbb{R} \Omega, d_{w} \leq n T} B\left(\mathbf{u}_{w}, \varkappa e^{-\frac{n T}{2 \sqrt{2}}}\right)\right) \geq \frac{1}{4} \operatorname{Vol}_{v} c(L) v(B),
$$

for $n \geq n_{0}$, with $n_{0}$ depending on the data of the ubiquitous system and on $B$. This finishes the proof.

For an approximating function $\psi$ we can define the set $\widetilde{\mathcal{S}}_{\psi}(\Omega)=\left\{\mathbf{u} \in \Omega ; \operatorname{dist}_{U_{0}}\left(\mathbf{u}, \mathbf{u}_{w}\right) \leq \psi\left(d_{w}\right)\right.$ for infinitely many $\left.w \in C s p\right\}$. (36)

When $\psi(x)=e^{-\frac{(1+\alpha) x}{2 \sqrt{2}}}$, with $\alpha>0$, we replace the index $\psi$ by the index $\alpha$.

Theorem 4.3.2 implies the following.

Theorem 4.5.2. Let $\varphi$ be a dimension function dominating $x^{\Delta}$. The measure $\mathcal{H}^{\varphi}\left(\widetilde{\mathcal{S}}_{\psi}(\Omega)\right)$ is $+\infty$ if and only if for somelfor every $T>0$ large enough $\sum_{n=1}^{\infty} \varphi(\psi(n T)) e^{\frac{n T \Delta}{2 \sqrt{2}}}=\infty$.

Remark 4.5.3. The significance of the alternative use of the conditions "for some/for every" is the following: the "if" part holds under the weaker condition that the sum is $+\infty$ for some $T>0$ large enough, while in the "only if" part we obtain that the sum is $+\infty$ for every $T>0$ large enough.

Proof. The "if" part follows from Theorem 4.3.2. We show that if for some $T>0$ large enough $\sum_{n=1}^{\infty} \varphi(\psi(n T)) e^{\frac{n T \Delta}{2 \sqrt{2}}}<\infty$ then $\mathcal{H}^{\varphi}\left(\widetilde{\mathcal{S}}_{\psi}(\Omega)\right)<\infty$.

The set $\widetilde{\mathcal{S}}_{\psi}(\Omega)$ is covered by

$$
\bigcup_{w \in C s p \cap \mathbb{R} \mathcal{N}_{\varepsilon}(\Omega)} B\left(\mathbf{u}_{w}, \psi\left(d_{w}\right)\right) .
$$

We have

$$
\begin{aligned}
& \sum_{w \in C s p \cap \mathbb{R} \mathcal{N}_{\varepsilon}(\Omega)} \varphi\left(\psi\left(d_{w}\right)\right)=\sum_{n=1}^{\infty} \sum_{w \in C s p \cap \mathbb{R}_{\varepsilon}(\Omega), d_{w} \in[n T,(n+1) T)} \varphi\left(\psi\left(d_{w}\right)\right) \\
& \ll \sum_{n=1}^{\infty} \varphi(\psi(n T)) e^{\frac{\Delta n T}{2 \sqrt{2}}} .
\end{aligned}
$$

The last inequality follows from Corollary 2.7.2. 
Corollary 4.5.4. (1) Let $s \in[0, \Delta)$. We have $\mathcal{H}^{s}\left(\widetilde{\mathcal{S}}_{\psi}(\Omega)\right)=\infty$ if and only if for somelfor every $T>0$ large enough

$$
\sum_{n=1}^{\infty} \psi(n T)^{s} e^{\frac{n T \Delta}{2 \sqrt{2}}}=\infty .
$$

(2) If for some $T>0$ large enough $\lim _{n \rightarrow \infty} \psi(n T) e^{\frac{n T}{2 \sqrt{2}}}=0$ then

$$
\operatorname{dim}_{H} \widetilde{\mathcal{S}}_{\psi}(\Omega)=\sigma \Delta
$$

where $\sigma=\limsup _{n \rightarrow \infty} \frac{-n T}{2 \sqrt{2} \ln \psi(n T)}$.

Moreover, if $\lim \sup _{n \rightarrow \infty} e^{\frac{n T}{2 \sqrt{2}}} \psi(n T)^{\sigma}>0$ then $\mathcal{H}^{\sigma \Delta}\left(\widetilde{\mathcal{S}}_{\psi}(\Omega)\right)=\infty$.

Proof. Statement (1) follows from Theorem 4.5.2 applied to $\varphi(x)=x^{s}$.

(2) The definition of $\sigma$ implies that for any $\epsilon>0$ the following holds.

(a) For $n$ large enough, $\psi(n T) \leq e^{-\frac{n T}{2 \sqrt{2}(\sigma+\epsilon)}}$;

(b) For infinitely many $n, \psi(n T) \geq e^{-\frac{n T}{2 \sqrt{2}(\sigma-\epsilon)}}$.

According to (a), for every $s>\Delta(\sigma+\epsilon), \sum_{n=1}^{\infty} \psi(n T)^{s} e^{\frac{n T \Delta}{2 \sqrt{2}}} \ll$ $\sum_{n=1}^{\infty} e^{\frac{n T}{2 \sqrt{2}}\left(\Delta-\frac{s}{\sigma+\epsilon}\right)}<+\infty$. Statement (1) implies that $\mathcal{H}^{s}\left(\widetilde{\mathcal{S}}_{\psi}(\Omega)\right)<\infty$.

Property (b) implies that $\psi(n T) e^{\frac{n T}{2 \sqrt{2}(\sigma-\epsilon)}} \geq 1$ for infinitely many $n$. Statement (1) implies that $\mathcal{H}^{\Delta(\sigma-\epsilon)}\left(\widetilde{\mathcal{S}}_{\psi}(\Omega)\right)=\infty$.

We thus obtain that $\Delta(\sigma-\epsilon) \leq \operatorname{dim}_{H} \widetilde{\mathcal{S}}_{\psi}(\Omega) \leq \Delta(\sigma+\epsilon)$, for any $\epsilon>0$, which implies that $\operatorname{dim}_{H} \widetilde{\mathcal{S}}_{\psi}(\Omega)=\Delta \sigma$.

The last statement in (2) follows from (1) applied to $s=\sigma \Delta$.

Corollary 4.5.5. (i) The set $\widetilde{\mathcal{S}}_{\alpha}(\Omega)$ has Hausdorff dimension $d=\frac{\operatorname{dim} U_{0}}{1+\alpha}$ for every $\alpha>0$, and $\mathcal{H}^{d}\left(\widetilde{\mathcal{S}}_{\alpha}(\Omega)\right)=\infty$.

(ii) Both statements hold also for $\mathcal{E}_{\alpha}(\Omega)=\widetilde{\mathcal{S}}_{\alpha}(\Omega) \backslash \bigcup_{\beta>\alpha} \widetilde{\mathcal{S}}_{\beta}(\Omega)$.

Proof. (i) is a consequence of Corollary 4.5.4, (2).

(ii) We can write $\mathcal{E} \widetilde{\mathcal{S}}_{\alpha}(\Omega)=\widetilde{\mathcal{S}}_{\alpha}(\Omega) \backslash \bigcup_{n \in \mathbb{N}} \tilde{\widetilde{\mathcal{S}}}_{\alpha+\frac{1}{n}}(\Omega)$. According to (i) we have, for $d=\frac{\operatorname{dim} U_{0}}{1+\alpha}$, that $\mathcal{H}^{d}\left(\widetilde{\mathcal{S}}_{\alpha}(\Omega)\right)=\infty$ and $\mathcal{H}^{d}\left(\widetilde{\mathcal{S}}_{\alpha+\frac{1}{n}}(\Omega)\right)=0$. Hence $\mathcal{H}^{d}\left(\mathcal{E}_{\alpha}(\Omega)\right)=\infty$.

Remark 4.5.6 (possible generalizations). One might work in the general setting, that is when instead of $\mathcal{P}_{S}(L)$ and $S O_{I}(L)$ there is a general symmetric space of non-compact type $X$ and the connected component of the identity of its group of isometries $G$, and when $\Gamma$ is an irreducible lattice in $G$. One can consider a maximal singular cusp ray $\bar{r}$ in $X / \Gamma$, a lift $r$ in $X, C s p=r(\infty) \Gamma$ and for every $\xi \in C s p$ the horoball $H b_{\xi}$ of basepoint $\xi$ projecting onto $H b(\bar{r})$. 
For an arbitrary ray $\rho$ in the orbit $r G$ and $U_{+}=U_{+}(\rho)$, the set $\rho(\infty) U_{+}$ is open and Zariski dense in $r(\infty) G$ and contains infinitely many $\xi \in C s p$ to which therefore one can associate unipotents $\mathbf{u}_{\xi} \in U_{+}$. A set $\widetilde{\mathcal{S}}_{\psi}$ can be defined as before, that is as the set

$$
\begin{aligned}
\widetilde{\mathcal{S}}_{\psi} & =\left\{\mathbf{u} \in U_{+} ; \operatorname{dist}_{U_{+}}\left(\mathbf{u}, \mathbf{u}_{\xi}\right)\right. \\
& \left.\leq \psi\left(\operatorname{odist}\left(H b_{0}, H b_{\xi}\right)\right) \text { for infinitely many } \xi \in C s p\right\},
\end{aligned}
$$

where $H b_{0}$ is the horoball determined by the ray opposite to $\rho$.

Let $\left(\mathbf{a}_{t}\right)_{t \in \mathbb{R}}$ be the maximal singular torus such that $\rho(t)=\rho(0) \mathbf{a}_{t}$ and suppose that $\mathbf{u} \mapsto \mathbf{a}_{t} \mathbf{u} \mathbf{a}_{-t}$ is a homothety of $U_{+}$of factor $e^{\lambda t}, \lambda>0$. In order to prove that $d=\operatorname{dim}_{H} \widetilde{\mathcal{S}}_{\psi}=\operatorname{dim} U_{+} \sigma$ and $\mathcal{H}^{d}\left(\widetilde{\mathcal{S}}_{\psi}\right)=\infty$, for $\sigma=\limsup _{n \rightarrow \infty} \frac{-\lambda n T}{\ln \psi(n T)}$, the following conditions are sufficient:

- the equidistribution results given in Propositions 2.6.5 and 2.6.6 and the counting result Corollary 2.7.2; these hold in general;

- for $\operatorname{Tr}_{D+\tau}$ as defined in (23), a formula of the measure of the form

$$
v\left(\operatorname{Tr}_{D+\tau}\right)=e^{-D \lambda \operatorname{dim} U_{+}-f(\tau)} \text { with } \lim _{\tau \rightarrow \infty} f(\tau)=\infty ;
$$

- the inclusion $\operatorname{Tr}_{D+\tau} \subset B\left(\mathbf{u}_{\xi}, C e^{-\lambda D}\right)$, with $C$ an absolute constant.

A consequence of Theorem 4.5.2 is the following.

Theorem 4.5.7. Let $\mathfrak{q}: \mathbb{R}^{m} \rightarrow \mathbb{R}$ be a non-degenerate quadratic form with rational coefficients, let $\mathfrak{Q}_{\mathfrak{q}}$ be the quadric defined by $\mathfrak{q}=1$ and let $\psi$ be an approximating function such that $\lim _{x \rightarrow \infty} x \psi(x)=0$. Let $\varphi$ be a dimension function dominating $x^{m-1}$. Then $\mathcal{H}^{\varphi}\left(\mathcal{S}_{\psi}\left(\mathfrak{Q}_{\mathfrak{q}}\right)\right)=\infty$ if and only if for somelfor every $T>0$ large enough $\sum_{n=1}^{\infty} \varphi\left(\frac{\psi\left(e^{n T}\right)}{e^{n T}}\right) e^{n T(m-1)}=\infty$.

Corollaries 4.5.4 and 4.5.5 applied in this setting yield Theorem 1.1.

Remark 4.5.8. The results in Corollary 4.5.5 and in Theorem 1.1 concerning the sets $\mathcal{E} \mathcal{S}_{\alpha}$ of vectors for which the exact order of approximation is known, can be formulated in the more general context of approximating functions. See for example $\left[\mathrm{BDV}_{2}, \S 8.8\right]$ and $\left[\mathrm{BDV}_{3}\right]$ for such results. For the sake of simplicity we have not done it here.

\section{Sets of geodesic rays moving away into the cusp}

\subsection{The case of $\mathcal{P}_{s}(L)$ and of the geodesic rays of type $\wp$}

We consider $L, \Gamma, \mathcal{V}$, proj, $\bar{r}_{i}, r_{i}$ and $v_{i}, i \in\{1,2, \ldots, k\}$, with the same significance as in the beginning of Section 4.5, with the only difference that the condition 
$\min (a, b) \geq 2$ is replaced by $\min (a, b) \geq 1$. Without loss of generality we may suppose that the vector $v_{i}$ is such that $f_{r_{i}}=f_{v_{i}}$. Let $\varrho$ be an arbitrary geodesic ray of type $\wp$ in $\mathcal{P}_{s}(L)$ and let $U_{0}=U_{+}(\varrho)$. We denote $\operatorname{dim} U_{0}$ by $\Delta$.

Consider a function $\phi:[a,+\infty) \rightarrow[b,+\infty), a, b \in \mathbb{R}$, and define the set of unipotents

$$
\begin{aligned}
\mathcal{R}_{\phi}^{i} & =\left\{\mathbf{u} \in U_{0} ;-f_{\bar{r}_{i}}(\operatorname{proj}(\varrho(t) \mathbf{u}))\right. \\
& \geq t-\phi(t) \text { infinitely many times as } t \rightarrow \infty\} .
\end{aligned}
$$

Remark 5.1.1. The maximal possible depth for proj $(\varrho(t) \mathbf{u})$, measured with respect to the ray $\bar{r}_{i}$, is $t+c$, where $c$ is a constant. Such a depth can occur infinitely many times if and only if the ray proj $(\varrho(t) \mathbf{u})$ is asymptotic to $\bar{r}_{i}$ (see Corollary 5.1.5, (c)). Therefore, it makes sense to put $t-\phi(t)$ with $\phi$ a function bounded below near $+\infty$, in the definition of $\mathcal{R}_{\phi}^{i}$.

In the particular case when $t-\phi(t)=\beta t$, with $\beta \in[0,1]$, we replace in our notation in (37) the index $\phi$ by the index $\beta$.

Theorem 5.1.2. Suppose that $\phi$ and $i d-\phi$ are increasing functions, and that $\phi$ is a bijection.

(1) Let $s \in[0, \Delta)$. $\mathcal{H}^{s}\left(\mathcal{R}_{\phi}^{i}\right)=\infty$ if and only if for somelfor every $T>0$ large enough,

$$
\sum_{n=1}^{\infty} e^{\frac{\Delta n T-s \phi^{-1}(n T)}{2 \sqrt{2}}}=\infty .
$$

(2) If for some $T>0$ large enough, $\lim _{n \rightarrow \infty}\left[n T-\phi^{-1}(n T)\right]=-\infty$ then

$$
d=\operatorname{dim}_{H} \mathcal{R}_{\phi}^{i}=\sigma \cdot \Delta \text {, where } \sigma=\limsup _{n \rightarrow \infty} \frac{n T}{\phi^{-1}(n T)} .
$$

If moreover $\lim \sup _{n \rightarrow \infty}\left[n T-\sigma \phi^{-1}(n T)\right]>-\infty$ then $\mathcal{H}^{d}\left(\mathcal{R}_{\phi}^{i}\right)=\infty$.

Proof. We denote by $C s p$ the set $v_{i} \Gamma$. We may restrict our study to a set $\mathcal{R}_{\phi}^{i}(\Omega)=$ $\mathcal{R}_{\phi}^{i} \cap \Omega$, where $\Omega$ is an open relatively compact subset of $U_{0}$. Let $d_{0}$ be the line in $\mathrm{Con}_{L}$ which appears as point at infinity of the geodesic ray $\varrho^{o p}$ opposite to $\varrho$. We choose the vector $v_{0}$ on $d_{0}$ so that $f_{\varrho^{o p}}=f_{v_{0}}$. For every vector $v \in \operatorname{Con}_{L}$ such that $b_{L}\left(v, v_{0}\right) \neq 0$, we denote by $\mathbf{u}_{v}$ the element in $U_{0}$ such that the geodesic ray $\varrho \mathbf{u}_{v}$ has as point at infinity the line $\mathbb{R} v$.

I. We show that $\mathcal{R}_{\phi}^{i}(\Omega) \subset \widetilde{\mathcal{S}}_{\Phi_{1}}(\Omega)$, where $\widetilde{\mathcal{S}}_{\Phi_{1}}(\Omega)$ is defined as in (30) for the approximating function $\Phi_{1}(x)=\kappa_{0} e^{-\frac{\phi^{-1}(x)}{2 \sqrt{2}}}$. Here $\kappa_{0}$ is the constant appearing in the inclusion (24).

Let $\mathbf{u} \in \mathcal{R}_{\phi}^{i}(\Omega)$. It follows that the inequality

$$
f_{w}(\varrho(t) \mathbf{u}) \leq-(t-\phi(t))
$$


has infinitely many solutions $w \in C s p$ and $t \in(0,+\infty)$. Let $w$ and $t$ be two such solutions. Then, with the notation $d_{w}=\operatorname{odist}\left(H b_{v_{0}}, H b_{w}\right)$, we have

$$
d_{w} \leq t-\left|f_{w}(\varrho(t) \mathbf{u})\right| \leq \phi(t) \Rightarrow \phi^{-1}\left(d_{w}\right) \leq t .
$$

The fact that $\varrho(t) \mathbf{u} \in H b_{w}^{-(t-\phi(t))}$ implies by Lemma 3.5.1 that

$$
\operatorname{dist}_{U_{0}}\left(\mathbf{u}, \mathbf{u}_{w}\right) \leq \kappa_{0} e^{-\frac{1}{2 \sqrt{2}}\left(d_{w}+t-\phi(t)\right)} .
$$

The last term of the inequality is by (39) smaller than $\kappa_{0} e^{-\frac{\phi^{-1}\left(d_{w}\right)}{2 \sqrt{2}}}$. We conclude that $\mathcal{R}_{\phi}^{i}(\Omega) \subset \widetilde{\mathcal{S}}_{\Phi_{1}}(\Omega)$.

II. We show that $\mathcal{R}_{\phi}^{i}(\Omega)$ contains $\widetilde{\mathcal{S}}_{\Phi_{2}}(\Omega)$, where $\Phi_{2}(x)=c e^{-\frac{\phi^{-1}(x+1)}{2 \sqrt{2}}}$ for an appropriate constant $c$. Let $\mathbf{u} \in \widetilde{\mathcal{S}}_{\Phi_{2}}(\Omega)$. Let $w \in C s p$ be such that dist $U_{0}\left(\mathbf{u}, \mathbf{u}_{w}\right) \leq$ $c e^{-\frac{\phi^{-1}\left(d_{w}+1\right)}{2 \sqrt{2}}}$. We consider $t=\phi^{-1}\left(d_{w}+1\right)$. We have that

$$
\begin{aligned}
& \left|f_{w}(\varrho(t) \mathbf{u})-f_{w}\left(\varrho(t) \mathbf{u}_{w}\right)\right| \leq \operatorname{dist}\left(\varrho(t) \mathbf{u}, \varrho(t) \mathbf{u}_{w}\right) \\
& \quad \leq e^{\frac{t}{2 \sqrt{2}}} \operatorname{dist}\left(\varrho(0) \mathbf{u}, \varrho(0) \mathbf{u}_{w}\right) \leq 1,
\end{aligned}
$$

if we choose the constant $c$ properly, depending on $\varrho(0)$ and on the metric chosen on $U_{0}$. Since $f_{w}\left(\varrho(t) \mathbf{u}_{w}\right)=d_{w}-t=\phi(t)-1-t$, this implies that $f_{w}(\varrho(t) \mathbf{u}) \leq$ $\phi(t)-t$. We conclude that

$$
-f_{\bar{r}_{i}}(\operatorname{proj}(\varrho(t) \mathbf{u})) \geq t-\phi(t) \text { infinitely many times as } t \rightarrow \infty .
$$

We have obtained that

$$
\widetilde{\mathcal{S}}_{\Phi_{2}}(\Omega) \subset \mathcal{R}_{\phi}^{i}(\Omega) \subset \widetilde{\mathcal{S}}_{\Phi_{1}}(\Omega),
$$

where $\Phi_{1}(x)=\kappa_{0} e^{-\frac{\phi^{-1}(x)}{2 \sqrt{2}}}$ and $\Phi_{2}(x)=c e^{-\frac{\phi^{-1}(x+1)}{2 \sqrt{2}}}$. We apply Corollary 4.5.4 and we obtain the desired conclusion.

Remark 5.1.3. When defining the set $\mathcal{R}_{\phi}^{i}$, one can replace $\phi$ by $\phi_{c}=\phi-c$, where $c$ is a constant, and Theorem 5.1.2 still holds. In order to see this it suffices to show, using the monotonicity of $\phi$, that all the conditions in Theorem 5.1.2 are satisfied by $\phi$ if and only if they are satisfied by $\phi_{c}$. We leave this as an exercise to the reader. See also Remark 5.2.2 where a similar statement is proved in full detail.

Remark 5.1.4. The set $P(\varrho) U_{0}$ is open Zariski dense in $G$, hence the projection of $U_{0}$ is open Zariski dense in $P(\varrho) \backslash G$. We note that $P(\varrho) \backslash G$ is the stratum $\wp$, in the terminology of the Introduction. We also note that if a geodesic ray has a projection on $\mathcal{V}$ moving away into the cusp infinitely many times with depth measured by the function $i d-\phi$ with respect to the ray $\bar{r}_{i}$, then any geodesic ray asymptotic to it has the same property, up to a bounded perturbation of the depth. 
The previous Theorem therefore gives the formula of the Hausdorff dimension of the set of points of type $\wp$ in the boundary at infinity corresponding to rays moving away into the cusp at depth at least $i d-\phi$ with respect to $\bar{r}_{i}$, infinitely many times.

Corollary 5.1.5. Consider the set

$$
\mathcal{R}_{\beta}^{i}=\left\{\mathbf{u} \in U_{0} ;-f_{\bar{r}_{i}}(\operatorname{proj}(\varrho(t) \mathbf{u})) \geq \beta \text { t infinitely many times as } t \rightarrow \infty\right\} .
$$

(a) For any $\beta \in(0,1)$ the set $\mathcal{R}_{\beta}^{i}$ has Hausdorff dimension $d=\Delta(1-\beta)$ and $\mathcal{H}^{d}\left(\mathcal{R}_{\beta}^{i}\right)=\infty$.

(b) Both statements in (a) also hold for the set

$$
\mathcal{E} \mathcal{R}_{\beta}^{i}=\mathcal{R}_{\beta}^{i} \backslash \bigcup_{\beta^{\prime}>\beta} \mathcal{R}_{\beta^{\prime}}^{i}=\left\{\mathbf{u} \in \mathcal{R}_{\beta}^{i} ; \limsup _{t \rightarrow+\infty} \frac{-f_{\bar{r}_{i}}(\operatorname{proj}(\varrho(t) \mathbf{u}))}{t}=\beta\right\} .
$$

(c) The set $\mathcal{R}_{0}^{i}$ coincides with $U_{0}$ and the set $\mathcal{R}_{1}^{i}$ is contained in $\left\{\mathbf{u}_{w} ; w \in C s p\right\}$.

Proof. (a) follows from Theorem 5.1.2, (2), and (b) immediately follows from (a).

(c) For $\beta=0$ it is a consequence of the logarithm law $\left[\mathrm{KM}_{1}\right]$. Suppose that $\beta=1$. Let $\mathbf{u} \in \mathcal{R}_{1}^{i}$. Then for infinitely many $w \in \operatorname{Cs} p$ and $t \in(0,+\infty)$ the following inequality holds:

$$
f_{w}(\varrho(t) \mathbf{u}) \leq-t .
$$

As in (39) we obtain that for every such $w$ we have $d_{w} \leq 0$. The inclusion $\varrho(t) \mathbf{u} \in H b_{w}^{-t}$ implies by Lemma 3.5.1 that

$$
\operatorname{dist}_{U_{0}}\left(\mathbf{u}, \mathbf{u}_{w}\right) \leq \kappa_{0} e^{-\frac{1}{2 \sqrt{2}}\left(d_{w}+t\right)} .
$$

Thus for $t$ large enough we may suppose that the corresponding $w \in C s p$ satisfies $\mathbf{u}_{w} \in B(\mathbf{u}, 1)$. On the other hand, the number of $w \in C s p$ with $\mathbf{u}_{w} \in B(\mathbf{u}, 1)$ and $d_{w} \leq 0$ is finite. Hence, by eventually taking a subsequence we may suppose that $w$ is fixed. By letting $t \rightarrow \infty$ in (41) we obtain that $\mathbf{u}=\mathbf{u}_{w}$. Thus $\mathcal{R}_{1}^{i} \subset\left\{\mathbf{u}_{w} ; w \in C s p\right\}$.

Remark 5.1.6. Both Theorem 5.1.2 and Corollary 5.1.5 follow from Corollary 4.5.4 and inclusion (24). Consequently the conditions in Remark 4.5.6 are sufficient also for the generalization of these two results. 
5.2. The symmetric space $\mathcal{P}_{n+1}$ and the rays of slope $r_{i}(\infty), i=1, n$

Consider the symmetric space $\mathcal{P}_{n+1}$, with group of isometries $S L(n+1, \mathbb{R})$, and the lattice $S L(n+1, \mathbb{Z})$. Let $\mathcal{T}_{n+1}=\mathcal{P}_{n+1} / S L(n+1, \mathbb{Z})$ and let proj be the projection of $\mathcal{P}_{n+1}$ onto $\mathcal{T}_{n+1}$.

Let $r_{1}$ and $r_{n}$ be the geodesic rays in $\mathcal{P}_{n+1}$ defined as in (19). The ray $r_{i}, i=$ $1, n$, projects onto a maximal singular cusp ray in $\mathcal{T}_{n+1}$, which we denote by $\bar{r}_{i}$. The point at infinity $r_{1}(\infty)$ is the projective point $\left\langle e_{n+1}\right\rangle$. The point at infinity $r_{n}(\infty)$ is the hyperplane in $\mathbb{P}^{n} \mathbb{R}$ defined by $x_{1}=0$. We denote it by $e_{1}^{*}$.

The set $\mathcal{S}_{\psi}\left(\mathbb{R}^{n}\right)$ can be related to sets of geodesic rays similar to $\mathcal{R}_{\phi}^{i}$ from (37). The formula (1) will then imply a result similar to Theorem 5.1.2. More precisely, define

$$
\begin{aligned}
\mathcal{R}_{\phi}^{1} & =\left\{\mathbf{u} \in U_{+}\left(r_{1}\right) ;-f_{\bar{r}_{1}}\left(\operatorname{proj}\left(r_{1}(t) \mathbf{u}\right)\right)\right. \\
& \geq t-\phi(t) \text { infinitely many times as } t \rightarrow \infty\} .
\end{aligned}
$$

One can define a similar set for the ray $r_{n}$, that is

$$
\begin{aligned}
\mathcal{R}_{\phi}^{n} & =\left\{\mathbf{u} \in U_{+}\left(r_{n}\right) ;-f_{\bar{r}_{n}}\left(\operatorname{proj}\left(r_{n}(t) \mathbf{u}\right)\right)\right. \\
& \geq t-\phi(t) \text { infinitely many times as } t \rightarrow \infty\} .
\end{aligned}
$$

A remark similar to Remark 5.1.1 justifies the way $\mathcal{R}_{\phi}^{i}, i=1, n$, are defined. In the case when $t-\phi(t)=\beta t$ we replace in (42) and in (43) the index $\phi$ by $\beta$.

To simplify the formulas, we use the notation $\eta_{n}$ for the constant $\sqrt{\frac{n+1}{n}}$.

Theorem 5.2.1. Let $\phi:[a,+\infty) \rightarrow[b,+\infty)$ be a bijective function such that $\phi$ and $\eta_{n}^{2} i d-\phi$ are increasing functions. Then for $i=1, n$,

$$
\mathcal{H}^{s}\left(\mathcal{R}_{\phi}^{i}\right)= \begin{cases}0, & \text { if } \sum_{k=1}^{\infty} k^{n} e^{-\frac{s \eta_{n}}{2} \phi^{-1}\left(2 \eta_{n} \ln k\right)}<\infty, \\ \infty, & \text { if } \sum_{k=1}^{\infty} k^{n} e^{-\frac{s \eta_{n}}{2} \phi^{-1}\left(2 \eta_{n} \ln k\right)}=\infty\end{cases}
$$

Proof. Consider $\mathcal{S}_{\psi}^{e}\left(\mathbb{R}^{n}\right)$ the set defined as $\mathcal{S}_{\psi}\left(\mathbb{R}^{n}\right)$, but with the max-norm replaced by the Euclidean norm. We have that $\mathcal{S}_{\frac{1}{\sqrt{n}} \psi}\left(\mathbb{R}^{n}\right) \subset \mathcal{S}_{\psi}^{e}\left(\mathbb{R}^{n}\right) \subset \mathcal{S}_{\psi}\left(\mathbb{R}^{n}\right)$. This easily implies that (1) holds with $\mathcal{S}_{\psi}\left(\mathbb{R}^{n}\right)$ replaced by $\mathcal{S}_{\psi}^{e}\left(\mathbb{R}^{n}\right)$.

Step 1 . We first consider the set $\mathcal{R}_{\phi}^{1}$. We recall that according to (20),

$$
U_{+}\left(r_{1}\right)=\left\{\mathbf{u}_{\bar{x}}=\left(\begin{array}{cc}
I d_{n} & \bar{x} \\
0 & 1
\end{array}\right) ; \bar{x} \in \mathbb{R}^{n}\right\} .
$$

We may therefore identify $\mathbb{R}^{n}$ with $U_{+}\left(r_{1}\right)$ and thus identify $\mathcal{S}_{\psi}^{e}\left(\mathbb{R}^{n}\right)$ to a subset of $U_{+}\left(r_{1}\right)$, which we denote by $\widetilde{\mathcal{S}}_{\psi}$.

I. We prove that

$$
\mathcal{R}_{\phi}^{1} \subset \widetilde{\mathcal{S}}_{\psi_{1}}, \text { where } \psi_{1}(x)=x e^{-\frac{\eta_{n}}{2} \phi^{-1}\left(2 \eta_{n} \ln x\right)} .
$$


Let $\mathbf{u}_{\bar{x}} \in \mathcal{R}_{\phi}^{1}$. Infinitely many times as $t \rightarrow \infty$ we have that

$$
-f_{\bar{r}_{1}}\left(\operatorname{proj}\left(r_{1}(t) \mathbf{u}_{\bar{x}}\right)\right) \geq t-\phi(t) .
$$

By the discussion in the beginning of Section 3.6, this is equivalent to the statement that for infinitely many $t$ as $t \rightarrow \infty$ and infinitely many $(\bar{p}, q) \in \mathcal{P} \mathbb{Z}^{n+1}$,

$$
f_{(\bar{p}, q)}\left(r_{1}(t) \mathbf{u}_{\bar{x}}\right) \leq \phi(t)-t .
$$

The last inequality is equivalent to $r_{1}(t) \circ \mathbf{u}_{\bar{x}}(\bar{p}, q) \leq e^{\frac{\phi(t)-t}{\eta_{n}}}$, which in its turn writes as

$$
e^{\frac{t}{\sqrt{n(n+1)}}}\|\bar{p}+q \bar{x}\|_{e}^{2}+e^{-\frac{n t}{\sqrt{n(n+1)}}} q^{2} \leq e^{\frac{\phi(t)-t}{\eta_{n}}} .
$$

The inequality $e^{-\frac{n t}{\sqrt{n(n+1)}}} q^{2} \leq e^{\frac{\phi(t)-t}{\eta_{n}}}$ is equivalent by monotonicity of $\phi$ with

$$
t \geq \phi^{-1}\left(2 \eta_{n} \ln q\right) \text {. }
$$

The inequality $e^{\frac{t}{\sqrt{n(n+1)}}}\|\bar{p}+q \bar{x}\|_{e}^{2} \leq e^{\frac{\phi(t)-t}{\eta_{n}}}$ then implies that $\|\bar{p}+q \bar{x}\|_{e}^{2} \leq$ $e^{\frac{\phi(t)}{\eta_{n}}-\eta_{n} t}$. This, inequality (47) and the fact that the function $\eta_{n}^{2} i d-\phi$ is increasing, imply that

$$
\|\bar{p}+q \bar{x}\|_{e} \leq \psi_{1}(q)
$$

II. We prove that

$$
\widetilde{\mathcal{S}}_{\psi_{2}} \subset \mathcal{R}_{\phi}^{1}, \text { where } \psi_{2}(x)=x e^{-\frac{\eta_{n}}{2} \phi^{-1}\left(2 \eta_{n} \ln (\sqrt{2} x)\right)} .
$$

Let $\mathbf{u}_{\bar{x}} \in \widetilde{\mathcal{S}}_{\psi_{2}}$. Then for infinitely many $(\bar{p}, q) \in \mathcal{P} \mathbb{Z}^{n+1}$,

$$
\|\bar{p}+q \bar{x}\|_{e} \leq \psi_{2}(q) .
$$

We take $t=\phi^{-1}\left(2 \eta_{n} \ln (\sqrt{2} q)\right)$ and consider

$$
e^{\frac{t}{\sqrt{n(n+1)}}}\|\bar{p}+q \bar{x}\|_{e}^{2}+e^{-\frac{n t}{\sqrt{n(n+1)}}} q^{2} .
$$

By the choice of $t$, the second term of the sum is equal to $\frac{1}{2} e^{\frac{\phi(t)-t}{\eta_{n}}}$. The first term is at most

$$
e^{\frac{t}{\sqrt{n(n+1)}}} \psi_{2}(q)^{2}=e^{\frac{t}{\sqrt{n(n+1)}}} q^{2} e^{-\eta_{n} t}=\frac{1}{2} e^{\frac{\phi(t)-t}{\eta_{n}}} .
$$

We conclude that

$$
e^{\frac{t}{\sqrt{n(n+1)}}}\|\bar{p}+q \bar{x}\|_{e}^{2}+e^{-\frac{n t}{\sqrt{n(n+1)}}} q^{2} \leq e^{\frac{\phi(t)-t}{\eta n}}
$$

This implies inequality (46) for the chosen $t=t(q)$. Hence inequality (46) is satisfied infinitely many times as $t \rightarrow \infty$, consequently $\mathbf{u}_{\bar{x}} \in \mathcal{R}_{\phi}$.

The double inclusion $\widetilde{\mathcal{S}}_{\psi_{2}} \subset \mathcal{R}_{\phi}^{1} \subset \widetilde{\mathcal{S}}_{\psi_{1}}$ and (1) with $\mathcal{S}_{\psi}\left(\mathbb{R}^{n}\right)$ replaced by $\mathcal{S}_{\psi}^{e}\left(\mathbb{R}^{n}\right)$ imply the conclusion. Note that in the divergence part, what appears is the sum in (1) for the function $\psi_{2}$. The function $\psi_{2}$ differs from $\psi_{1}$ by a factor $\sqrt{2}$ in front of the variable, in the argument of $\ln$. Nevertheless, it is easy to eliminate this factor from the sum with an argument as in the proof of Remark 5.2.2. 
Step 2. We now consider the set $\mathcal{R}_{\phi}^{n}$. By (20),

$$
U_{+}\left(r_{n}\right)=\left\{\mathbf{u}_{\bar{x}}=\left(\begin{array}{cc}
1 & \bar{x}^{T} \\
0 & I d_{n}
\end{array}\right) ; \bar{x} \in \mathbb{R}^{n}\right\} .
$$

We identify $\mathbb{R}^{n}$ with $U_{+}\left(r_{n}\right)$ and thereby $\mathcal{S}_{\psi}^{e}\left(\mathbb{R}^{n}\right)$ to a subset of $U_{+}\left(r_{n}\right)$, denoted by $\overline{\mathcal{S}}_{\psi}$.

The pre-image of $H b_{a}\left(\bar{r}_{n}\right)$ is $\bigcup_{v \in \mathcal{P} \mathbb{Z}^{n+1}} H b_{v^{*}}^{a}$. Also, for every $v=(q, \bar{p}) \in$ $\mathcal{P} \mathbb{Z}^{n+1}$,

$$
\begin{aligned}
f_{v}^{*}\left(r_{n}(t) \mathbf{u}_{\bar{x}}\right) & =\eta_{n} \ln \left[\left(r_{n}(t) \mathbf{u}_{\bar{x}}\right)^{*}(q, \bar{p})\right] \\
& =\eta_{n} \ln \left(e^{-\frac{n t}{\sqrt{n(n+1)}}} q^{2}+e^{\frac{t}{\sqrt{n(n+1)}}}\|\bar{p}-q \bar{x}\|_{e}^{2}\right) .
\end{aligned}
$$

An argument almost identical to the one of Step 1 gives that

$$
\overline{\mathcal{S}}_{\psi_{2}} \subset \mathcal{R}_{\phi}^{n} \subset \overline{\mathcal{S}}_{\psi_{1}} .
$$

This together with (1) implies the conclusion.

Remark 5.2.2. In the definition of the set $\mathcal{R}_{\phi}^{i}$, one can replace $\phi$ by $\phi_{c}=\phi-c$, where $c$ is a constant, and the conclusion of Theorem 5.2.1 still holds.

Proof. Without loss of generality we may assume that $c>0$ (the case $c<$ 0 is obtained by intertwining $\phi$ with $\phi_{c}$ ). Theorem 5.2.1 applied to the function $\phi_{c}$ gives (44) with $\phi_{c}$ instead of $\phi$. The sum appearing in (44) is $\Sigma_{c}=$ $\sum_{k=1}^{\infty} k^{n} e^{-\frac{s \eta_{n} \phi^{-1}\left(2 \eta_{n} \ln k+c\right)}{2}} \leq \Sigma_{0}=\sum_{k=1}^{\infty} k^{n} e^{-\frac{s \eta_{n} \phi^{-1}\left(2 \eta_{n} \ln k\right)}{2}}$. If $\Sigma_{0}<\infty$ then $\Sigma_{c}<\infty$.

Suppose that $\Sigma_{0}=\infty$. Let $p>0$ such that $e^{\frac{c}{2 \eta_{n}}} \leq 2^{p}$. There exists $r \in$ $\left\{0,1, \ldots, 2^{p}-1\right\}$ such that $\Sigma_{0}(r)=\sum_{k \in 2^{p} \mathbb{Z}+r} k^{n} e^{-\frac{s \eta_{n} \phi^{-1}\left(2 \eta_{n} \ln k\right)}{2}}=\infty$. On the other hand

$$
\Sigma_{c} \geq \sum_{k=1}^{\infty} k^{n} e^{-\frac{s \eta_{n} \phi^{-1}\left(2 \eta_{n} \ln \left(2^{p} k+r\right)\right)}{2}},
$$

and the latter sum is $+\infty$ because $\Sigma_{0}(r)=+\infty$.

Corollary 5.2.3. (i) For any $\beta \in(0,1)$, the set $\mathcal{R}_{\beta}^{i}, i=1, n$, has Hausdorff dimension $d=n(1-\beta)$ and $\mathcal{H}^{d}\left(\mathcal{R}_{\beta}^{i}\right)=\infty$.

(ii) Both statements remain true for the set

$$
\mathcal{E R}_{\beta}^{i}=\mathcal{R}_{\beta}^{i} \backslash \bigcup_{\beta^{\prime}>\beta} \mathcal{R}_{\beta^{\prime}}^{i}=\left\{\mathbf{u} \in \mathcal{R}_{\beta}^{i} ; \limsup _{t \rightarrow \infty} \frac{-f_{\bar{r}_{i}}\left(\operatorname{proj}\left(r_{i}(t) \mathbf{u}\right)\right)}{t}=\beta\right\} .
$$

(iii) The set $\mathcal{R}_{0}^{i}$ coincides with $U_{+}\left(r_{i}\right)$. The set $\mathcal{R}_{1}^{1}$ is contained in $\left\{\mathbf{u} \in U_{+}\left(r_{1}\right)\right.$; $\left.r_{1} \mathbf{u}(\infty) \in \mathcal{P} \mathbb{Z}^{n+1}\right\}$ and the set $\mathcal{R}_{1}^{n}$ is contained in $\left\{\mathbf{u} \in U_{+}\left(r_{n}\right) ; r_{n} \mathbf{u}(\infty) \in\right.$ $\left.\left(\mathcal{P} \mathbb{Z}^{n+1}\right)^{*}\right\}$.

Proof. (i) follows from Theorem 5.2.1, (ii) follows from (i), (iii) is obtained as Corollary 5.1.5, (c). 


\subsection{Case when the ray measuring the depth has a different slope}

We now try to relate the set $\mathcal{L}_{\psi}\left(\mathbb{R}^{n}\right)$ to sets of geodesic rays similar to $\mathcal{R}_{\phi}^{i}, i=1, n$, from (42) and (43), and to reformulate (4) in terms of their Hausdorff measure. It turns out that the sets $\mathcal{R}_{\phi}$ to be considered in this case are a bit different from all the sets considered before. More precisely, what has to be considered is either the set of rays of slope $r_{1}(\infty)$ and their divergence measured with respect to $\bar{r}_{n}$ or the set of rays of slope $r_{n}(\infty)$ and their divergence measured with respect to $\bar{r}_{1}$. Before defining them, we remark that the maximal possible depth of proj $\left(r_{i}(t) \mathbf{u}\right)$ measured with respect to $\bar{r}_{j}$, when $\{i, j\}=\{1, n\}$, is $\frac{1}{n} t+c$, where $c$ is a constant. Such a depth occurs infinitely many times if and only if the ray $\operatorname{proj}\left(r_{i} \mathbf{u}\right)$ is contained in the projection of a Weyl chamber with a 1-dimensional face asymptotic to $\bar{r}_{j}$ (see Corollary 5.3.4, (iii)). Hence in this case in the definitions of the sets of rays moving away in the cusp one must put $\frac{1}{n} t-\phi(t)$ with $\phi:[a,+\infty) \rightarrow[b,+\infty)$.

Thus we define

$$
\begin{aligned}
\mathcal{R}_{\phi}^{1 n}=\{\mathbf{u} & \in U_{+}\left(r_{1}\right) ;-f_{\bar{r}_{n}}\left(\operatorname{proj}\left(r_{1}(t) \mathbf{u}\right)\right) \\
\geq & \left.\frac{1}{n} t-\phi(t) \text { infinitely many times as } t \rightarrow \infty\right\} .
\end{aligned}
$$

Similarly we define

$$
\begin{aligned}
\mathcal{R}_{\phi}^{n 1}=\{\mathbf{u} & \in U_{+}\left(r_{n}\right) ;-f_{\bar{r}_{1}}\left(\operatorname{proj}\left(r_{n}(t) \mathbf{u}\right)\right) \\
\geq & \left.\frac{1}{n} t-\phi(t) \text { infinitely many times as } t \rightarrow \infty\right\} .
\end{aligned}
$$

Let $\mathcal{L}_{\psi}^{e}\left(\mathbb{R}^{n}\right)$ be the set defined as $\mathcal{L}_{\psi}\left(\mathbb{R}^{n}\right)$ but with the max-norm replaced by the Euclidean norm. We need to replace in $(4) \mathcal{L}_{\psi}\left(\mathbb{R}^{n}\right)$ by $\mathcal{L}_{\psi}^{e}\left(\mathbb{R}^{n}\right)$.

Lemma 5.3.1. Let $\psi$ be an approximating function such that $\psi_{1}(x)=\frac{\psi(x)}{x}$ is a decreasing function. Then (4) holds with $\mathcal{L}_{\psi}\left(\mathbb{R}^{n}\right)$ replaced by $\mathcal{L}_{\psi}^{e}\left(\mathbb{R}^{n}\right)$.

Proof. The hypothesis on $\psi$ implies that

$$
\mathcal{L}_{\frac{1}{\sqrt{n}} \psi(\sqrt{n} \cdot)}\left(\mathbb{R}^{n}\right) \subset \mathcal{L}_{\psi}^{e}\left(\mathbb{R}^{n}\right) \subset \mathcal{L}_{\sqrt{n} \psi}\left(\mathbb{R}^{n}\right) .
$$

This and (4) yield

$$
\mathcal{H}^{s}\left(\mathcal{L}_{\psi}^{e}\left(\mathbb{R}^{n}\right)\right)= \begin{cases}0, & \text { if } \sum_{k=1}^{\infty} k^{n} \psi_{1}(k)^{s-(n-1)}<\infty, \\ \infty, & \text { if } \sum_{k=1}^{\infty} k^{n} \psi_{1}(\sqrt{n} k)^{s-(n-1)}=\infty\end{cases}
$$

Let $p \in \mathbb{N}$ be such that $2^{p} \geq \sqrt{n}$. The hypothesis $\sum_{k=1}^{\infty} k^{n} \psi_{1}(k)^{s-(n-1)}=\infty$ implies that there exists $r \in\left\{0,1, \ldots, 2^{p}-1\right\}$ such that $\sum_{k \in 2^{p} \mathbb{Z}+r} k^{n} \psi_{1}(k)^{s-(n-1)}$ 
$=\infty$. We have that $\sum_{k=1}^{\infty} k^{n} \psi_{1}(\sqrt{n} k)^{s-(n-1)} \geq \sum_{k=1}^{\infty} k^{n} \psi_{1}\left(2^{p} k+r\right)^{s-(n-1)}$, which implies that

$$
\sum_{k=1}^{\infty} k^{n} \psi_{1}(\sqrt{n} k)^{s-(n-1)}=\infty
$$

Hence (49) can be re-written such that the sum $\sum_{k=1}^{\infty} k^{n} \psi_{1}(k)^{s-(n-1)}$ also appears on the second line.

Theorem 5.3.2. Let $\phi:[a,+\infty) \rightarrow[b,+\infty)$ be a bijective function such that $\phi$ and $\eta_{n}^{2} i d-\phi$ are increasing functions. Then for $\{i, j\}=\{1, n\}$,

$$
\mathcal{H}^{s}\left(\mathcal{R}_{\phi}^{i j}\right)= \begin{cases}0, & \text { if } \sum_{k=1}^{\infty} k^{n} e^{-(s-n+1) \frac{\eta_{n}}{2} \phi^{-1}\left(2 \eta_{n} \ln k\right)}<\infty, \\ \infty, & \text { if } \sum_{k=1}^{\infty} k^{n} e^{-(s-n+1) \frac{\eta_{n}}{2} \phi^{-1}\left(2 \eta_{n} \ln k\right)}=\infty .\end{cases}
$$

Proof. We give a proof only for $i=1, j=n$, the argument in the other case is similar. As in Step 1 of the proof of Theorem 5.2.1, we can identify $\mathbb{R}^{n}$ to $U_{+}\left(r_{1}\right)$ and thus identify $\mathcal{L}_{\psi}^{e}\left(\mathbb{R}^{n}\right)$ to a subset of $U_{+}\left(r_{1}\right)$, which we denote by $\widetilde{\mathcal{L}}_{\psi}$. We prove that

$$
\widetilde{\mathcal{L}}_{\psi_{2}} \subset \mathcal{R}_{\phi}^{1 n} \subset \widetilde{\mathcal{L}}_{\psi_{1}},
$$

where $\psi_{1}$ and $\psi_{2}$ are the functions defined in (45) and (48), respectively.

I. We prove the second inclusion in (50). Let $\mathbf{u}_{\bar{x}} \in \mathcal{R}_{\phi}^{1 n}$. Then for infinitely many $t>0$ and $v=(\bar{p}, q) \in \mathcal{P} \mathbb{Z}^{n+1}$ we have that

$$
f_{v}^{*}\left(r_{1}(t) \mathbf{u}_{\bar{x}}\right) \leq \phi(t)-\frac{1}{n} t \Leftrightarrow \eta_{n} \ln \left[\left(r_{1}(t) \mathbf{u}_{\bar{x}}\right)^{*}(\bar{p}, q)\right] \leq \phi(t)-\frac{1}{n} t .
$$

The inequality above is equivalent to

$$
e^{-\frac{t}{\sqrt{n(n+1)}}}\|\bar{p}\|_{e}^{2}+e^{\frac{n t}{\sqrt{n(n+1)}}}(\bar{p} \cdot \bar{x}-q)^{2} \leq e^{\frac{\phi(t)}{\eta_{n}}-\frac{t}{\sqrt{n(n+1)}}} .
$$

The inequality $e^{-\frac{t}{\sqrt{n(n+1)}}}\|\bar{p}\|_{e}^{2} \leq e^{\frac{\phi(t)}{\eta_{n}}-\frac{t}{\sqrt{n(n+1)}}}$ is equivalent to

$$
t \geq \phi^{-1}\left(2 \eta_{n} \ln \|\bar{p}\|_{e}\right) \text {. }
$$

The inequality $e^{\frac{n t}{\sqrt{n(n+1)}}}(\bar{p} \cdot \bar{x}-q)^{2} \leq e^{\frac{\phi(t)}{\eta n}-\frac{t}{\sqrt{n(n+1)}}}$ implies that

$$
(\bar{p} \cdot \bar{x}-q)^{2} \leq e^{\frac{\phi(t)}{\eta_{n}}-\eta_{n} t} .
$$

The hypothesis that $\eta_{n}^{2} i d-\phi$ is an increasing function, together with (51) and (52) imply that $|\bar{p} \cdot \bar{x}-q| \leq \psi_{1}\left(\|\bar{p}\|_{e}\right)$. 
II. We prove the first inclusion in (50). Let $\mathbf{u}_{\bar{x}} \in \widetilde{\mathcal{L}}_{\psi_{2}}$. It follows that for infinitely many $(\bar{p}, q) \in \mathcal{P} \mathbb{Z}^{n+1}$, we have $|\bar{p} \cdot \bar{x}-q| \leq \psi_{2}\left(\|\bar{p}\|_{e}\right)$. Consider $t=$ $\phi^{-1}\left(2 \eta_{n} \ln \left(\sqrt{2}\|\bar{p}\|_{e}\right)\right)$ and the expression

$$
e^{-\frac{t}{\sqrt{n(n+1)}}}\|\bar{p}\|_{e}^{2}+e^{\frac{n t}{\sqrt{n(n+1)}}}(\bar{p} \cdot \bar{x}-q)^{2} .
$$

The choice of $t$ implies that $e^{-\frac{t}{\sqrt{n(n+1)}}}\|\bar{p}\|_{e}^{2}=\frac{1}{2} e^{\frac{\phi(t)}{\eta_{n}}-\frac{t}{\sqrt{n(n+1)}}}$. We also have

$$
e^{\frac{n t}{\sqrt{n(n+1)}}}(\bar{p} \cdot \bar{x}-q)^{2} \leq e^{\frac{n t}{\sqrt{n(n+1)}}} \psi_{2}\left(\|\bar{p}\|_{e}\right)^{2}=\frac{1}{2} e^{\frac{\phi(t)}{\eta n}-\frac{t}{\sqrt{n(n+1)}}} .
$$

Thus the expression in (53) is at most $e^{\frac{\phi(t)}{\eta_{n}}-\frac{t}{\sqrt{n(n+1)}}}$, which implies that

$$
f_{(\bar{p}, q)}^{*}\left(r_{1}(t) \mathbf{u}_{\bar{x}}\right) \leq \phi(t)-\frac{1}{n} t .
$$

Since this holds for infinitely many $(\bar{p}, q) \in \mathcal{P} \mathbb{Z}^{n+1}$, we obtain that $\mathbf{u}_{\bar{x}} \in \mathcal{R}_{\phi}^{1 n}$. The double inclusion (50) and Lemma 5.3.1 yield the conclusion.

Remark 5.3.3. In the definition of the sets $\mathcal{R}_{\phi}^{i j}$, the function $\phi$ can be replaced by $\phi_{c}=\phi-c$, where $c$ is a constant, and Theorem 5.3.2 still holds.

Proof. Similar to the one of Remark 5.2.2.

Corollary 5.3.4. (i) For any $\beta \in\left(0, \frac{1}{n}\right)$, the set $\mathcal{R}_{\beta}^{i j},\{i, j\}=\{1, n\}$, has Hausdorff dimension $d=n(1-\beta)$ and $\mathcal{H}^{d}\left(\mathcal{R}_{\beta}^{i j}\right)=\infty$.

(ii) Both statements remain true for the set

$$
\mathcal{E} \mathcal{R}_{\beta}^{i j}=\mathcal{R}_{\beta}^{i j} \backslash \bigcup_{\beta^{\prime}>\beta} \mathcal{R}_{\beta^{\prime}}^{i j}=\left\{\mathbf{u} \in \mathcal{R}_{\beta}^{i j} ; \limsup _{t \rightarrow \infty} \frac{-f_{\bar{r}_{j}}\left(\operatorname{proj}\left(r_{i}(t) \mathbf{u}\right)\right)}{t}=\beta\right\} .
$$

(iii) The set $\mathcal{R}_{0}^{i j}$ coincides with $U_{+}\left(r_{i}\right)$.

The set $\mathcal{R}_{\frac{1}{n}}^{1 n}$ is a subset of the set of $\mathbf{u} \in U_{+}\left(r_{1}\right)$ such that the projective point $r_{1} \mathbf{u}(\infty)$ is contained in one of the hyperplanes of equation $x_{i}=q$, where $q \in \mathbb{Z}$ and $i \in\{1,2, \ldots, n\}$.

The set $\mathcal{R}_{\frac{1}{n}}^{n 1}$ is a subset of the set of $\mathbf{u} \in U_{+}\left(r_{n}\right)$ such that the hyperplane $r_{n} \mathbf{u}(\infty)$ contains one of the vectors $e_{i}+q e_{n+1}$, where $q \in \mathbb{Z}$ and $i \in$ $\{1,2, \ldots, n\}$.

Proof. (i) follows from Theorem 5.3.2 and (ii) follows from (i).

The first statement in (iii) is again a consequence of the logarithm law $\left[\mathrm{KM}_{1}\right]$.

We prove the second statement. The proof of the third is similar. 
Let $\mathbf{u}_{\bar{x}} \in \mathcal{R}_{\frac{1}{n}}^{1 n}$. As in the proof of Theorem 5.3.2, I, we deduce that there exist infinitely many $(\bar{p}, q) \in \mathcal{P} \mathbb{Z}^{n+1}$ and $t>0$ such that

$$
e^{-\frac{t}{\sqrt{n(n+1)}}}\|\bar{p}\|_{e}^{2}+e^{\frac{n t}{\sqrt{n(n+1)}}}(\bar{p} \cdot \bar{x}-q)^{2} \leq e^{-\frac{t}{\sqrt{n(n+1)}}} .
$$

It follows that $\|\bar{p}\|_{e}^{2} \leq 1$ and that $(\bar{p} \cdot \bar{x}-q)^{2} \leq e^{-\eta_{n} t}$. The first inequality implies that $\bar{p}=e_{i} \in \mathbb{R}^{n}$ for some $i \in\{1,2, \ldots, n\}$. We may suppose that for infinitely many $t>0$ it is the same $i$. The second inequality gives $\left(x_{i}-q\right)^{2} \leq e^{-\eta_{n} t}$, for infinitely many $t$, as $t \rightarrow \infty$. There are finitely many possibilities for $q$, so again we may suppose that in the previous sequence of inequalities $q$ is fixed. Then as $t \rightarrow \infty$, this gives $x_{i}=q$.

Acknowledgements. I am grateful to the referee for pointing out to me the references [BDV $\left.{ }_{1}\right]$ and $\left[\mathrm{BDV}_{2}\right]$, which allowed me to improve the results that I had in the first draft of this paper. I wish to thank Livio Flaminio for having explained to me the equidistribution result Proposition 2.6.5 in the case of $S L(2, \mathbb{R})$ as well as for many useful conversations. I also thank Maurice Dodson for inspiring conversations and for providing useful references.

\section{References}

[Bak] Baker, R.C.: Dirichlet's theorem on Diophantine approximation. Math. Proc. Cambridge Philos. Soc. 83, 37-59 (1978)

[BaS] Baker, A., Schmidt, W.M.: Diophantine approximation and Hausdorff dimension. Proc. London Math. Soc. 21, 1-11 (1970)

[BGS] Ballmann, W., Gromov, M., Schroeder, V.: Manifolds of nonpositive curvature, Birkhäuser, 1985

[Ber] Beresnevich, V.V.: A Groshev type theorem for convergence on manifolds. Acta Math. Hungarica 94, 99-130 (2002)

[BBKM] Beresnevich, V.V., Bernik, V.I., Kleinbock, D., Margulis, G.A.: Metric Diophantine Approximation: the Khintchine-Groshev Theorem for non-degenerated manifolds. Moscow Math. J. 2, 203-225 (2002)

$\left[\mathrm{BDV}_{1}\right]$ Beresnevich, V.V., Dickinson, H., Velani, S.L.: Diophantine approximation on planar curves and the distribution of rational points. preprint arXiv:math.NT/0401148

$\left[\mathrm{BDV}_{2}\right]$ Beresnevich, V.V., Dickinson, H., Velani, S.L.: Measure theoretic laws for lim sup sets. preprint arXiv:math.NT/0401118

$\left[\mathrm{BDV}_{3}\right]$ Beresnevich, V.V., Dickinson, H., Velani, S.L.: Sets of exact 'logarithmic' order in the theory of Diophantine approximation. Math. Ann. 321, 253-273 (2001)

[Be] Berger, M.: Géométrie, vol. 4 ("Formes quadratiques, quadriques et coniques"), Cedic/Fernand Nathan, 1978

[BD] Bernik, V.I., Dodson, M.M.: Metric Diophantine Approximation on Manifolds. Cambridge Univ. Press, 1999

[BKM] Bernik, V.I., Kleinbock, D., Margulis, G.A.: Khintchine-type theorems on manifolds: the convergence case for standard and multiplicative versions. IMRN 9, 453-486 (2001)

[BoD] Bovey, J.D., Dodson, M.M.: The Hausdorff dimension of systems of linear forms. Acta Arithm. 45, 337-358 (1986) 
[BoS] Borel, A., Serre, J.-P.: Corners and arithmetic groups. Avec un appendice: Arrondissement des variétés à coins. par A. Douady et L. Hérault, Comment. Math. Helv. 48, 436-491 (1973)

[Bo] Borel, A.: Introduction aux groupes arithmétiques. Hermann, Paris, 1969

[BSh] Borevich, Z.I., Shafarevich, I.R.: Théorie des nombres. Gauthier-Villars, Paris, 1967

[BH] Bridson, M.R., Haefliger, A.: Metric Spaces of Nonpositive Curvature. Birkhäuser, 1985

[Bu] Bugeaud, Y.: An inhomogeneous Jarník theorem. Preprint 2003

[DD $D_{1}$ Dickinson, H., Dodson, M.M.: Simultaneous Diophantine Approximation on the circle and Hausdorff dimension. Math. Proc. Cambridge Philos. Soc. 130, 515-522 (2001)

[DD $\left.\mathrm{DD}_{2}\right]$ Dickinson, H., Dodson, M.M.: Extremal manifolds and Hausdorff dimension. Duke Math. J. 101, 271-281 (2000)

[DL] Dickinson, H., Levesly, J.: Simultaneous Diophantine approximation on polynomial surfaces. Preprint

[DV] Dickinson, H., Velani, S.L.: Hausdorff measure and linear forms. J. reine angew. Math. 490, 1-36 (1997)

$\left[\mathrm{DRV}_{1}\right]$ Dodson, M.M., Rynne, B.P., Vickers, J.A.G.: Metric Diophantine approximation and Hausdorff dimension on manifolds. Math. Proc. Cambridge Philos. Soc. 105, 547558 (1989)

$\left[\mathrm{DRV}_{2}\right]$ Dodson, M.M., Rynne, B.P., Vickers, J.A.G.: Diophantine approximation and a lower bound for Hausdorff dimension. Mathematika 37, 59-73 (1990)

[DRV 3 ] Dodson, M.M., Rynne, B.P., Vickers, J.A.G.: Khintchine-type theorems on manifolds. Acta Arith. 57, 115-130 (1991)

[DRV 4 Dodson, M.M., Rynne, B.P., Vickers, J.A.G.: Simultaneous Diophantine approximation and asymptotic formulae on manifolds. J. Number Theory 58, 298-316 (1996)

[EMM] Eskin, A., Margulis, G.A., Mozes, S.: Upper bounds and asymptotics in a quantitative version of the Oppenheim conjecture. Ann. Math. 147, 93-141 (1998)

[He] Helgason, S.: Differential Geometry, Lie Groups and Symmetric Spaces. Academic Press, 1978

[HV] Hill, R., Velani, S.L.: The Jarník-Besicovitch Theorem for geometrically finite kleinian groups. Proc. London Math. Soc. 77, 524-550 (1998)

[Ja] Jarník, V.: Über die simultanen diophantischen Approximationen. Math. Z. 33, 503 $543(1931)$

$\left[\mathrm{KM}_{1}\right] \quad$ Kleinbock, D., Margulis, G.A.: Logarithm laws for flows on homogeneous spaces. Invent. math. 138, 451-494 (1999)

$\left[\mathrm{KM}_{2}\right] \quad$ Kleinbock, D., Margulis, G.A.: Flows on homogeneous spaces and Diophantine approximation on manifolds. Ann. Math. 148, 339-360 (1998)

[Le] Leuzinger, E.: An exhaustion of locally symmetric spaces by compact submanifolds with corners. Invent. Math. 121, 389-410 (1995)

[Mo] Mostow, G.D.: Strong rigidity of locally symmetric spaces. Ann. Math. Studies No. 78, Princeton University Press, 1973

[OV] Onishchik, A., Vinberg, E.: Lie Groups and Algebraic Groups. Springer Verlag, 1990

[Pa] Paterson, A.L.T.: Amenability. Math. Surveys and Monographs no. 29, AMS, 1988

[Ra] Raghunathan, M.S.: Discrete subgroups of Lie groups. Springer Verlag, 1972

[Ry] Rynne, B.P.: Simultaneous Diophantine approximation on manifolds and Hausdorff dimension. J. Number Theory 98, 1-9 (2003)

[Su] Sullivan, D.: Disjoint spheres, approximation by imaginary quadratic numbers, and the logarithm law for geodesics. Acta Math. 149, 215-237 (1982)

[Wi] Witte Morris, D.: Introduction to Arithmetic groups. http://www.math. okstate.edu/ $\sim$ dwitte

[Zi] Zimmer, R.J.: Ergodic theory and semisimple groups. Monographs in Mathematics, 81, Birkhäuser Verlag, Basel, 1984 